\title{
Disease-Gene Association Using a Genetic Algorithm
}

\author{
Koosha Tahmasebipour \\ Department of Computer Science
}

Submitted in partial fulfillment

of the requirements for the degree of

Master of Science

Faculty of Mathematics and Science, Brock University St. Catharines, Ontario

(C)August, 2014 


\section{Abstract}

Understanding the relationship between genetic diseases and the genes associated with them is an important problem regarding human health. The vast amount of data created from a large number of high-throughput experiments performed in the last few years has resulted in an unprecedented growth in computational methods to tackle the disease gene association problem. Nowadays, it is clear that a genetic disease is not a consequence of a defect in a single gene. Instead, the disease phenotype is a reflection of various genetic components interacting in a complex network. In fact, genetic diseases, like any other phenotype, occur as a result of various genes working in sync with each other in a single or several biological module(s). Using a genetic algorithm, our method tries to evolve communities containing the set of potential disease genes likely to be involved in a given genetic disease. Having a set of known disease genes, we first obtain a protein-protein interaction (PPI) network containing all the known disease genes. All the other genes inside the procured PPI network are then considered as candidate disease genes as they lie in the vicinity of the known disease genes in the network. Our method attempts to find communities of potential disease genes strongly working with one another and with the set of known disease genes. As a proof of concept, we tested our approach on 16 breast cancer genes and 15 Parkinson's Disease genes. We obtained comparable or better results than CIPHER, ENDEAVOUR and GPEC, three of the most reliable and frequently used disease-gene ranking frameworks. 


\section{Acknowledgements}

I would like to express my gratitude to everyone who supported me throughout my M.Sc. research:

- I express my deep gratitude to my supervisor, Professor Sheridan Houghten, for her inspiring guidance, financial support, and all her spectacular advice during this research.

- I extend my warm thanks to Cale Fairchild for all his technical support.

- My heartfelt thanks to my parents, without whom I would never have been in this stage.

- And my grateful thanks to Kimia, for her special care and concern to me to do this well and on time.

K.T 


\section{Contents}

1 Introduction 1

1.1 Thesis Structure . . . . . . . . . . . . . . . 2

2 Background 3

2.1 Bioinformatics: promises and applications . . . . . . . . . . 3

2.2 Basic Biological Concepts . . . . . . . . . . . . . . 4

2.2.1 Genes, the living organisms' algorithms and software . . . . . 4

2.2.2 The living organisms hardware: the proteins . . . . . . . . 5

2.2.3 Genetic disease as a type of phenotype . . . . . . . . . . 6

2.3 Disease-Gene Association Problem . . . . . . . . . . . . . 8

2.3.1 Bioinformatics and Computational Disease-Gene Association . 9

2.3.2 Known Disease Genes and the Principle of Guilt by Association 10

2.3.3 Disease-Gene Association as a Complex Problem . . . . . . . 10

3 Literature Review of Disease-Gene Association 12

3.1 Definition of Computational Disease-Gene Association . . . . . . . . 12

3.2 Classification of Existing Evidence for Disease-Gene Prediction in the Literature . . . . . . . . . . . . . . . . . . 13

3.2.1 Text Mining of the Literature . . . . . . . . . . . . . . 13

3.2.2 Functional Evidence and Pathways . . . . . . . . . . . . . 14

3.2.3 Phenotypic Evidence . . . . . . . . . . . . . . . 14

3.2.4 Inherent Gene Properties . . . . . . . . . . . . . . . 15

3.2.5 Sequencing Data and Mutation Evidence . . . . . . . . . . 15

3.2.6 Protein-Protein Interaction . . . . . . . . . . . . . . . . 15

3.2.7 Gene Expression Data . . . . . . . . . . . . . . 16

3.3 Applying a Fusion of Different Evidence and Resources . . . . . . . . 17 
4 Review of using PPI for Disease-Gene Association 18

4.1 Neighbourhood-based Algorithms . . . . . . . . . . . . . . . 18

4.2 Random Walk Algorithms . . . . . . . . . . . . . . . . . . . . 19

4.3 Graph Partitioning Algorithms . . . . . . . . . . . . . . . 19

5 Methodologies $\quad 20$

5.1 Science of Complexity . . . . . . . . . . . . . . . . . 20

5.1.1 Complex Networks . . . . . . . . . . . . . . 21

5.1.2 Community Identification Problem in Complex Networks . . . 21

5.1.3 Disease-Gene Association as a Community Identification Problem 23

5.2 What is a Genetic Algorithm? . . . . . . . . . . . . . . . 24

6 The Design of Our Computational Approach 26

6.1 General Idea . . . . . . . . . . . . . . . . . . . . . 26

6.2 Motivations and Ideas . . . . . . . . . . . . . . . . . 27

6.2.1 Why use a Community Identification Approach? . . . . . . . . 27

6.2.2 Why use a GA for Community Identification? . . . . . . . . . 27

6.3 Details of the Genetic Algorithm . . . . . . . . . . . . . . . 28

6.3.1 Fitness Evaluation . . . . . . . . . . . . . 28

6.3.2 Individuals (Chromosomes) . . . . . . . . . . . . . 29

6.3.3 Linkage with known disease genes: Guilt by Association . . . 30

6.3.4 Maximum Community Size . . . . . . . . . . . . . . . . 30

6.4 Prioritization of the Candidate Genes . . . . . . . . . . . . . . . . . 31

6.4.1 Scoring System . . . . . . . . . . . . . . . 31

7 Datasets and Benchmark Tests 33

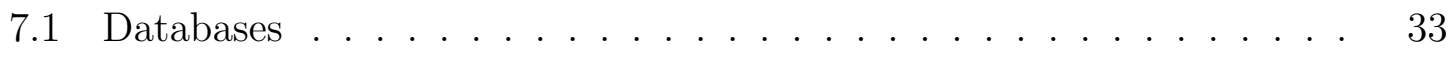

7.1.1 Protein-Protein Interaction Data . . . . . . . . . . . . . 33

7.1 .2 Disease-Gene Association Data . . . . . . . . . . . . . . . 33

7.2 Benchmark Test . . . . . . . . . . . . . . . . . . 34

7.2.1 Leave-One-Out Cross-Validation . . . . . . . . . . . . . . . . 34

7.2.2 Fold enrichment analysis . . . . . . . . . . . . . . . 34

7.2.3 Receiver-Operating Characteristic (ROC) analysis . . . . . . . 34

8 Case Studies and Results 36

8.1 Breast Cancer . . . . . . . . . . . . . . . . . 36

8.1 .1 Input Data . . . . . . . . . . . . . 36 
8.1 .2 GA Parameters . . . . . . . . . . . . . . . 36

8.2 Analysis . . . . . . . . . . . . . . . . . . . . . . 38

8.2.1 Leave-One-Out Cross-Validation . . . . . . . . . . . . . . 38

8.2 .2 Comparison with CIPHER . . . . . . . . . . . . . . . 38

8.2 .3 Comparison with Random Walk . . . . . . . . . . . . . . 39

8.2.4 Summary of the Analysis for Breast Cancer Experiment . . . 40

8.2.5 Predicting new Disease Genes . . . . . . . . . . . . . . . 40

8.3 Parkinson's Disease . . . . . . . . . . . . . . . . . . . . . . . 40

8.3 .1 Input Data . . . . . . . . . . . . . . . 40

8.3 .2 GA Parameters . . . . . . . . . . . . . . . . . . 41

8.3.3 Leave-One-Out Cross-Validation . . . . . . . . . . . . . . . . 42

8.3.4 Comparison with ENDEAVOUR and Random Walk (GPEC) . 43

8.3.5 Predicting novel Parkinson's disease genes . . . . . . . . . . 44

8.4 Analysing GA capability to evolve disease gene communities with higher modularity . . . . . . . . . . . . . . . . . 45

9 Conclusions and Future Work $\quad 47$

$\begin{array}{ll}\text { Bibliography } & 57\end{array}$

$\begin{array}{ll}\text { Appendices } & 57\end{array}$

A Sample output of genetic algorithm $\quad 58$

A.1 Generations 1 to $20 \ldots \ldots \ldots \ldots \ldots \ldots \ldots$

A.2 Generations 291 to $300 \ldots \ldots \ldots \ldots \ldots \ldots$

B Samples of generated candidate genes ranking $\quad 84$

B.1 A candidate genes ranking for Breast Cancer . . . . . . . . . . . 84

B.2 A candidate genes ranking for Parkinson's disease . . . . . . 87

$\begin{array}{ll}\text { C Sample disease genes communities } & 91\end{array}$

C.1 Breast Cancer . . . . . . . . . . . . . . . . . . . . . . . . . 91

C.2 Parkinson's disease . . . . . . . . . . . . . . . . . . . 91 


\section{List of Tables}

3.1 Some of the well-known developed disease-gene association methods . 17

8.1 Known disease genes for breast cancer . . . . . . . . . . . . . . 37

8.2 GA Parameter Settings for breast cancer experiment . . . . . . . . 37

8.312 successfully predicted genes out of the 16 known disease genes for breast cancer listed in Table 8.1 . . . . . . . . . . . . . . . . . . . 39

8.4 Analysis and comparison for breast cancer experiment . . . . . . . . . 40

8.5 known disease genes for Parkinson Disease . . . . . . . . . . . . . . . 41

8.6 GA Parameter Settings for Parkinson Disease experiment . . . . . . . 42

8.7 9 Successfully predicted genes out of the 15 known disease genes for Parkinson's Disease listed in Table 8.5 . . . . . . . . . . . . . . . 43

8.8 Analysis and comparison for Parkinson's disease experiment . . . . . 43

8.9 P-values given by t-test . . . . . . . . . . . . . . . . . 45

8.10 Comparing results for tournament size of 1 and $3 \ldots$. . . . . . . 46 


\section{List of Figures}

2.1 Human DNA structure, image from $[17] \ldots \ldots \ldots \ldots$

2.2 General Schema of Gene Expression, image from $[6] \ldots \ldots$

2.3 The most common mutation in genes (SNP), image from [38] . . . 8

2.4 General Schema of Computational Disease Gene Association . . . . . 9

5.1 Partial map of the internet based on the January 15, 2005 data found on opte.org, image from $[85] \ldots \ldots$. . . . . . . . . . . . 22

8.1 averages of best/average fitness values over generations (breast cancer

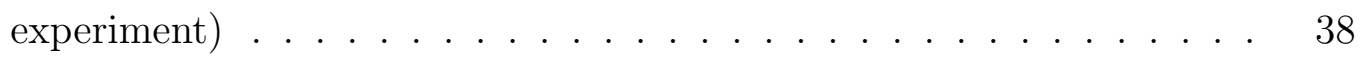

8.2 Averages of best/average fitness values over generations . . . . . . 42

8.3 comparing averages of best/average fitness values over generations while

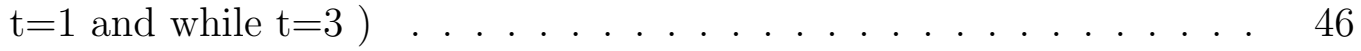




\section{Chapter 1}

\section{Introduction}

Understanding the relationship between genetic diseases and the genes associated with them is an important problem regarding human health. Acquiring more information and knowledge about the particular genes involved with a given disorder can help to devise diagnosis and therapeutic strategies to treat genetic diseases and disorders.

The vast amounts of data created from a large number of high-throughput experiments performed in the last few years has resulted in an unprecedented growth in computational methods to tackle the disease gene association problem. Embedded in available resources including increasingly large genomic databases and vast amounts of relevant literature is evidence to link genes to diseases [77]. The disease-gene association problem is now a major part of bioinformatics, a field which revolves around managing, storing, and analysing the vast amount of genomic data being produced by modern cutting edge science and technology [16].

Computational disease-gene association methods apply all the possible and potential findings throughout years of research in related areas, using whatever useful information can be found in the literature to associate genes with diseases. The most crucial pieces of information for computational disease-gene association methods to link diseases with particular genes is the already established set of known disease genes, i.e. those genes that have already been observed to be involved in a given disease. This is particularly because genes causing the same or similar diseases tend to lie close to one another in the networks in which they interact; this is known as the guilt by association principle which is discussed in Section 2.3.2. The genes which we choose to assess their association with known disease genes are called candidate genes and are acquired by several practical and computational approaches discussed later in this thesis.

Subsequent to years of relevant studies and discoveries, it is now clear that the 
complexity of the interactions among the genes and their products (i.e. proteins, discussed in Chapter 2 ) is considerable. Thus, the disease-gene association problem is no longer a matter of establishing simple connections between diseases and genes, and is an increasingly challenging problem. As a result, a scientific field which is closely related to the disease-gene association problem is the science of complexity, i.e. analysing large complex systems and networks.

In this research, inspired by notions related to community identification in complex networks (discussed in Chapter 5), we devised a genetic algorithm to rank candidate genes in the order of their relevance to the known disease genes. Our genetic algorithm gives our method remarkable capabilities regarding disease-gene association, as discussed in Chapter 6. The preliminary results gained by this approach are satisfactory and comparable with the results given by the most reliable and frequently used frameworks of disease-gene ranking, as seen in Chapter 8.

\section{$1.1 \quad$ Thesis Structure}

The remainder of this thesis is organized as follows. In Chapter 2, we give background knowledge about basic concepts regarding biology and bioinformatics and subsequently we define the computational disease-gene association problem in detail. Chapters 3 and 4 review pertinent studies previously performed. In Chapter 5, we introduce the mothodologis we applied to develop our method, i.e. complex networks and genetic algorithms. Chapter 6 describes the architecture of our method along with the related ideas and motivations to develop it. Chapter 7 outlines the data resources, databases we used to provide the required data for our experiments, and the benchmark tests we used to assess the results procured in our case studies. In Chapter 8 we present two of the experiments performed by our method for breast cancer and Parkinson's Disease and analyze the results. Chapter 9 discusses the potential work one can pursue to continue this research and to make our method more effective. 


\section{Chapter 2}

\section{Background}

\subsection{Bioinformatics: promises and applications}

For the first time, in the late nineteenth century, Gregor Mendel shed light on how characteristics are inherited in living organisms. This led to the appearance of the science of genetics, the study of heredity and genes in living organisms [35]. Genetics was revolutionized in 1953 after the identification of DNA (deoxyribonucleic acidexplained in Section 8.1) as the main framework in which all genetic information is encoded. Scientists observed that changes in the structure of the DNA led to changes in the workings of cells and organisms in health and disease [22]. However, the main revolution occurred in the mid-1990s when the complete nucleotide sequence of the entire human genome was sequenced which includes three billion base-pairs.

What we are encountering now is a vast and ever-increasing flood of genetic data. This has led to the challenging problem of extracting meaningful information and scientific conclusions from the enormous amount of available data. This remarkably requires development of new computational and analytical approaches to uncover new nuggets of information. The scientific field which tries to tackle this ever-challenging problem is bioinformatics. Here, two comprehensive definitions of bioinformatics are provided:

"Bioinformatics is the new science at the interface of molecular biology and computer science seeking to develop better ways to explore, analyze, and understand genetic data [16]".

"Bioinformatics is a new field in which several disciplines work together to manage, store, and analyze the [biological] data being produced around the world. [33]

Throughout a few years of development, bioinformatics can now be divided into several areas, each trying to meet a specific challenge in the area of the bioinformatics. 
The research we report in this thesis is involved in one of these areas, identification of disease-genes, which has recently been tackled via applying various computational and analytical approaches. In this chapter, we introduce this area of research in more detail.

Associating genes with phenotype - "the collection of all of an organism's physical traits [60]" (further details in Section 2.2.3) - has been one of the most demanding research subjects in bioinformatics. One of the major parts of gene-phenotype association research is to associate genes with specific phenotypes, i.e. the genetic diseases. The specific research area dealing with this problem is called the diseasegene association problem, or identification of disease genes, or disease gene prediction/prioritization, i.e. identifying the genes which are in any way involved in the existence of a given genetic disease or disorder, or prioritizing genes in the order of their relevance to diseases.

Associating genes with genetic diseases and disorders is crucial to identify the genetic basis of human diseases. Procuring more information and knowledge about the genes behind a given disorder can lead to more effective diagnosis, improved prognosis, and development of improved therapeutic strategies to treat the genetic diseases and disorders. Bioinformatics tries to tackle the disease-gene association problem via developing computational disease-gene prediction methods. These methods or tools apply the discoveries and findings from years of research in related areas by examining useful information from the literature, to associate genes with diseases [47]. Further details about these methods are provided in Section 2.3 and Chapters 3 and 4 .

\subsection{Basic Biological Concepts}

\subsubsection{Genes, the living organisms' algorithms and software}

Every cell in the human body contains the same DNA sequence, which is composed of a long string of chemicals called nucleotides. There are four types of nucleotides: guanine $(\mathrm{G})$, adenine $(\mathrm{A})$, thymine $(\mathrm{T})$ and cytosine $(\mathrm{C})$. In fact, all the genetic information of the living organisms is encoded in DNA. In humans, DNA contains two long strands (see figure 2.1) each composed of almost 3 billion nucleotides.

Not all parts of DNA are the same. Some parts of the DNA handle more functionalities than the others. In other words, some specific parts of the DNA have been less subjected to mutation and more conserved in the course of evolution (refer to [16], Chapter 8 for more details regarding this). These segments of the DNA which 


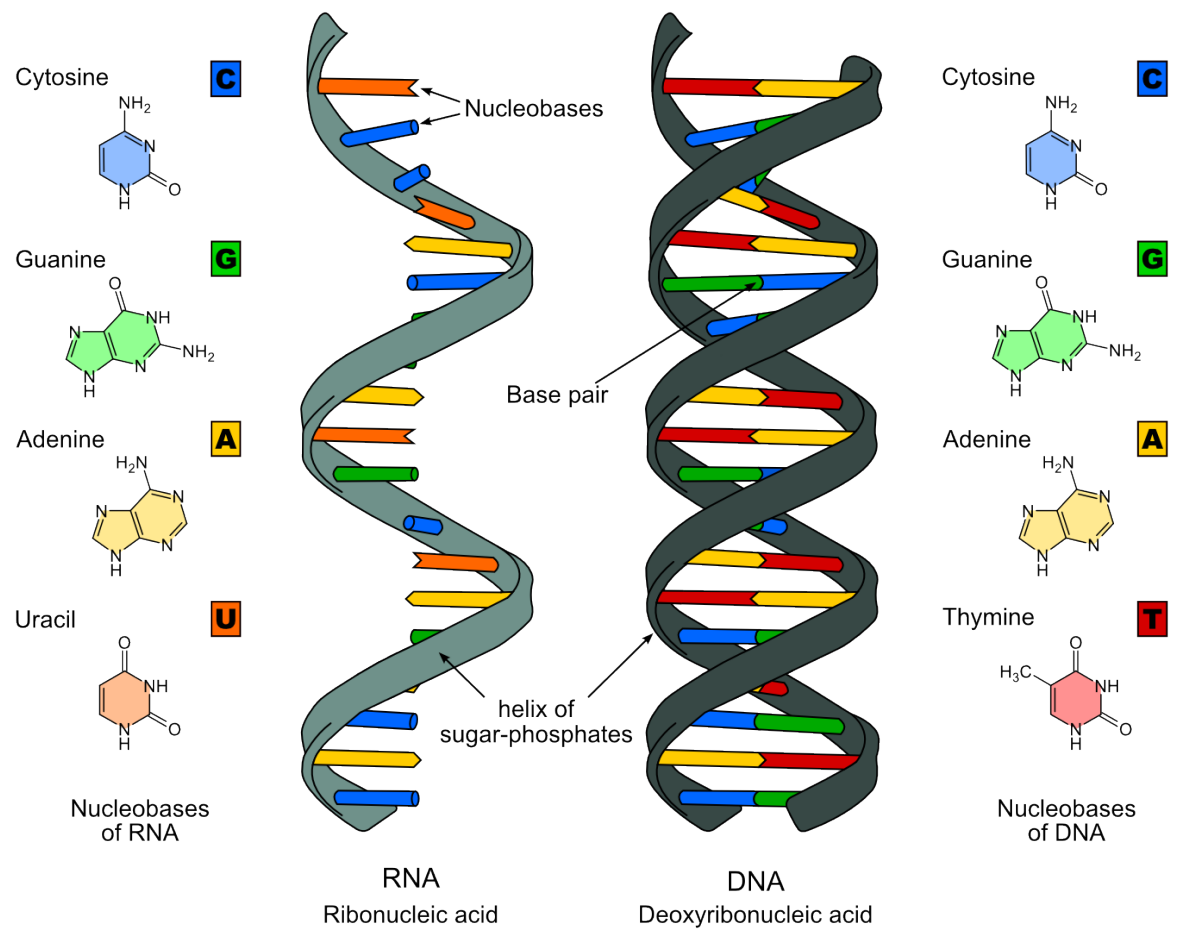

Figure 2.1: Human DNA structure, image from [17]

carry inheritable information about one or more functionalities are called genes [16]. A gene is usually defined as the molecular unit of heredity of a living organism [33]. Every cell of every individual contains two complete sets of genes, with each set lying on one of the two strands of the DNA. If one of the sets does not function correctly, the other one may take over the functionalities. For further details about how genes work, refer to [16] and [33].

\subsubsection{The living organisms hardware: the proteins}

How is the genetic coded information inside these important functional segments of the DNA, i.e. the genes, put into action in humans or other living species? More precisely, how do different cells in the same organism conduct their own specific functions using the same genetic code they all have? This question can also be rephrased as in [16] "How do cells respond to changing environmental conditions using the genetic instructions"?

The encoded genetic instructions in the genes are used to produce proteins, the large biological molecules performing different functionalities within living organisms. In fact, genes can be interpreted as algorithms which develop proteins, the most important functional units of every living organism. The process by which genes 
are applied to develop proteins is called gene expression (figure 2.2). When the instructions in a give gene are applied to make proteins, it is said that the gene is expressed. Although all cells carry all of the genome (i.e. all the genes, or the entire DNA), only a small fraction of the genome is expressed in every cell with regard to the specific tasks and functionalities each cell performs (nerve cell, muscle cell, bone cell, etc). As a matter of fact:

"In each cell type, a specific set of genes is activated, and the proteins they encode give the cell the characteristics of a muscle cell, bone cell, nerve cell and so on. Furthermore, cells alter their patterns of gene expression depending on their environment in order to respond to nutrients, temperature, hormones, infectious agents, and so on. [16].

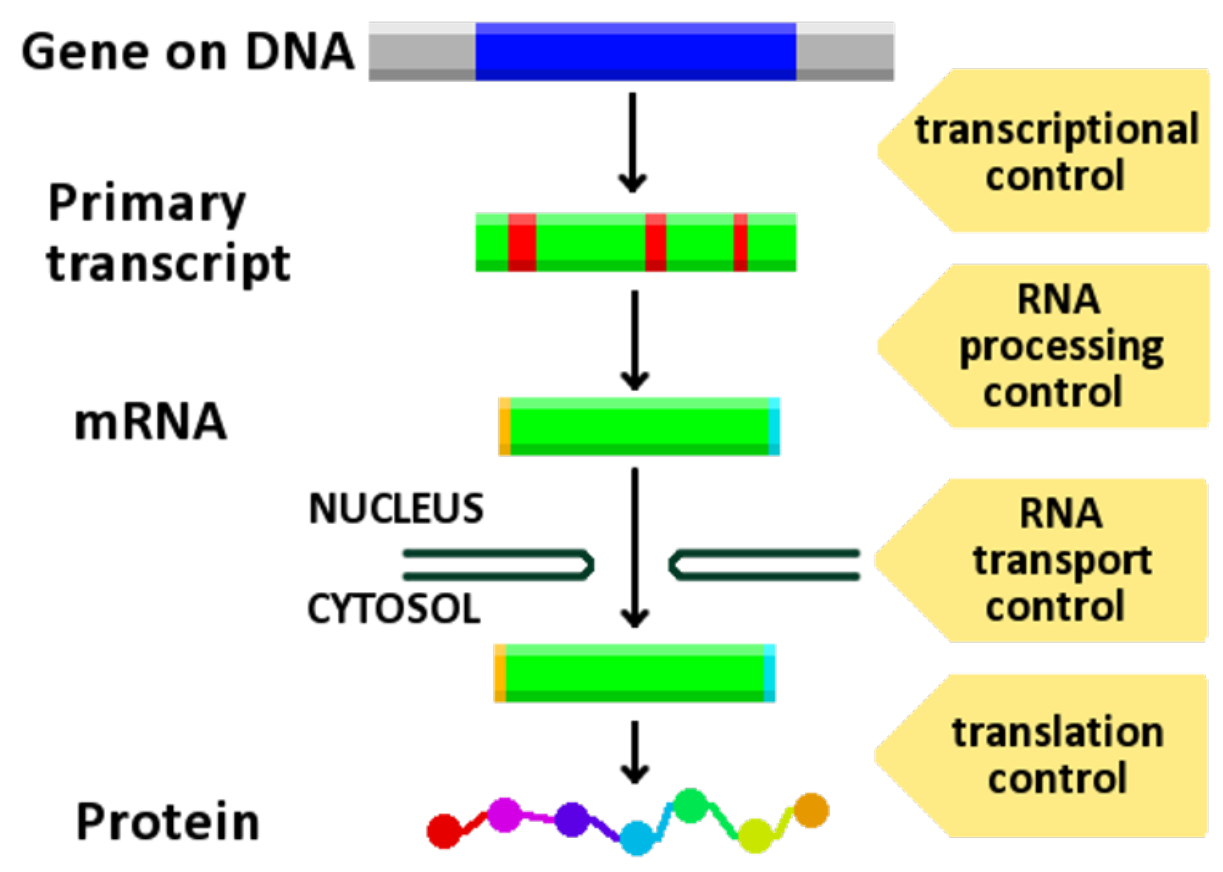

Figure 2.2: General Schema of Gene Expression , image from [6]

\subsubsection{Genetic disease as a type of phenotype}

As a conclusion of what has been discussed in previous sections, it is fairly true to suggest that all the functions of living organisms like humans depend on the proteins. Hundreds of thousands of proteins in our body carry out thousands of chemical reactions required for life [9]. 
The question which might come to the mind now is this: how do proteins lead to who we are? "Who we are" is a complicated question which can never be answered clearly. But from the biological perspective, one may answer this question as: "we are a huge set of multitudes of various phenotypes". Phenotypes are sets of observable properties and characteristics of the living organisms at different levels. For instance, a phenotype at the cellular level can be the type of cell, the shape of the cell, or other observable properties of the cell. A phenotype at a higher level can be the human skin color, eyebrow shape, eye color and so on. A phenotype can also be as complex as being alive. The fact that we are alive at a given moment is a complicated phenotype depending on very many processes, interactions and other simpler phenotypes. Thus, at any given moment, we are a set of a myriad of different phenotypes at different levels. All these phenotypes come about subsequent to interactions of an extraordinarily huge number of proteins interacting with each other in a real time network all over the cells of our bodies. This gigantic network across all the cells is referred as the human interactome. The human interactome is scientifically believed to be one of the most complex networks containing approximately 25000 protein-encoding genes, and also a high number of unknown and undefined proteins and their interactions [9]. The number of nodes of the network including genes and their products such as proteins is estimated to exceed one hundred thousand [9]. The number of functionally relevant interactions between the components of this network, representing the links of the interactome, is expected to be much larger and remains largely unknown [9].

Unfortunately, phenotypes are not always handy or pretty. They can sometimes be undesirable and even destructive. A phenotype can also be a genetic disease or disorder. The most common reason for genetic disorders is changes or mutations happening in the genes. The altered gene is called a mutated gene. Mutation in the DNA is not a rare incident. As most parts of human DNA are not functionally important, mutations in these parts do not usually lead to harmful consequences. However, if mutation occurs in a functional part of the DNA - inside the genes which handle some functionalities - it may adversely affect some crucial processes required for life, consequently causing genetic diseases. The most common mutation causing genetic disease is $S N P$ (single nucleotide polymorphism) in which one single nucleotide $\mathrm{A}, \mathrm{T}, \mathrm{C}$ or $\mathrm{G}$ is mutated and replaced by another one (see figure 2.3). If this occurs in a coding area of DNA (genes) it may affect the protein function and consequently lead to genetic disorders. 


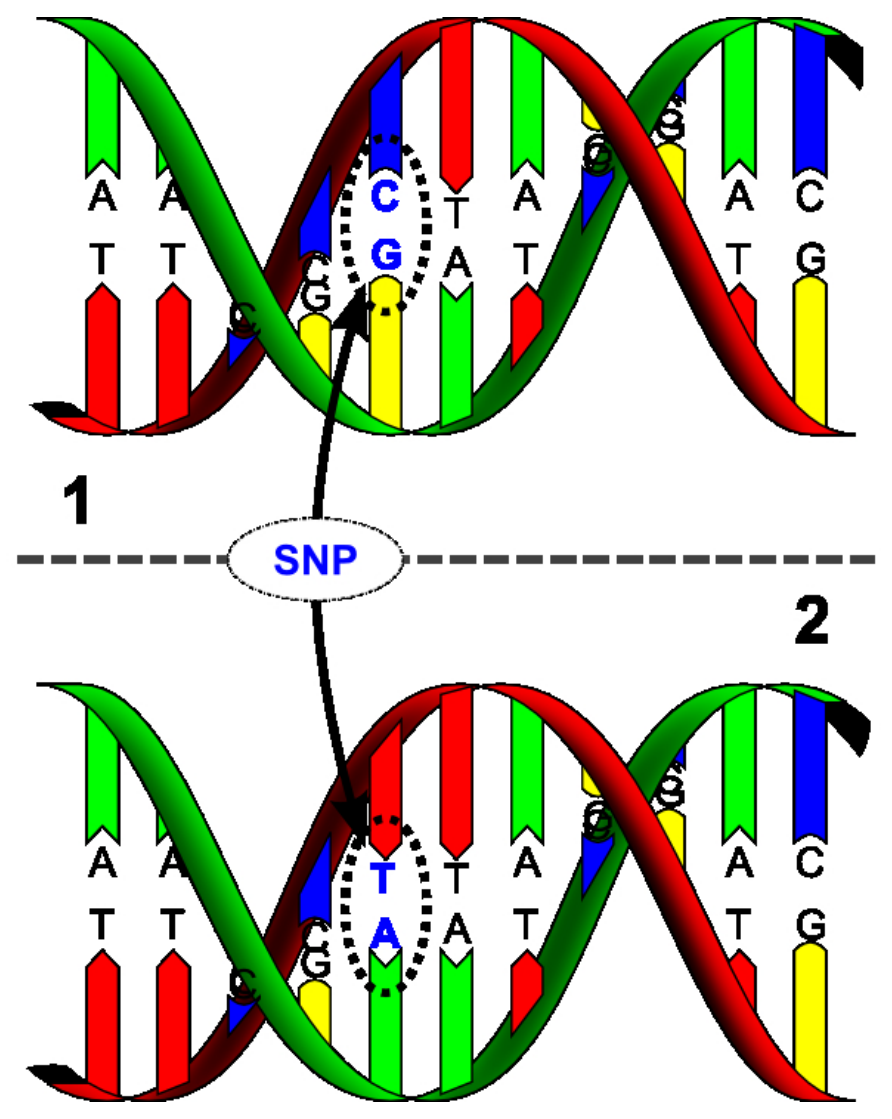

Figure 2.3: The most common mutation in genes (SNP), image from [38]

\subsection{Disease-Gene Association Problem}

As discussed earlier, "The expression of a subset of genes and their complex and orchestrated interactions and networks confer specific properties on the cell, thereby establishing its phenotype[16]. Associating genes with phenotypes has been one of the most demanding research subjects in bioinformatics. This problem has always been one of the trickiest problems consequent to the complexity of the interaction network between the genome and the phenotypes. One specific gene-phenotype association problem is to associate genes with specific phenotypes, i.e. genetic disease. The research area tackling this problem is called the disease-gene association problem or identification of disease genes, or disease gene prediction/prioritization, i.e. identifying the genes which are in any way involved in the existence of the genetic disease or disorder. In this thesis, all these titles may be interchangeably used. 


\subsubsection{Bioinformatics and Computational Disease-Gene Asso- ciation}

How does bioinformatics tackle the disease-gene prediction problem? More precisely, how can one develop computational and analytical methods to meet the disease-gene association challenge? Understanding the complex correlation between genes and proteins requires processing of vast amount of data from a wide variety of genomic data sources. "Thus, computational tools have become critical for the integration, representation and visualization of heterogeneous biomedical data" [50].

Figure 2.4 depicts the general schema of computational disease gene prediction. To be extremely general, computational disease-gene association methods apply all the possible and potential findings throughout years of research in related areas, using whatever useful information can be found in the literature to associate genes with diseases.

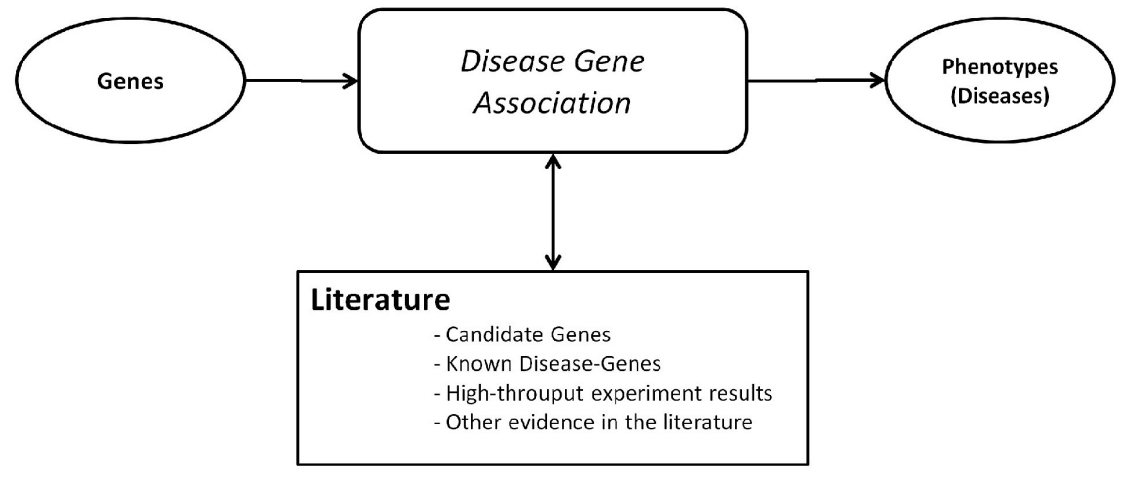

Figure 2.4: General Schema of Computational Disease Gene Association

Disease-gene prediction methods choose potential disease genes from a set of candidate genes usually determined by different experimental and other computational methods including Genomewide Association Studies (GWAS) and Linkage Analysis. In Genomewide Association Studies hundreds of thousands of SNPs (Single Nucleotide Polymorphisms) are statistically investigated for association with genetic disease in hundreds of thousands of individuals. Linkage Analysis typically associates certain chromosomal loci (linkage intervals) with a particular disease phenotype [37] and it consists of expensive practical experiments performed in the laboratory to determine loci on chromosomes which have the tendency to be inherited alongside the known disease genes or mutated genes which are believed to be involved in diseases. For 
further information on Genomewide Association Studies and Linkage Analysis, see [23].

\subsubsection{Known Disease Genes and the Principle of Guilt by Association}

One of the most important pieces of information in the literature which can aid computational disease gene prediction methods to associate diseases with genes is the already established set of known disease genes, i.e. those genes that have already been observed to be involved in a given disease. Our knowledge about the known disease genes can be crucial for disease gene prediction methods to identify new novel disease genes. This is mainly because genes causing the same or similar diseases tend to lie close to one another in the networks in which they interact. For example, genes related to the same disease are known to have protein products that physically interact. Also, previous studies have shown that mutations in multiple proteins that form a protein complex may lead to the same disease phenotype. This is called the principle of guilt by association. According to this principle, disease genes for the same disease tend to interact with one another, or in collaboration with each other, in the cell. This is also called the modular nature of genetic disease, i.e. the concept that various genes involved in the same phenotypes work together in a single biological module [64].

\subsubsection{Disease-Gene Association as a Complex Problem}

The complexity of the interactions among the genes is significant. A remarkable amount of interactions have been discovered, subsequent to years of related discoveries and studies, including network-based data resources, i.e. resources recording the interactions among the genes in different aspects such as protein-protein interaction networks, gene co-expression networks, metabolic pathways and functional annotations. Such network-based resources have become preferred data resources to associate diseases with genes [9]. Disease-gene association is no longer a matter of establishing simple connections between known disease genes and candidate genes using a set of straight-forward evidence. It is crucial to note that complex phenotypes are far beyond the sum of independent effects of the individual genes. As a matter of fact, "the impact of a specific genetic abnormality is not restricted to the activity of the gene product that carries it, but can spread along the links of the network, and alter the activity of gene products that carry no defects" [9]. Furthermore, "the phenotypic 
impact of a defect is not determined only by the known function of the mutated gene, but also by the functions of components with which the gene and its products interact and of their interaction partners, i.e., by its network context [9]". From these key assumptions, it must be concluded that genetic disorders must not be simply considered as a consequence of mutation(s) in a single gene. Instead, the disease phenotype is a reflection of various components (genes, proteins, etc.) interacting in a complex network. We precisely define complex networks with examples in Chapter 5.

Classic forms of disease gene prediction approaches, in the absence of considering the notion of complex networks, were not able to process the myriad number of interactions among the genes and proteins and identify the genes based on the complex behavioral patterns discovered in the networks. It has been shown that it is necessary to process and observe the interactions among the complex systems components in order to find facts and draw conclusions about these systems. As stated in [60], "Many of the ideas are disguised behind the interactions among the components, and not inside the component". As Aristotle said in 335-323 BCE, "the whole is more than the sum of its parts" [34]. 


\section{Chapter 3}

\section{Literature Review of Disease-Gene Association}

\subsection{Definition of Computational Disease-Gene As- sociation}

Based on what we have learned in previous chapters, a brief, general and formal description of disease-gene association should be provided. There are many appropriate definitions suggested by different resources, however, the one provided in [77] can barely be surpassed:

"Genome-wide association analyses on large patient cohorts are generating large sets of candidate disease genes. This is coupled with the availability of ever increasing genomic databases and a rapidly expanding repository of biomedical literature. [Using any computational and analytical techniques], Computational approaches to diseasegene association attempt to harness these data sources to identify the most likely disease gene candidates for further empirical analysis by translational researchers, resulting in efficient identification of genes of diagnostic, prognostic, and therapeutic value" [77].

What is the accurate meaning of the word association in disease-gene association? Basically, according to the pertinent literature, there are two main approaches for association of diseases with the genes [68]. The results obtained by a disease gene association method could be either a prioritization of the candidate genes, i.e. a ranking of the candidate genes based on the likelihood of their involvement, or a selection of the candidate genes, i.e. a reduced set of candidate genes which are of higher priority to be studied than other candidates, or that are more noteworthy 
in some way. In many cases, there is no strict difference between these two prediction approaches. Usually, a prioritization-based method is developed over an already developed selection-based method of prediction. Prioritization based disease-gene association methods are also called disease-gene prioritization methods.

\subsection{Classification of Existing Evidence for Disease- Gene Prediction in the Literature}

In this section, we discuss several types of existing evidence in the literature frequently being applied to associate diseases with genes. This evidence can be roughly classified into text-mining of the literature, functional evidence and pathways, phenotypic evidence, inherent gene properties, sequencing data and mutation evidence, protein-protein interaction, gene expression data, and some other types of evidence.

\subsubsection{Text Mining of the Literature}

Natural language processing and text-mining of biomedical and bioinformatics literature is amongst the first effective approaches being used to tackle the disease-gene association problem. There are enormous amounts of knowledge recorded by data resources such as PubMed [1] and OMIM [39] containing a multitude of biomedical abstracts and studies. These data resources are freely and easily available and the information and knowledge recorded by them can be used as input for text-mining, natural language processing, or other computational methods to discover significant links between genetic diseases and the genes. By 2013, the PubMed database recorded 22 million citations from more than 24000 journals [68]. OMIM has recorded thousands of phenotype and disease genes descriptions.

In an extensive study performed at Stanford University, Altman et al [5] collected opinions from the leading scientists about how text mining can provide better access to the biological literature. They also provided worthwhile instructions on how to apply text mining techniques over different types of literature data resources to gather evidence for different purposes. In one of the first attempts in using text mining of the bioinformatics literature, Blaschke et al [10] designed a system for automatic detection of protein-protein interaction extracted from scientific abstracts. JB Laurila et al [54] studied mutations' impact on protein properties using text mining of the literature to find novel mutations leading to diseases. Özgür et al [66] introduced an automatic approach based on text mining and network analysis to predict gene-disease 
associations.

\subsubsection{Functional Evidence and Pathways}

Different functionalities performed by different types of cells are provided subsequent to a series of chemical events called biological pathways. A biological pathway is defined as a series of actions and interactions among the molecules in the cell which lead to a specific phenotype. The suspect gene is likely to be a member of the same molecular pathways as other disease genes. In other words, there is a good chance that the unknown genes involved in the same pathway as other known disease genes are connected to the disease in one way or another [11]. Two of the most reliable and frequently used databases which constantly record functional annotations and pathways of the genes are Gene Ontology (GO) [7] and Kyoto Encyclopedia of Genes and Genomes (KEGG) [49].

Frances S Turner et al [83] developed POCUS, a computational approach to prioritize candidate disease genes based on their functional similarities.

Apart from all capabilities of the functional annotations for disease gene prediction, there is a key downside with the use of functional annotation for disease gene prediction as there are still many unverified and unknown functional pathways. This makes disease gene identification biased towards the better-studied functionalities and pathways [68].

\subsubsection{Phenotypic Evidence}

For some unknown genetic diseases, there exists a major lack of knowledge and information about the molecular basis of the candidate genes. In such cases, one of the possible approaches to determine associations between candidate genes and knowndisease genes is to use the phenotypic relationships of the genes. The use of phenotypic evidence for disease-gene association is based on the observation that genes involved with similar phenotypes often participate in the same interaction networks [11]. In other words, genes involved in a well-studied disease could also be involved in an unknown disease with similar symptoms. However, this is not always true [68].

One remarkable database which describes relationships between phenotypes is MimMiner [79]. J Freudenberg et al [29] developed a statistical method that used MimMiner for prediction of disease relevant human genes from the phenotypic appearance of a query disease. They tested their framework on colorectal neoplasms disease family. 


\subsubsection{Inherent Gene Properties}

Inherent (intrinsic) properties of genes and proteins such as gene or protein length, degree of conservation, phylogenetic breadth and so on, can also provide some clues to discover possible correlation between genetic disorders and genes, as some of these properties may statistically differ between disease genes and genes not involved in disease [68].

López et al [56] used inherent gene and protein properties including length of amino acid sequence, phylogenetic extent, and conservation degree to develop a computational method to detect disease genes. Adie et al [3] examined a variety of intrinsic sequence-based gene features and found that for many of the cases there are significant discrepancies between the sets of genes known to be involved in genetic diseases and those not known to be involved in diseases. Based on these genes' features, they developed PROSPECTR, an automatic classifier, which ranks genes in the order of likelihood of involvement in disease.

\subsubsection{Sequencing Data and Mutation Evidence}

Analytical processes and investigations of the genes' mutations can be helpful to find meaningful patterns regarding the mutations existing in disease genes. Such investigations can give clues to find genes mutated in a similar way which can be added to the candidate genes list. If there exist a large number of candidate genes for a given disease, a high number of variations in sequences can be observed. For instance, Tarpey et al. [76] studied 718 candidate genes on the X chromosome from 208 families for mental retardation. They discovered mutations in three novel genes based on mutations in several known disease genes. Najmabadi $\mathrm{H}$ et al. [61] studied 136 families with autosomal-recessive intellectual disability. They successfully discovered 50 novel candidate genes based on the identified mutations in 23 known disease genes.

Although using sequencing data has been successfully used to predict novel disease genes, however, it still cannot identify many of the genetic causes of the disease due to a shortage of knowledge about the accurate sequence of the candidate genes [68].

\subsubsection{Protein-Protein Interaction}

Protein-protein interaction networks (PPI) are networks representing physical interactions between proteins; this is the most frequently used and powerful type of evidence for disease gene prediction [62], [14]. In relation to the guilt by association 
principle (see Section 2.3.2), proteins which physically interact are more likely to be involved in the same functionality or phenotype. Therefore, harmful alteration of any of the proteins interacting in the same functional module (which is probably caused by deleterious mutations in the gene expressing the protein), can adversely change the functionality or phenotype, and consequently lead to the same disorder [62].

Among the most trusted and frequently used databases recording PPI are STRING [46] and the Human Protein Reference Database (HPRD) [70]. These data resources have recorded myriad observed interactions from various organisms.

In some of the earliest studies to use PPI for disease-gene association, J Xu et al [87] created a classifier based on several topological features of the underlying proteinprotein interaction network to predict disease genes and M Oti et al [65] used more than 70000 protein-protein interactions between close to 11000 human proteins to offer sets of candidate genes for more than 380 genetic diseases.

There have been many efforts to use PPI for disease-gene associations. In this research, we used PPI to associate disease with genes. Chapter 4 reviews some remarkable studies of using PPI for disease-gene association.

\subsubsection{Gene Expression Data}

As mentioned in Section 2.2.2, gene expression is the process of transcribing and translating a gene to yield a protein product [16].

There are two key factors affecting how a gene is expressed:

- Cell type: Although all the cells have the whole genome, only a fraction of the genes is activated (expressed) in every cell depending on the type of cell, which is why different cells in the human body perform different tasks.

- Environment: Cells change their pattern of gene expression depending on their environment in order to properly respond to nutrients, temperature, hormones, infectious agents and attackers, and so on [16].

It is intuitively obvious that disease genes must be expressed differently than normal genes, as if they expressed in the same way then they would never lead to diseases. The microarray technology first developed in 1995 by Schena et al. [75] now makes it possible to relatively measure the gene expression of a given gene. Using this technology, researchers can compare gene expression of genes in different cells and in different environmental circumstances. Accordingly, it is possible to compare the expression of the disease genes to normal genes and make links between disease genes 
and candidate disease genes by measuring the similarity between their expressions on microarrays.

\subsection{Applying a Fusion of Different Evidence and Resources}

Many of the recently developed disease gene prediction approaches have tried to take advantages of multiple evidence at the same time in order to provide more accurate and reliable results.

There are two basic challenges involved with the use of several data resources regarding different evidence categories. First, several data resources with different properties have to be incorporated into the single coherent computational framework. Second, for prioritization-based approaches (see 3.1), there has to be a single integrated prioritization formula based on the different metrics connected to different data resources.

Table 3.1 lists a number of previous works using different evidence at the same time.

Table 3.1: Some of the well-known developed disease-gene association methods

\begin{tabular}{|c||c|c|}
\hline Method & evidence type & reference \\
\hline \hline CIPHER & phenotype, PPI & {$[86]$} \\
\hline ENDEAVOUR & expression, PPI, functional, inherent, text mining & {$[4]$} \\
\hline GFINDER & phenotype, expression & {$[59]$} \\
\hline CAESAR & expression, functional, inherent, text mining & {$[31]$} \\
\hline CGPRIO & sequence, functional, PPI, inherent & {$[86]$} \\
\hline GENESEEKER & expression, functional, text mining, phenotype & {$[78]$} \\
\hline
\end{tabular}




\section{Chapter 4}

\section{Review of using PPI for Disease-Gene Association}

As discussed in Section 2.3.3, genetic disorders are normally complex and are caused by several genes involved in various biological processes [37]. The application of protein-protein interaction networks has remarkably extended our knowledge about the genetic causes of genetic diseases [37], [50], [45].

As we applied protein-protein interaction information for our disease-gene association method, we now provide a literature review of using this type of evidence.

Disease-gene association methods using PPI can be roughly classified into the following categories: neighbourhood-based algorithms, random walk algorithms, and graph partitioning algorithms.

\subsection{Neighbourhood-based Algorithms}

In neighbourhood-based approaches, the main assumption is that the proteins in the neighborhood of the proteins expressed by disease-causing genes are likely to be involved in either the same or a similar disease [45]. In other words, these methods presume that two genes closer to one another in the underlying protein protein interaction network often lead to more similar phenotypes [86].

A good example of such methods is CIPHER [86], which gives scores to candidate genes based on the closeness of the proteins derived from them to the proteins derived from known disease genes, in the protein-protein interaction network. Dezső et al [20] ranked the candidate genes with respect to the shortest distance between disease genes in their interaction network. 


\subsection{Random Walk Algorithms}

These methods basically use a random walk on the interaction network starting from one of the known disease genes with some probability back to the one of initial nodes. In other words, at each step of the walk, there is a probability of $r$ that the walk restarts and then starts again from one of the nodes associated with known disease genes. A probability vector is then created containing the probability of randomly visiting a node in a given time. The prioritization of the candidate genes is made according to the visitation probability being more than a specific threshold.

To see sample studies on disease-gene association using random walk on proteinprotein interaction networks see [52], [80], [14] and [55].

\subsection{Graph Partitioning Algorithms}

As we already know, most cellular functionalities are conducted not by individual proteins, but by a group of functionally associated proteins, referred to as modules [2]. In protein-protein interaction networks, modules are considered as densely interconnected nodes, known as communities or clusters.

Using a community identification method named Clique Percolation Clustering [19] PF Jonsson et al [48] showed that proteins expressed from known cancer genes have different network properties than proteins not observed as being involved in cancers. For instance, they observed that cancer proteins show an increase in the number of proteins they interact with.

S.Navlakha et al [63] proposed a graph summarization technique (named GS) based on graph compression to cluster protein interaction graphs into biologically related modules. 


\section{Chapter 5}

\section{Methodologies}

Before seeing the details of our computational approach for disease-gene association, one should be generally familiar with complex networks, community identification problem in complex networks, and genetic algorithms. In this chapter we provide an overview of these methodologies.

\subsection{Science of Complexity}

How do hundreds of thousands of proteins interact and form various complex functionalities and phenotypes of the human body? How do a huge number of cells organize themselves to be our nervous system or our skin? Or, "how do independent members of an economy, each working chiefly for its own gain, produce complex but structural global markets; or, most mysteriously, how do the phenomena we call intelligence and consciousness emerge from non-intelligent, non-conscious material substrates?" [60].

Many of the systems such as insect colonies, the brain, the immune system of our body, the economy, the world wide web, the biological networks such as genetic interactions and protein interactions, etc. are considered to be complex systems. Complex Systems or the Science of Complexity is "an interdisciplinary field of research that seeks to explain how large numbers of relatively simple entities organize themselves, without the benefit of any central controller, into a collective whole that creates patterns, uses information, and, in some cases, evolves and learns" [60].

As discussed in Section 2.3.3, genetic diseases and disorders occur consequent to defects in the complex network in which genes (i.e. their products, the proteins) interact with one another. Therefore, one should learn concepts concerning analysis of complex systems prior to studying genetic interaction networks in order to study genetic disorders. 


\subsubsection{Complex Networks}

All complex systems are composed of large networks of individual components (i.e genes, proteins, websites, neurons, human individuals, etc.), each interacting with other components based on relatively simple rules. The whole collection of interactions of vast number of components leads to complex patterns and behaviours (see Section 2.3.3). Hence, complex systems are usually modelled via complex networks, which are large, dynamic graphs [81] in which nodes represent the complex system's components, and the edges replicate interactions among them. Generally, the term complex network applies to any large graph corresponding to a complex system [81].

Protein-protein interaction networks are complex networks in which myriads of proteins interact to one another to form different functionalities and phenotypes of living organisms. In human protein-protein interaction network (human interactome) more than one hundred thousands nodes interact through largely unknown number of links (which is believed to be much larger than the number of noes). For instance, refer to figure 1 in [51] to see a visualization of a part of the human protein protein interaction network.

\subsubsection{Community Identification Problem in Complex Net- works}

Complex networks have a tendency to form different communities, usually each pursuing different functionality working in sync with one another [73]. Some examples include different regions of the brain, web pages about the same or similar topics on the World Wide Web, or proteins interacting with one another to perform a specific function. The network inside the communities is relatively dense compared to the parts of the network residing outside the communities. For example, see Figure 5.1 which depicts partial map of the Internet based on available data on January 15, 2005. In this network nodes represent IP addresses and edges represent interactions between them. As it can be seen, some parts of the network are denser than the others. This parts can be matched to communities of IPs which closely work with one another.

There has not been an agreement on the precise definition of a community in complex networks [69]. Descriptively, communities are sub-graphs with a higher level of internal interconnection than other sub-graphs of the network [53]. Based on how great a difference there is between the number of internal and external connections of a given community, the community can be considered a weak community or a strong 
one.

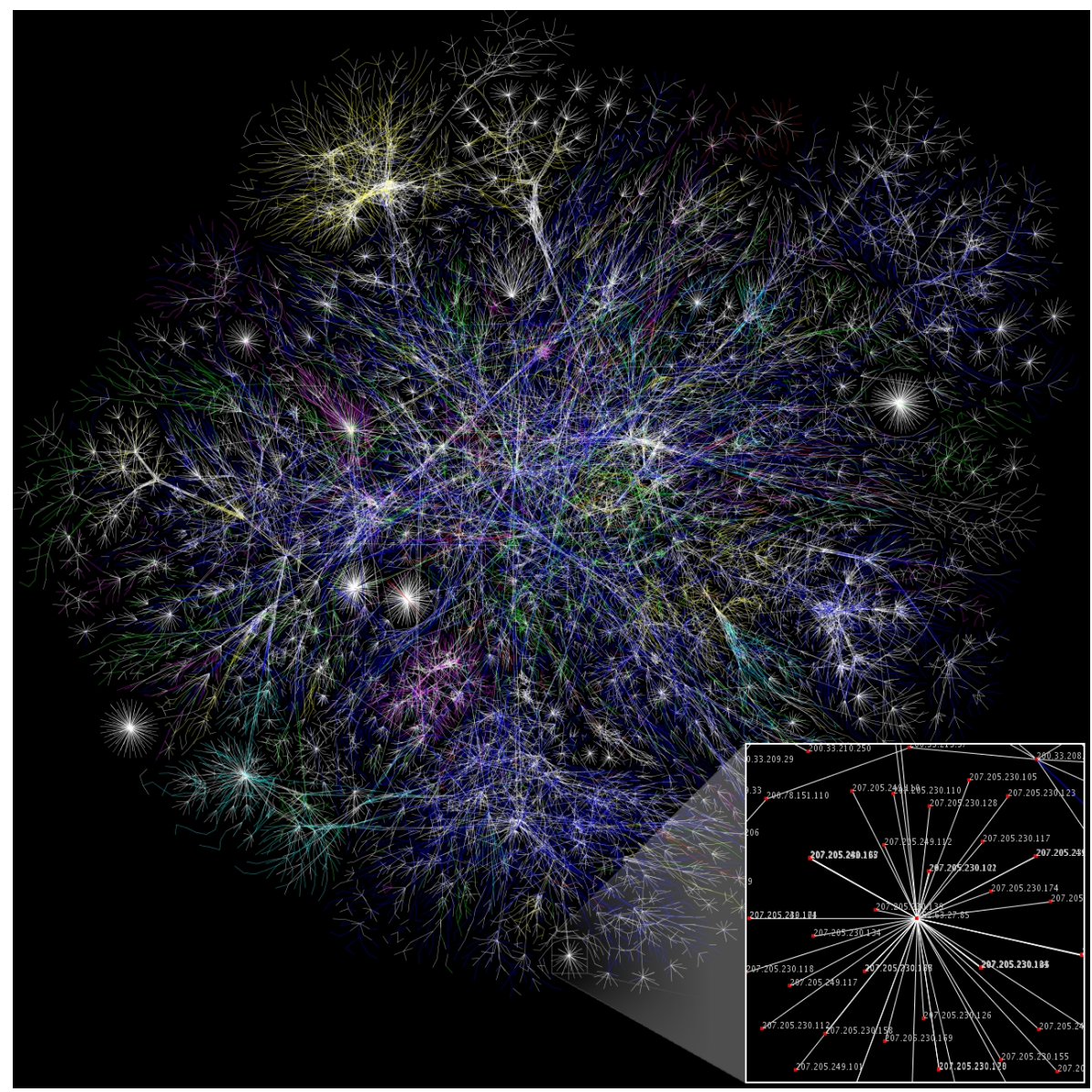

Figure 5.1: Partial map of the internet based on the January 15, 2005 data found on opte.org, image from [85]

In 2002, the field of complex network community identification was first studied by Newman and Girvan [32]. The community identification problem contains within it two subproblems: (1) given a network, identify all communities working in sync with one another in the network, and (2) given a node or a group of nodes, identify the community or communities to which these nodes belong. Both subproblems have been shown to be NP-complete [81]. Detailed discussions about why the community detection problem in graphs is NP-complete are provided in [27]. The latter subproblem is little studied in comparison to the former one [81].

The community identification problem has been studied by several researchers from different fields of science including physics, statistics and computer science. To see a broad and detailed review of community identification methods in complex networks refer to [13], [27] and [28]. 


\section{Community Identification in Biological Networks}

There have been a number of algorithms and frameworks developed so far for detecting communities and modules in biological networks, especially protein-proteinprotein interaction networks. For instances, G.D.Bader et al [8] developed MCODE, an automated framework for finding modular complexes in protein interaction networks using a depth first search algorithm starting at nodes belonging to proteins under study. A.J.Enright et al [22] presented an approach called TRIBE-MCL for clustering of proteins interacting in PPI into different families using a Markov cluster (MCL) [21] algorithm. See Section 4.3 for two other examples of community identification methods on PPI.

As a part of our research, we developed a novel computational approach to identify communities in different types of genetic interaction networks such as PPI, with the use of a genetic algorithm. We developed this approach to associate diseases with particular genes. See Chapter 6 for further details.

\subsubsection{Disease-Gene Association as a Community Identifica- tion Problem}

Different phenotypes including diseases are fairly likely to be assigned to a group of nodes (i.e. genes, RNA molecules, proteins and other distinct cellular components) working closely with one another as a community of the gigantic complex network of the human interactome, a network which is believed to contain some 25000 proteinencoding genes and a still higher number of unknown and undefined proteins and their interactions [9].

From the modern viewpoint of genetics (referred to as the molecular revolution [60]), phenotype-gene association including disease-gene association can be redefined as: given a number of genes known to be involved in a specific phenotype (genetic disease), computationally examine the currently available human interactome (PPI, co-expression, pathway interaction etc.) in the vicinity of the known disease genes to detect the community or communities in which these genes closely interact with one another. Discovering such communities sheds light on genes closely working with known disease genes and steers us towards predicting new disease genes (see Section 2.3.2). Therefore, with this viewpoint of the problem, computational disease gene prediction perfectly matches the second sub-problem of community identification mentioned in Section 5.1.2. 


\subsection{What is a Genetic Algorithm?}

Genetic Algorithms (GA), which are among the most common evolutionary computational approaches, have been proven to be effective in finding near-optimal solutions for many NP-hard problems [60]. The main idea of evolutionary computational methods, including GAs, is to mimic evolution and the Darwinian natural selection process to find a solution (close enough to optimal) for a given computational problem. The first serious effort to apply GAs to practical problems is work done by John Holland and his students at the University of Michigan in the 1970s [60].

Generally, a genetic algorithm contains the following key elements:

- A population of candidate solutions for the problem at hand. These candidate solutions are also called chromosomes or individuals.

- A fitness function that takes a candidate solution and evaluates its fitness value. The fitness value measures how good the solution is for the given problem.

- A crossover operator that is a genetic operator applied on two or more individuals (chromosomes) for offspring reproduction.

- A mutation operator that mimics possible mutations while new individuals are reproduced.

A general recipe for a Genetic Algorithm can be described as having the following steps:

1. Generate an initial population of candidate solutions. This initial population can be generated in many ways but often it is simply a set of random valid candidate solutions.

2. Fitness evaluation: measure the fitness of each individual in the current generation's population.

3. If there is a solution in the population with the desired fitness value, or if the number of generations created so far has reached the predefined maximum number of generations, then the program stops.

4. Tournament selection: randomly select a number (tournament size) of the individuals and select a number (usually two) of them to be the parents reproducing individuals for the next generation. 
5. Generate the population for the next generation: all selected parents in step 4 utilize the crossover operator and possibly the mutation operator to create offspring which will become the individuals of the next generation (new population). This repeats until the new population becomes full.

6. Go to 2.

Applying a genetic algorithm (GA), we propose a new computational approach to tackle the disease gene association problem. We will clarify the technical details of our GA-based disease gene prediction method in Chapter 6. 


\section{Chapter 6}

\section{The Design of Our Computational Approach}

\subsection{General Idea}

Prior to clarifying the technical details of our GA-based disease gene prediction method, we first review its general properties.

Using a genetic algorithm, our computational method tries to evolve communities containing the set of potential disease genes likely to be involved in a given genetic disease. Having a set of known disease genes already believed to be involved in a disease, we first obtain a protein-protein interaction network containing all the selected known disease genes. All the other genes inside the procured PPI network are then considered as candidate disease genes as they lie in the vicinity of the known disease genes in the human interactome. Our method attempts to find communities of potential disease genes strongly working with one another and with the set of known disease genes.

In our GA-based approach, each individual within a population is a candidate community of genes that may be involved in a given disease. The initial population consists of individuals that are simply randomly-created communities. At each generation, the GA tries to evolve individuals (communities) that have a relatively high degree of collaboration between the known disease genes and the other genes within the individual (community), and that also that have a high degree of collaboration among all the genes inside the individual (community). The more collaborative the genes are in the evolved individual (community) with the known disease genes and with one another, the more likely it is that the individual (community) contains genes 
potentially involved in the disease under study. Intuitively, in our method, the GA tries to find subnetworks of the interaction network that are as modular and collaborative as possible, where the subnetworks also contain the known disease genes.

\subsection{Motivations and Ideas}

\subsubsection{Why use a Community Identification Approach?}

There are still many unknown interactions among human genes/proteins in the human interactome, however, despite the considerable noise and incompleteness of protein interaction data, proteins relevant to the same/similar phenotype/biological processes tend to lie near one another within the network (see Section 2.3.2). Thus, community identification approaches can still be effective for predicting novel disease gens existing near the already known disease genes in the human interactome. As discussed in Section 4.3, there have been few successful studies to find communities and clusters in protein-protein interaction networks to associate novel genes with phenotypes, diseases and different biological processes, but this is still a less studied approach compared to other approaches to tackle the disease-gene association problem specifically [81]. In other problems of Bioinformatics, finding and studying protein-protein interaction network modules has led to noteworthy discoveries in physical, logical and evolutionary organization of the cell [63]. (See, for example, [12], [36], [41], [42] and [72]).

\subsubsection{Why use a GA for Community Identification?}

As discussed earlier, there have been quite a few computational approaches developed for community identification in complex networks in general, and more specifically to detect communities in PPI networks (see Sections 4.3 and 5.1.2). One might ask why a GA has been chosen to develop a community identification approach for disease gene association. However, it should be noticed, the computational approach we have devised in this research is not purely a community identification method. As a matter of fact, it focuses on both community identification and disease-gene ranking principles at the same time. In this part, we elaborate the advantages and unique features the GA has given to our computational disease-gene association approach.

Firstly, we tries to evolve communities of disease genes, and not only a single community. Our method works with the populations of various communities (GA populations) which makes it able to process plenty of different potential communities 
to assess their collaboration with the known disease genes set. This creates a competitive environment for many evolving genes communities and the ranking for a given gene depends on all of the communities in the population over generations.

Secondly, GAs as an evolutionary computational approach have a unique advantage which makes us able to prioritize genes in the order of their relevance to disease genes. As discussed in Section 6.1, the idea is that the more frequently a gene is chosen to be in the optimizing communities, i.e. the more often a gene is selected for the more optimized communities of the higher generations, the more it is associated with the known disease genes, and therefore should have a higher score compared to others that are selected less often.

Thirdly, genes associated with a specific disease may act in separate communities which work with one another, or separate communities which overlap. We intuitively believe that GAs give our method the ability to give a high score to candidate disease genes closely working with the known disease genes whether they are in different communities or in overlapped communities for the following reasons. First, as the evolving populations contain thousands of different communities, different communities which work with the known disease genes would have the chance to evolve and be in the population at the same time. Genes in all such communities will get high scores as they are often selected in a number of the population's communities over generations, thereby they can increase their scores. Second, potential disease genes which lie in more than one community working with disease genes are selected more frequently as they can have the chance to be in many of the communities of the populations.

\subsection{Details of the Genetic Algorithm}

\subsubsection{Fitness Evaluation}

As previously stated, our GA tries to increase the degree of collaboration (i.e. the modularity) of the nodes (i.e. genes) inside the evolving communities. This is mainly because a community is recognized as a subgraph that is denser inside in comparison to the areas of the network around the subgraph. Therefore, as we can see in the community identification problem literature, measuring the modularity of a given community revolves around comparing the density of the area residing inside the community to the areas around the community. To see a general review of the various metrics used to measure the quality of a given community, see [81].

Hence, reasonably enough, the fitness value should assess the modularity degree 
of the candidate communities (individuals in a given generation) to increase the likelihood of reproduction for the more modular individuals across the generations.

For the experiments we have performed so far, we have used the simple modularity function $Q$ proposed by Luo et al. [57], also known as subgraph modularity. This modularity function simply divides the number of the edges inside the community $C$ by the number of all the edges involved with the community including the edges which are completely inside the community of $C$ and the edges connecting a node inside the community to a node outside the community:

$$
Q(C)=\sum_{i \in C} \frac{K_{i}^{\text {in }}(C)}{K_{i}(C)=K_{i}^{\text {in }}(C)+K_{i}^{\text {out }}(C)}
$$

where

$$
K_{i}^{i n}(C)=\sum_{j \in C} E(i, j)
$$

is the number of edges connecting $i$ to other nodes in $C$ and

$$
K_{i}^{\text {out }}(C)=\sum_{j \notin C} E(i, j)
$$

is the number of edges connecting $i$ to the nodes out of $C$.

This modularity function has been observed to be one of the most effective functions to evaluate the modularity degree of communities. For examples, Vasudevan et al [81] used this modularity function and successfully discovered communities in several real-world complex networks.

\subsubsection{Individuals (Chromosomes)}

As mentioned in Section 6.1, individuals (candidate solutions) are induced subnetworks that are potential disease gene communities. Individuals are represented using binary arrays of length $N$, where $N$ is the size of the protein-protein interaction network (i.e. the number of all the genes in the network). If the $i$ th element of the array is 1 , then the $i$ th gene of the network is considered to be in the candidate community; otherwise it is outside of the candidate community. 


\subsubsection{Linkage with known disease genes: Guilt by Associa- tion}

Like most of the computational disease gene prediction frameworks, this method also revolves around the Guilt by Association principle. This principle is crucial here to make sure that the final selected disease community contains the genes interacting with a high-degree of modularity (collaboration) to the known disease genes as well as to each other.

To provide this important feature for our GA-based method, all the individuals in all the populations (i.e. all the induced evolving subgraphs) always hold the known disease genes inside them. In fact, we force all evolving subnetworks to include all known disease genes. In other words, all the known disease genes are added to candidate communities while their fitness values (described in Section 6.3.1) are measured. This approach steers the whole process to finding communities in which genes are in high collaboration with the known disease genes.

\subsubsection{Maximum Community Size}

The maximum number of nodes (genes) that can be inside a candidate community (individual representing a sub-network) is determined in advance of starting the GA. As a matter of fact, we deliberately force all candidate communities to contain no more than a specific fraction of the network's genes. This is primarily for the following reasons: (1) to avoid obtaining unwanted futile solutions such as a big community containing most of the network's genes (which cannot be considered a community any more), (2) to provide a more competitive environment across the communities' evolution, and above all (3) to supervise the convergence trajectory of the sub-networks towards the disease genes community we wish to obtain, i.e. the way in which the individuals converge towards the community of disease genes over generations in order to define a scoring system to prioritize candidate genes (see Section 6.4.1).

In other words, candidate communities of the pre-determined maximum size could be interpreted as frames that move over the various areas of the network during the generations of the GA. Being evolved by the GA, these frames have a tendency to move towards the areas of the network that show higher collaborations and modularity with the known disease genes. As a matter of fact, candidate communities (individuals in the population of the GA) converge to areas of the network that are more likely to contain genes with a high level of collaboration with known disease genes.

To avoid acquiring biased output, we perform the same GA experiment several 
times to make sure that the population, i.e. all induced sub-networks, converges towards the same area of the network as the disease genes community.

\subsection{Prioritization of the Candidate Genes}

According to the disease gene prediction literature, there are two main approaches for disease-gene association [68]. The results obtained by a disease-gene association method could be either a prioritization of the candidate genes, i.e. a ranking of the candidate genes based on the likelihood of their involvement, or a selection of the candidate genes, i.e. a reduced set of candidate genes which have priority to be studied over the other candidates or that are more noteworthy in some way. Our computational approach is basically a selection-based and prioritization-based method, with the capability of offering a reduced set of candidate genes. Our GA-based approach ranks the candidate genes according to the new scoring system we devised, introduced in Section 6.4.1.

Furthermore, the communities with highest fitness value (modularity value) can be considered as the reduced set of candidate disease genes that is noteworthy for further studies.

\subsubsection{Scoring System}

Each experiment performed by our GA contains the following as input data:

- a set of known disease genes

- a relatively large PPI network containing all the genes existing in the known disease gene set

- a set of candidate genes containing all the other genes in the procured PPI network.

All the genes cumulatively gather scores while the candidate communities (individuals) are evolved over generations. The scoring method is based on the idea that genes which are more frequently selected in candidate communities, while they are being evolved over generations, should accumulate higher scores. Therefore, genes which survive for a greater number of generations, and which are in a greater number of communities, acquire higher scores. The cumulative score of a given gene is proportional to the fitness value of the communities for which it is selected. In other 
words, in being selected for better communities (i.e. individuals with higher fitness value), genes get relatively higher scores. Over generations, candidate communities (individuals of every generation) gradually converge into sets of genes that are in higher collaboration with the known disease genes and with one another. Therefore as the candidate communities are evolved by the GA, they are more likely to be in the vicinity of the community we are looking for, i.e. the predicted disease genes set.

The scoring function for a given candidate gene is calculated based upon Algorithm 1 , where $Q(c)$ is the modularity of the community $C$ as defined in Section 6.3.1.

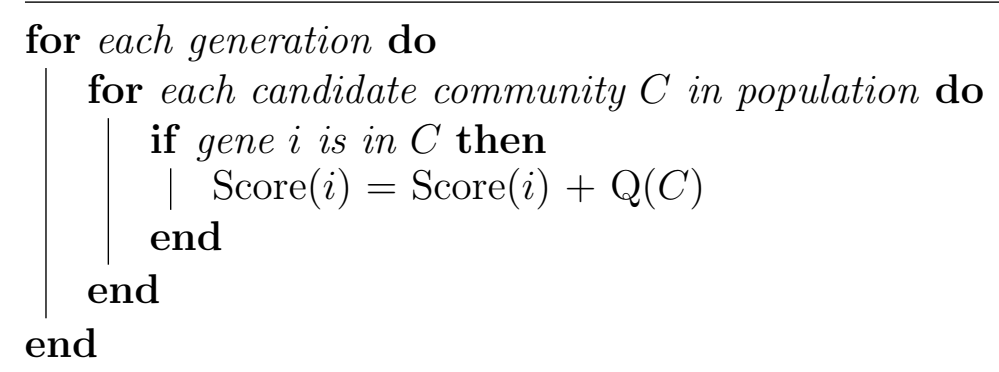

Algorithm 1: Calculate score of gene $i$ 


\section{Chapter 7}

\section{Datasets and Benchmark Tests}

\subsection{Databases}

\subsubsection{Protein-Protein Interaction Data}

We obtained the human PPI interaction network using GeneMANIA [84], reported to be among the best performing methods in a public large scale computational biology challenge [71]. The homo sapiens (human) interaction database of GeneMania recorded different types of interactions (co-expression, protein-protein interaction, pathway, co-localization etc.) based on 395 different interaction databases developed so far. It contains the interactions among 21435 genes from the human genome.

\subsubsection{Disease-Gene Association Data}

In order to select disease genes and make the association between particular diseases and particular genes, we used Genotator [82], developed at Harvard University. Genotator is a realtime aggregation tool that provides both comprehensive coverage and reliable gene-to-disease rankings for many of the diseases. It integrates data from 11 well-known frequently used clinical genetics resources including Pubmed [1], OMIM [40], GeneCards [74], HuGE Navigator [88], GenAtlas [30], WikiGenes [44] and some other phenotype-gene association data sources to rank genes in order of disease relevance. 


\subsection{Benchmark Test}

\subsubsection{Leave-One-Out Cross-Validation}

To assess the performance of our GA-based method, we used the leave-one-out crossvalidation procedure. This procedure is the most frequently used procedure to assess many of the state-of-the-art disease-gene associations approaches including [86], [80] and $[52]$.

According to leave-one-out cross-validation procedure, for every time performing the experiment, one of the known disease genes is removed from the known disease genes set, to observe if the method can again recover this gene as a high associative gene or not. In other words [55], given a set of known disease genes $D$ and a set of candidate genes $C$ a gene $d \in D$ is left out and all genes in $C \cup\{d\}$ are ranked using the computational approach under study. This process is repeated for every genes in the known disease genes set.

\subsubsection{Fold enrichment analysis}

Fold enrichment analysis is a concept one needs to know about for using the leave-oneout cross-validation procedure. Here, we offer the definition provided by X Wu et al [86] as it is much simpler and clearer than other definitions we could find: if a method successfully ranks known disease genes in the top $m \%$ of all candidate genes for $n \%$ of the known disease genes, there is a $n / m$-fold enrichment on average. Therefore, a threshold must be chosen to separate the two prediction classes. If the left-out known disease genes in leave-one-out cross-validation do not exceed the specified threshold in the candidate genes ranking, then it is considered as a successfully predicted gene in the validation.

\subsubsection{Receiver-Operating Characteristic (ROC) analysis}

Another concept involved with the leave-one-out cross-validation performance measure is the receiver-operating characteristic (ROC) analysis. ROC is defined as a plot to measure the performance of a binary classifier according to a defined discriminating threshold. Here, for the disease-gene association/prioritization problem, ROC plots the true-positive(TP) rate versus the false-positive(FP) rate, subject to the threshold separating the prediction classes (see Section 7.2.2). Consider the sets $D$ and $C$ and disease gene $d$ introduced in Section 7.2.1 again. Suppose $\theta$ is the threshold which the rank of known disease gene $d$ must not exceed in order to be considered as a 
successfully predicted known disease gene. TP(true positive) is the number of test cases where the ranking of $d$ is less than $\theta$, and $\mathrm{FN}$ (false negative) is the number of test cases where the ranking of $d$ exceeds $\theta$. The sensitivity of the computational disease-gene prioritization method for a given experiment is then defined as equation 7.1:

$$
\text { Sensitivity }=\frac{T P}{T P+F N}
$$




\section{Chapter 8}

\section{Case Studies and Results}

As a proof of concept, we applied our methodology to the problem of finding genes involved in breast cancer and Parkinson's disease. We selected these genetic disorders due to the diversity of related studies and because there is a significant amount of research literature.

To see a sample of output generated by our method see appendix A.

\subsection{Breast Cancer}

\subsubsection{Input Data}

We used the same input data as Xuebing et al [86] used to demonstrate the ability of CIPHER, one of the most well-known and frequently used disease-gene association framework. Using OMIM[40], they selected 16 disease genes known to be involved in breast cancer. They measured the ability of CIPHER to predict disease genes based on these 16 genes. Table 8.1 outlines these genes.

Using GeneMania (see 7.1.1), we obtained an un-weighted protein-protein interaction network containing the 16 disease genes shown in Table 8.1. We defined the candidate genes to be the first 2000 genes scored by GeneMania in the order of interactions with these 16 disease genes.

\subsubsection{GA Parameters}

We ran our GA-based method on this PPI network 20 separate times. Each run was on two threads and took between 5 to 10 hours. Table 8.2 outlines our GA parameters for all 20 executions of the GA. These parameters were empirically determined. It should 
Table 8.1: Known disease genes for breast cancer

\begin{tabular}{|c||c|}
\hline Gene & NCBI ID \\
\hline \hline BRCA1 & 672 \\
\hline BRCA2 & 675 \\
\hline TP53 & 7157 \\
\hline AR & 367 \\
\hline ATM & 472 \\
\hline CHEK2 & 11200 \\
\hline STK11 & 6794 \\
\hline RAD51 & 5888 \\
\hline PTEN & 5728 \\
\hline BARD1 & 580 \\
\hline RB1CC1 & 9821 \\
\hline NCOA3 & 8202 \\
\hline PIK3CA & 5290 \\
\hline PPM1D & 8493 \\
\hline CASP8 & 841 \\
\hline TGF1 & 281527 \\
\hline
\end{tabular}

be noted that further study should be performed on determining the most appropriate values for these parameters for other experiments (e.g. for other diseases).

Table 8.2: GA Parameter Settings for breast cancer experiment

\begin{tabular}{|c||c|}
\hline \multicolumn{1}{|c||}{ Parameter } & Value \\
\hline \hline number of generations & 300 \\
\hline population size & 5000 \\
\hline cross-over rate & $95 \%$ \\
\hline mutation rate & $5 \%$ \\
\hline tournament size & 3 \\
\hline fitness evaluation & $Q(C)($ eq 6.1$)$ \\
\hline maximum community size & 50 \\
\hline
\end{tabular}

Figure 8.1 depicts the progression of the averages of best fitness values, and the averages of average fitness values for the 20 runs of the GA over the generations.

A sample of generated candidate genes ranking regarding this experiment is provided in Appendix B.1. The community with the highest modularity (Q - equation 6.1) procured by this experiment is also provided in Appendix C.1. 


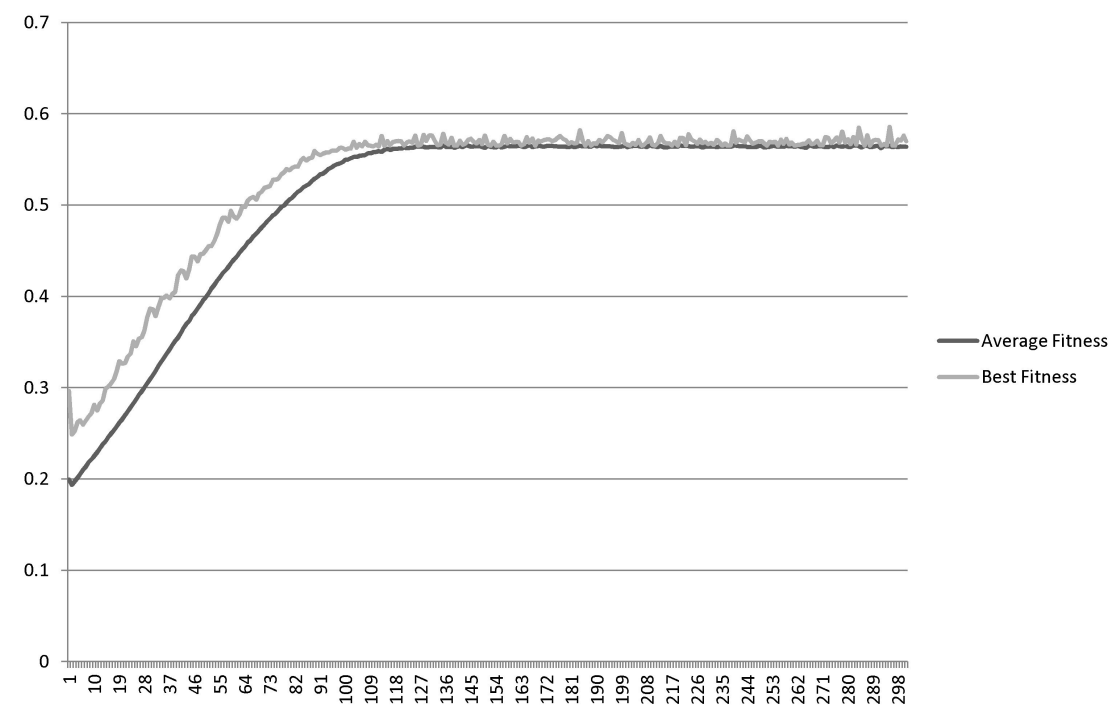

Figure 8.1: averages of best/average fitness values over generations (breast cancer experiment)

\subsection{Analysis}

\subsubsection{Leave-One-Out Cross-Validation}

Running our GA-based method on the input data described in Section 8.1.1 and based on the parameters outlined in Table 8.2, and using leave-one-out cross-validation method (explained in 7.2.1), our method successfully predicted 12 out of 16 known genes as being involved in breast cancer. The threshold ( $\theta$ - see Section 7.2) was set to the size of the community of the disease-genes the GA was evolving over generations (here, 50). This is discussed further in chapter 9. Table 8.3 shows the 12 predicted genes and median of their 20 different rankings as a result of running the experiment 20 times.

\subsubsection{Comparison with CIPHER}

Based on the results CIPHER gained over the same known genes provided in [86], CIPHER successfully ranked known disease genes in the top $0.917 \%$ of all candidate genes in $49.1 \%$ of test cases, giving CIPHER a fold enrichment of 53.50 on average. Our GA-based approach, if we consider the top 1\% of all candidate genes, gives fold 
Table 8.3: 12 successfully predicted genes out of the 16 known disease genes for breast cancer listed in Table 8.1

\begin{tabular}{|c||c||c||c|}
\hline Gene & Median of rankings & Best ranking & Standard Deviation of rankings \\
\hline \hline BRCA1 & 1 & 1 & 1.47 \\
\hline TP53 & 1 & 1 & 1.15 \\
\hline BRCA2 & 5 & 1 & 2.33 \\
\hline ATM & 6 & 3 & 1.89 \\
\hline AR & 8 & 2 & 3.24 \\
\hline PPM1D & 16.50 & 8 & 4.49 \\
\hline BARD1 & 19.50 & 5 & 6.04 \\
\hline RAD51 & 20 & 13 & 9.67 \\
\hline RB1CC1 & 22 & 10 & 11.22 \\
\hline PTEN & 34.50 & 22 & 12.21 \\
\hline STK11 & 42.50 & 17 & 17.08 \\
\hline NCOA3 & 46 & 27 & 16.71 \\
\hline
\end{tabular}

enrichment of 50 on average, fold enrichment of 56.25 for the ranking offered by the best solution procured by the GA, and fold enrichment of 62.50 if we consider the best rankings of the known disease genes among all 20 rankings given by the GA.

Based on the acquired results, ROC analysis (see Section 7.2.3) gives us 12 TPs and 4 FNs, thus the sensitivity of this experiment is $75 \%$. The sensitivity of the experiment for CIPHER was $62.50 \%$ as a result of 10 TPs and 6 FNs.

\subsubsection{Comparison with Random Walk}

We briefly discussed random walk methods in Section 4.2. Random walk methods have been observed to outperform other types of disease-gene association approaches discussed in Chapter 4 ([62],[9], [9]).

We performed this experiment over the same known disease genes and candidate genes using GPEC [55], disease gene prioritization and prediction framework based on RWRA (random walk with restart algorithm - see Section 4.2).

Applying the same threshold $(\theta=50)$, GPEC gives sensitivity of 0.8112 (13 TPs) and fold enrichment of 56.25 for the top $1 \%$ of all candidate genes. 


\subsubsection{Summary of the Analysis for Breast Cancer Experi- ment}

Table 8.4 summarizes the analysis described in Sections 8.2.1 to 8.2.3. See Section 7.2 for description of fold enrichment, leave-one-out and sensitivity measurements.

Table 8.4: Analysis and comparison for breast cancer experiment

\begin{tabular}{|c||c||c||c|}
\hline Method/Framework & Fold enrichment & Leave-one-out & sensitivity \\
\hline \hline CIPHER & 53.50 & $10 / 16$ & 0.6250 \\
\hline GPEC(random walk) & 56.25 & $13 / 16$ & 0.8112 \\
\hline our method (median ranking) & 50 & $12 / 16$ & 0.75 \\
\hline our method (best ranking) & 56.25 & $12 / 16$ & 0.75 \\
\hline
\end{tabular}

As table 8.4 suggests, our method outperforms CIPHER for this experiment and the results obtained by our method are comparable to the results acquired by random walk.

\subsubsection{Predicting new Disease Genes}

We briefly studied other candidate genes ranked among the top $1 \%$ of the candidate genes on average using Genotator (see Section 7.1.2). Our GA successfully predicted some other genes involved in breast cancer which were not in the known disease-genes set of the performed experiment. Among the other potential breast cancer genes ranked among the top 1\% are ESR1, NBN, CHECK1, H2AFX ,EP300, SMAD3, and other genes most of which have evidence of being involved in breast cancer by different data sources according to Genotator.

\subsection{Parkinson's Disease}

\subsubsection{Input Data}

For Parkinson's disease, we first extracted 15 genes known to be involved in this disease from Genotator. Table 8.5 lists these 15 genes. These are the top 15 genes scored by Genotator in terms of relevance to Parkinson's Disease. Although some of these genes lie on different loci (chromosomes) of the DNA, the functional proteins 
derived from these genes must physically interact with one another across the physical interaction network (PPI) containing these genes.

Table 8.5: known disease genes for Parkinson Disease

\begin{tabular}{|c||c|}
\hline Gene & NCBI ID \\
\hline \hline LRRK2 & 120892 \\
\hline SNCA & 6622 \\
\hline PARK2 & 5071 \\
\hline MAPT & 4137 \\
\hline APOE & 348 \\
\hline GBA & 2629 \\
\hline GAK & 2580 \\
\hline BST1 & 683 \\
\hline DRD2 & 1813 \\
\hline PINK1 & 65018 \\
\hline MAOB & 4129 \\
\hline BDNF & 627 \\
\hline CYP2D6 & 1565 \\
\hline PON1 & 5444 \\
\hline COMPT & 1312 \\
\hline
\end{tabular}

Using GeneMania, we obtained an un-weighted physical interaction network (proteinprotein interaction network) containing the 15 known disease genes. We defined the candidate genes to be the first 3000 genes scored by GeneMania in the order of interactions with these 15 disease genes.

\subsubsection{GA Parameters}

Like the previous experiments, we ran our GA 20 separate times (see Section 8.1.2). Each run was on two threads and tool 5 to 7 days. Table 8.6 outlines the parameters for this experiment. These parameters were empirically determined. It should be noted that further study should be performed on determining the most appropriate values for these parameters for other experiments (e.g. for other diseases). Figure 8.2 shows averages of best/average fitness values over generations.

A sample of generated candidate genes ranking regarding this experiment is provided in Appendix B.2. The community with the highest modularity (Q - Equation 6.1) procured by this experiment is also provided in Appendix C.2. 
Table 8.6: GA Parameter Settings for Parkinson Disease experiment

\begin{tabular}{|c||c|}
\hline \multicolumn{1}{|c||}{ Parameter } & Value \\
\hline \hline number of generations & 500 \\
\hline population size & 30,000 \\
\hline cross-over rate & $95 \%$ \\
\hline mutation rate & $5 \%$ \\
\hline tournament size & 4 \\
\hline fitness evaluation & $Q(C)($ eq 6.1$)$ \\
\hline maximum community size & 100 \\
\hline
\end{tabular}

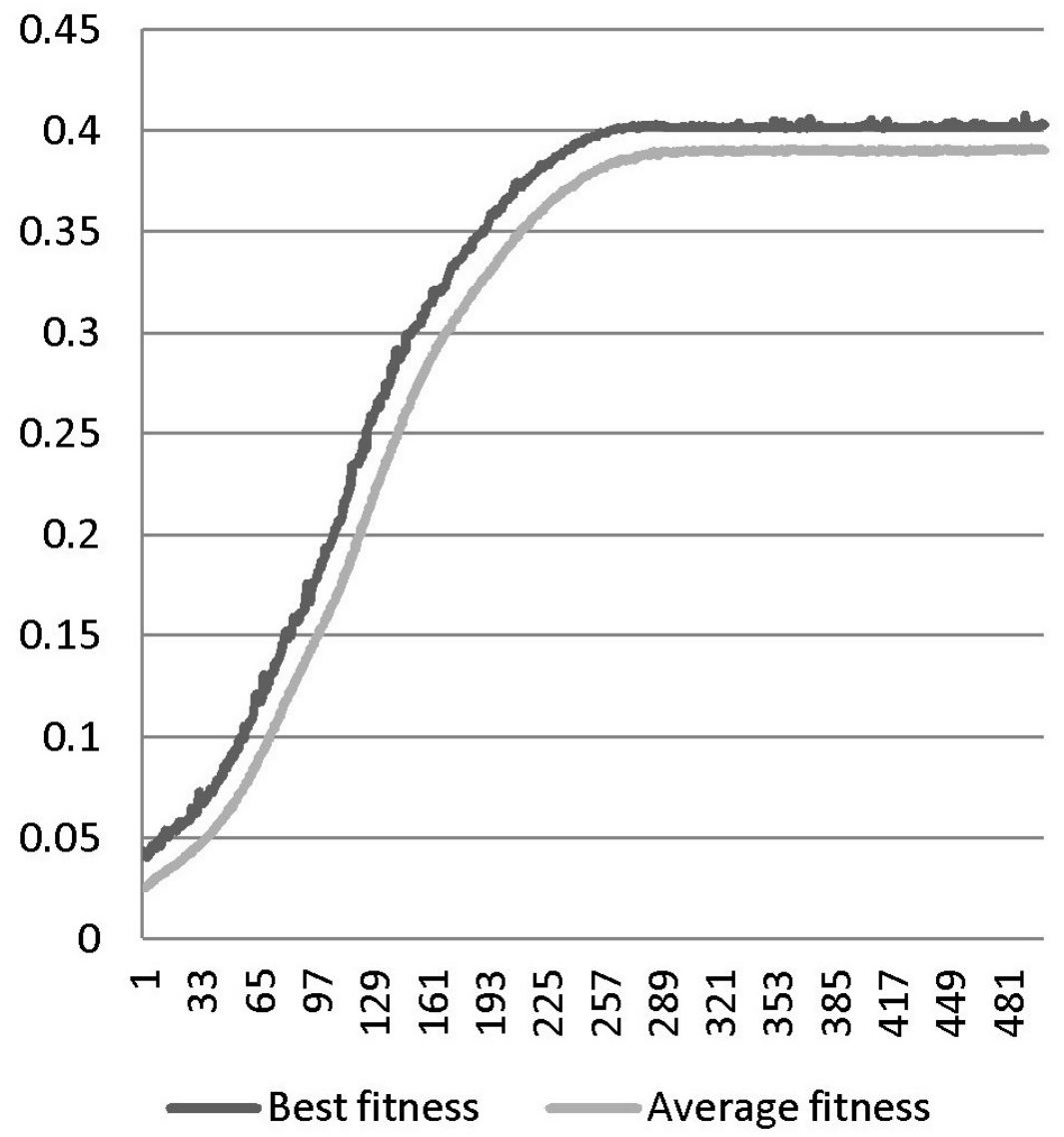

Figure 8.2: Averages of best/average fitness values over generations

\subsubsection{Leave-One-Out Cross-Validation}

For this experiment, our method successfully predicts 9 out of 15 genes in leave-oneout cross-validation on its average performance among 20 executions. Similar to the previous experiment, the threshold was set to the maximum community size (in this 
case 100). Table 8.7 summarizes the results.

Table 8.7: 9 Successfully predicted genes out of the 15 known disease genes for Parkinson's Disease listed in Table 8.5

\begin{tabular}{|c||c||c||c|}
\hline Gene & Median of rankings & Best Ranking & Standard Deviation of rankings \\
\hline \hline SNCA & 1 & 1 & 2.26 \\
\hline PARK2 & 10 & 3 & 3.06 \\
\hline APOE & 18.50 & 8 & 6.23 \\
\hline MAPT & 38.50 & 23 & 9.23 \\
\hline PINK1 & 46 & 14 & 10.19 \\
\hline DRD2 & 63.50 & 38 & 8.63 \\
\hline BDNF & 71 & 38 & 18.75 \\
\hline GAK & 73.50 & 33 & 19.44 \\
\hline LRRK2 & 92.50 & 66 & 21.17 \\
\hline
\end{tabular}

\subsubsection{Comparison with ENDEAVOUR and Random Walk (GPEC)}

We performed this experiment with ENDEAVOUR [4], one of the most well-known and frequently used frameworks for disease gene prioritization, on the same known disease genes and candidate disease genes set for Parkinson's disease. We also performed this experiment with GPEC which we introduced in Section 8.2.3. Table 8.8 outlines the results. See Section 7.2 for description of fold enrichment, leave-one-out and sensitivity measurements.

Table 8.8: Analysis and comparison for Parkinson's disease experiment

\begin{tabular}{|c||c||c||c|}
\hline Framework & Fold enrichment(top 1\%) & Leave-one-out & sensitivity \\
\hline \hline ENDEAVOUR & 26.06 & $7 / 15$ & 0.46 \\
\hline GPEC(random walk) & 40 & $8 / 15$ & 0.53 \\
\hline our method (median) & 33.33 & $9 / 15$ & 0.60 \\
\hline our method (best) & 40 & $9 / 15$ & 0.60 \\
\hline
\end{tabular}




\subsubsection{Predicting novel Parkinson's disease genes}

Amongst the candidate genes frequently ranked in the top 10 genes in several runs of the experiments, there are some genes which can evidentially be associated with Parkinson's Disease. Interestingly, for some genes ranked among the top places the supporting evidence and studies are fairly recent.

\section{Epidermal Growth Factor Receptor (EGFR)}

According to [24], the protein encoded by this gene is a receptor for members of the epidermal growth factor family. EGFR is a cell surface protein that binds to epidermal growth factor. Increased activities of REGF or mutations in REFG have been observed in certain types of cancers [24]. Our computational method suggested that EGFR is one of the highest-scoring genes that closely interacts with the 15 known Parkinsons disease genes used in this experiment. In quite a few recent studies, epidermal growth factor has been suggested as a candidate gene for cognitive impairment in Parkinsons disease [67]. Chen-Plotkin et al. [15] and Pellecchia et al. [67] have recently observed links between epidermal growth factor and cognitive inabilities of the Parkinsons disease patients.

\section{ABL1 gene}

The ABL1 gene (gene ID: 25 see [25]) was also one of the top 10 genes in most of the 20 rankings obtained by our method. In a very recent study performed by Hebron et al. [43], it was observed that a level of ABL1 activity is increased in human postmortem Parkinson's Disease brains. Mahul-Mellier et al. [58] is another very recent study indicating links between the c-Abl protein derived from the ABL1 gene and Parkinson's Disease.

\section{Ubiquitin C (UBC) gene}

UBC [26] was also frequently ranked among the very top genes in our experiment. Mutations in UBC have been repeatedly observed to be responsible for Parkinsons disease [18]. For instance, in a very recent research performed by Viorica Raluca Contu et al. [18], the roles of mutation in ubiquitin C-terminal hydrolase L1 (UCHL1) have been studied. PubMed has recorded remarkable number of publications discussing the UBC's role in Parkinsons disease. 


\subsection{Analysing GA capability to evolve disease gene communities with higher modularity}

To analyse the ability of GA to evolve disease gene communities with higher modularity, we hereby compare the results obtained by the experiment we performed for breast cancer (Section 8.1) with the results acquired by a random search done by GA. To simulate a random search using GA, we set the tournament size to 1 and ran GA for 20 times on the same candidate and known disease genes sets.

Figure 8.3 depicts fitness growth (average of average and best fitness values for 20 runs) over generations while the search is random (tournament size is 1) and while the search is not random (tournament size is 3 ).

We performed a t-test on average and best fitness values generated by the two approaches explained above. The very low p-values suggest that the results acquired by our experiments are significantly different from the results given by the random approach. See Table 8.9 for more details.

We ran our GA 20 different times with the tournament selection of 1 and pick the best possible ranking out of the 20 generated rankings. We subsequently compared this ranking with the average ranking acquired by the experiment we performed for breast cancer explained in Section 8.1. Table 8.10 briefly outlines this comparison. The very low p-values (close to 0) suggests that the difference is obviously significant. See Section 7.2 for description of fold enrichment, leave-one-out and sensitivity measurements.

Table 8.9: P-values given by t-test

\begin{tabular}{|c||c|}
\hline & P-Value \\
\hline \hline Comparing Average Fitness Values & $7.0602 \mathrm{E}-278$ \\
\hline Comparing Best Fitness Values & 0 \\
\hline
\end{tabular}




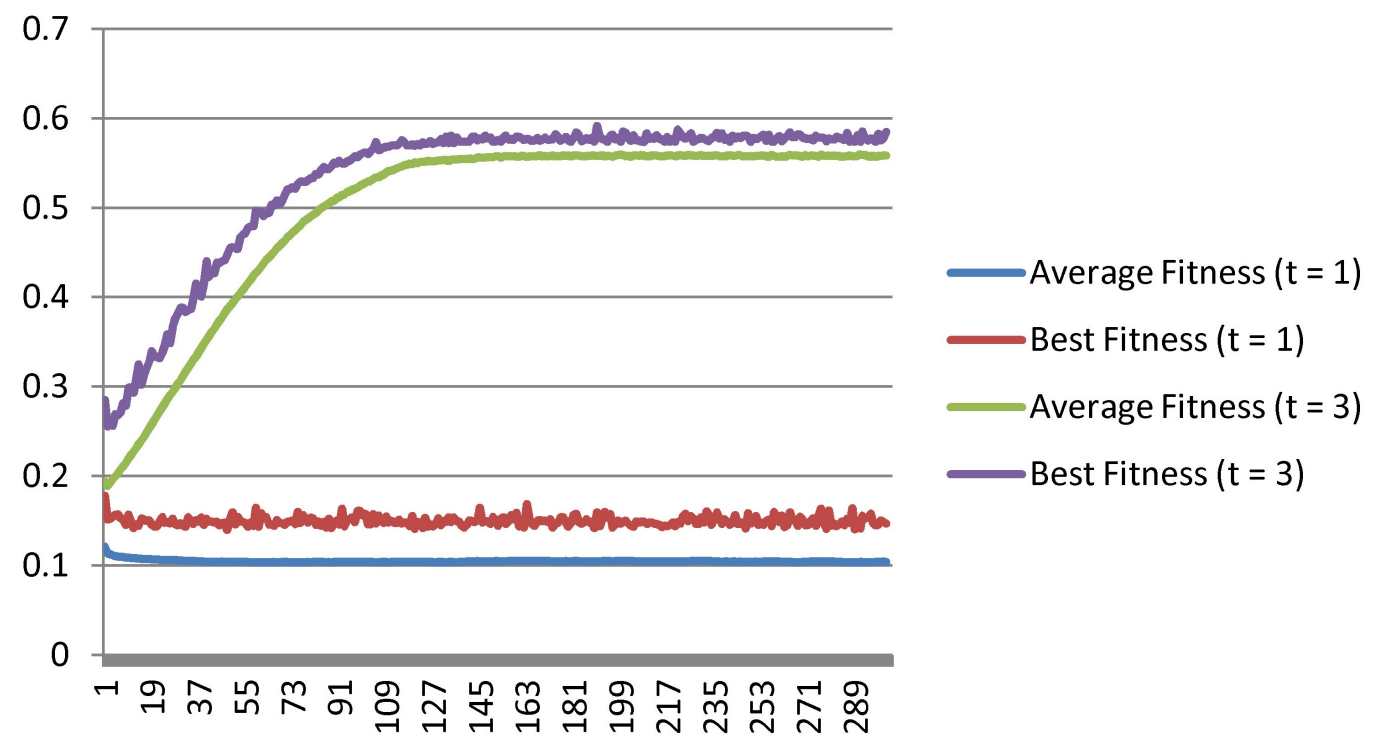

Figure 8.3: comparing averages of best/average fitness values over generations while $\mathrm{t}=1$ and while $\mathrm{t}=3$ )

Table 8.10: Comparing results for tournament size of 1 and 3

\begin{tabular}{|c||c||c|}
\hline Tournament Size & Fold enrichment(top 1\%) & Leave-one-out (sensitivity) \\
\hline \hline 3 & 50 & 0.625 \\
\hline 1 & 0.062 & 0.062 \\
\hline
\end{tabular}




\section{Chapter 9}

\section{Conclusions and Future Work}

In this study, we demonstrated a new computational disease-gene association method with the use of a genetic algorithm. As elaborated earlier, our method tries to evolve different communities of candidate genes with as much collaboration with the whole set of known disease genes as possible. It starts from randomly created communities of genes and optimizes the communities over generations, based on the modularity of the community (according to equation 6.1).

The main idea behind the scoring system (described in algorithm 1) is that the more frequently a gene is chosen to be in the optimizing communities, i.e. the more a gene is selected for the more optimized communities of the higher generations, the more it is associated with the known disease genes, and therefore should have a higher score compared to others that are selected less often.

Genes associated with a specific disease may act in separate communities which work with one another, or separate communities which overlap. We intuitively believe that our GA-based computation is capable of finding disease genes working in different communities or in overlapped communities for the following reasons. First, as the evolving populations contain thousands of different communities, different communities which work with the known disease genes would have the chance to evolve and be in the population at the same time. Genes in all such communities will get high scores as they are often selected in a number of the population's communities over generations, thereby they can increase their scores. Secondly, potential disease genes which lie in more than one community working with disease genes are selected more frequently as they can have the chance to be in many of the communities of the populations.

As a proof of concept, we first tested our approach on 16 breast cancer genes taken from similar experiments performed by Xuebing et al [86] to test the performance 
of CIPHER, a well-known disease gene prioritization framework (see Section 8.1). We also tested our method on 15 Parkinson's Disease genes taken from GeneMania [84]. We compared our results to the results gained by CIPHER, ENDEAVOUR and GPEC, three of the most reliable and frequently used disease-gene ranking frameworks. The results are satisfactory as two of the first experiments using a newly developing approach (see chapter 8), however, there should be specific and more accurate strategies to determine the known disease gene set with regard to different diseases and choosing appropriate GA parameters.

Determining an appropriate maximum community size for use in our method is also a matter of discussion. Different maximum community sizes can obviously affect the number of times each gene would be allowed inside the evolving community, thereby affecting the scores acquired by each gene. Here we chose a maximum community of size of 50 and 100 for the breast cancer and Parkinson's disease respectively, however further investigation should be conducted on this parameter, considering supporting reasons for choosing a given value. The accuracy of the results may possibly be increased as a result. Concisely speaking, appropriate strategies and different parameter values may vary significantly from one disease to another.

Although protein-protein interaction networks have been shown to be among the most powerful pieces of evidence for disease-gene association, there are still major concerns about the amount, accuracy, and quality of the available data, and there are still a considerable amount of interactions that are not well-studied. To obtain more accurate and reliable disease genes communities one may use a fusion of different network-based data resources along with PPI (see Section 3.3). The possibility of adding input data regarding other evidence discussed in chapter 3 has been provided for our method. Further studies can assess the performance of our method working on other interaction networks such as functional annotation, co-expression network, and regulatory networks.

It should be noted nonetheless that this is a proof of concept and that much further study remains. Most importantly, this method should be applied to other diseases. Also, as specified above, future work must include further study of appropriate parameters, especially with respect to appropriate parameters for different diseases.

Finally, the genes in the communities with highest fitness values (modularity value) generated by our GA, and also the genes ranked as top genes in the rankings are noteworthy for further studies from a biological perspective. 


\section{Bibliography}

[1] Abigail Acland, Richa Agarwala, Tanya Barrett, Jeff Beck, Dennis A Benson, Colleen Bollin, Evan Bolton, Stephen H Bryant, Kathi Canese, Deanna M Church, et al. Database resources of the national center for biotechnology information. Nucleic acids research, 42(D1):D7, 2014.

[2] Balázs Adamcsek, Gergely Palla, Illés J Farkas, Imre Derényi, and Tamás Vicsek. Cfinder: locating cliques and overlapping modules in biological networks. Bioinformatics, 22(8):1021-1023, 2006.

[3] Euan A Adie, Richard R Adams, Kathryn L Evans, David J Porteous, and Ben S Pickard. Speeding disease gene discovery by sequence based candidate prioritization. BMC bioinformatics, 6(1):55, 2005.

[4] Stein Aerts, Diether Lambrechts, Sunit Maity, Peter Van Loo, Bert Coessens, Frederik De Smet, Leon-Charles Tranchevent, Bart De Moor, Peter Marynen, Bassem Hassan, et al. Gene prioritization through genomic data fusion. Nature biotechnology, 24(5):537-544, 2006.

[5] Russ B Altman, Casey M Bergman, Judith Blake, Christian Blaschke, Aaron Cohen, Frank Gannon, Les Grivell, Udo Hahn, William Hersh, Lynette Hirschman, et al. Text mining for biology-the way forward: opinions from leading scientists. Genome Biol, 9(Suppl 2):S7, 2008.

[6] ArneLH. Gene expression control. http://commons.wikimedia. org/wiki/File:Gene_expression_control.png\#mediaviewer/File: Gene_expression_control.png, 2014. [Online; accessed 30-Sep-2014].

[7] Michael Ashburner, Catherine A Ball, Judith A Blake, David Botstein, Heather Butler, J Michael Cherry, Allan P Davis, Kara Dolinski, Selina S Dwight, Janan T Eppig, et al. Gene ontology: tool for the unification of biology. Nature genetics, 25(1):25-29, 2000. 
[8] Gary D Bader and Christopher WV Hogue. An automated method for finding molecular complexes in large protein interaction networks. BMC bioinformatics, $4(1): 2,2003$.

[9] Albert-László Barabási, Natali Gulbahce, and Joseph Loscalzo. Network medicine: a network-based approach to human disease. Nature Reviews Genetics, 12(1):56-68, 2011.

[10] Christian Blaschke, Miguel A Andrade, Christos A Ouzounis, and Alfonso Valencia. Automatic extraction of biological information from scientific text: proteinprotein interactions. In Ismb, volume 7, pages 60-67, 1999.

[11] Yana Bromberg. Disease gene prioritization. PLoS computational biology, 9(4):e1002902, 2013.

[12] Dongbo Bu, Yi Zhao, Lun Cai, Hong Xue, Xiaopeng Zhu, Hongchao Lu, Jingfen Zhang, Shiwei Sun, Lunjiang Ling, Nan Zhang, et al. Topological structure analysis of the protein-protein interaction network in budding yeast. Nucleic acids research, 31(9):2443-2450, 2003.

[13] Guido Caldarelli and Alessandro Vespignani. (edited by) Large scale structure and dynamics of complex networks: from information technology to finance and natural science, volume 2. World Scientific, 2007.

[14] Jing Chen, Bruce J Aronow, and Anil G Jegga. Disease candidate gene identification and prioritization using protein interaction networks. BMC bioinformatics, 10(1):73, 2009.

[15] Alice S Chen-Plotkin, William T Hu, Andrew Siderowf, Daniel Weintraub, Rachel Goldmann Gross, Howard I Hurtig, Sharon X Xie, Steven E Arnold, Murray Grossman, Christopher M Clark, et al. Plasma epidermal growth factor levels predict cognitive decline in parkinson's disease. Annals of neurology, 69(4):655-663, 2011.

[16] Caroline St Clair and Jonathan E Visick. Exploring Bioinformatics: A ProjectBased Approach. Jones \& Bartlett Publishers, 2011.

[17] Wikimedia Commons. Comparison of a single-stranded rna and a doublestranded dna with their corresponding nucleobases. http://commons. wikimedia.org/wiki/File:Difference_DNA_RNA-EN.svg, 2014. [Online; accessed 30-Sep-2014]. 
[18] Viorica Raluca Contu, Yaichiro Kotake, Takashi Toyama, Katsuhiro Okuda, Masatsugu Miyara, Shuichiro Sakamoto, Shigeyoshi Samizo, Seigo Sanoh, Yoshito Kumagai, and Shigeru Ohta. Endogenous neurotoxic dopamine derivative covalently binds to parkinson's disease-associated ubiquitin c-terminal hydrolase 11 (uch-11) and alters its structure and function. Journal of neurochemistry, 2014.

[19] Imre Derényi, Gergely Palla, and Tamás Vicsek. Clique percolation in random networks. Physical review letters, 94(16):160202, 2005.

[20] Zoltán Dezső, Yuri Nikolsky, Tatiana Nikolskaya, Jeremy Miller, David Cherba, Craig Webb, and Andrej Bugrim. Identifying disease-specific genes based on their topological significance in protein networks. BMC systems biology, 3(1):36, 2009.

[21] Stijn Dongen. A new cluster algorithm for graphs. 1998.

[22] Anton J Enright, Stijn Van Dongen, and Christos A Ouzounis. An efficient algorithm for large-scale detection of protein families. Nucleic acids research, 30(7):1575-1584, 2002.

[23] W Gregory Feero, Alan E Guttmacher, and Teri A Manolio. Genomewide association studies and assessment of the risk of disease. New England Journal of Medicine, 363(2):166-176, 2010.

[24] National Centre for Biotechnology Information. http://www.ncbi.nlm.nih.gov/gene/1956, May 2014.

[25] National Centre for Biotechnology Information. http://www.ncbi.nlm.nih.gov/gene/25, May 2014.

[26] National Centre for Biotechnology Information. http://www.ncbi.nlm.nih.gov/gene/7316, May 2014.

[27] Santo Fortunato. Community detection in graphs. Physics Reports, 486(3):75174, 2010.

[28] Santo Fortunato and Claudio Castellano. Community structure in graphs. In Computational Complexity, pages 490-512. Springer, 2012. 
[29] Jan Freudenberg and P Propping. A similarity-based method for genome-wide prediction of disease-relevant human genes. Bioinformatics, 18(suppl 2):S110S115, 2002.

[30] Jean Frézal. Genatlas database, genes and development defects. Comptes Rendus de l'Académie des Sciences-Series III-Sciences de la Vie, 321(10):805-817, 1998.

[31] Kyle J Gaulton, Karen L Mohlke, and Todd J Vision. A computational system to select candidate genes for complex human traits. Bioinformatics, 23(9):11321140, 2007.

[32] Michelle Girvan and Mark EJ Newman. Community structure in social and biological networks. Proceedings of the National Academy of Sciences, 99(12):78217826, 2002.

[33] Shuba Gopal, Anne Haake, Rhys Price Jones, and Paul Tymann. Bioinformatics: a computing perspective. McGraw-Hill Science/Engineering/Math, 2008.

[34] John Gribbin, Jeremy Cherfas, Douglas Palmer, and Adam Hart-Davis. Science: the definitive visual guide. Dorling Kindersley Ltd, 2009.

[35] Anthony JF Griffiths. An introduction to genetic analysis. Macmillan, 2005.

[36] Roger Guimera and Luis A Nunes Amaral. Functional cartography of complex metabolic networks. Nature, 433(7028):895-900, 2005.

[37] Emre Guney and Baldo Oliva. Exploiting protein-protein interaction networks for genome-wide disease-gene prioritization. PloS one, 7(9):e43557, 2012.

[38] David Hall. A single nucleotide polymorphism is a change of a nucleotide at a single base-pair location on dna. created using inkscape v0.45.1. http:// commons . wikimedia.org/wiki/File:Dna-SNP.svg, 2007. [Online; accessed 30Sep-2014].

[39] Ada Hamosh, Alan F Scott, Joanna S Amberger, Carol A Bocchini, and Victor A McKusick. Online mendelian inheritance in man (omim), a knowledgebase of human genes and genetic disorders. Nucleic acids research, 33(suppl 1):D514D517, 2005.

[40] Ada Hamosh, Alan F Scott, Joanna S Amberger, Carol A Bocchini, and Victor A McKusick. Online mendelian inheritance in man (omim), a knowledgebase of 
human genes and genetic disorders. Nucleic acids research, 33(suppl 1):D514D517, 2005.

[41] Jing-Dong J Han, Nicolas Bertin, Tong Hao, Debra S Goldberg, Gabriel F Berriz, Lan V Zhang, Denis Dupuy, Albertha JM Walhout, Michael E Cusick, Frederick P Roth, et al. Evidence for dynamically organized modularity in the yeast protein-protein interaction network. Nature, 430(6995):88-93, 2004.

[42] Leland H Hartwell, John J Hopfield, Stanislas Leibler, and Andrew W Murray. From molecular to modular cell biology. Nature, 402:C47-C52, 1999.

[43] Michaeline L Hebron, Irina Lonskaya, and CE Moussa. Tyrosine kinase inhibition facilitates autophagic snca/alpha-synuclein clearance. Autophagy, 9(8):12491250, 2013.

[44] Robert Hoffmann. A wiki for the life sciences where authorship matters. Nature genetics, 40(9):1047-1051, 2008.

[45] Trey Ideker and Roded Sharan. Protein networks in disease. Genome research, 18(4):644-652, 2008.

[46] Lars J Jensen, Michael Kuhn, Manuel Stark, Samuel Chaffron, Chris Creevey, Jean Muller, Tobias Doerks, Philippe Julien, Alexander Roth, Milan Simonovic, et al. String 8a global view on proteins and their functional interactions in 630 organisms. Nucleic acids research, 37(suppl 1):D412-D416, 2009.

[47] Jieun Jeong and Jake Y Chen. Techniques for prioritization of candidate disease genes. Computational Intelligence and Pattern Analysis in Biology Informatics, 13:309, 2010.

[48] Pall F Jonsson and Paul A Bates. Global topological features of cancer proteins in the human interactome. Bioinformatics, 22(18):2291-2297, 2006.

[49] Minoru Kanehisa, Michihiro Araki, Susumu Goto, Masahiro Hattori, Mika Hirakawa, Masumi Itoh, Toshiaki Katayama, Shuichi Kawashima, Shujiro Okuda, Toshiaki Tokimatsu, et al. Kegg for linking genomes to life and the environment. Nucleic acids research, 36(suppl 1):D480-D484, 2008.

[50] Maricel G Kann. Protein interactions and disease: computational approaches to uncover the etiology of diseases. Briefings in bioinformatics, 8(5):333-346, 2007. 
[51] Philip M Kim, Jan O Korbel, and Mark B Gerstein. Positive selection at the protein network periphery: evaluation in terms of structural constraints and cellular context. Proceedings of the National Academy of Sciences, 104(51):20274-20279, 2007.

[52] Sebastian Köhler, Sebastian Bauer, Denise Horn, and Peter N Robinson. Walking the interactome for prioritization of candidate disease genes. The American Journal of Human Genetics, 82(4):949-958, 2008.

[53] Andrea Lancichinetti, Filippo Radicchi, and José J Ramasco. Statistical significance of communities in networks. Physical Review E, 81(4):046110, 2010.

[54] Jonas B Laurila, Nona Naderi, René Witte, Alexandre Riazanov, Alexandre Kouznetsov, and Christopher JO Baker. Algorithms and semantic infrastructure for mutation impact extraction and grounding. BMC genomics, 11(Suppl 4):S24, 2010.

[55] Duc-Hau Le and Yung-Keun Kwon. Gpec: a cytoscape plug-in for random walk-based gene prioritization and biomedical evidence collection. Computational biology and chemistry, 37:17-23, 2012.

[56] Núria López-Bigas and Christos A Ouzounis. Genome-wide identification of genes likely to be involved in human genetic disease. Nucleic acids research, 32(10):3108-3114, 2004.

[57] Feng Luo, James Z Wang, and Eric Promislow. Exploring local community structures in large networks. Web Intelligence and Agent Systems, 6(4):387-400, 2008.

[58] Anne-Laure Mahul-Mellier, Bruno Fauvet, Amanda Gysbers, Igor Dikiy, Abid Oueslati, Sandrine Georgeon, Allan J Lamontanara, Alejandro Bisquertt, David Eliezer, Eliezer Masliah, et al. c-abl phosphorylates $\alpha$-synuclein and regulates its degradation: implication for $\alpha$-synuclein clearance and contribution to the pathogenesis of parkinson's disease. Human molecular genetics, 23(11):2858$2879,2014$.

[59] Marco Masseroli, Osvaldo Galati, and Francesco Pinciroli. Gfinder: genetic disease and phenotype location statistical analysis and mining of dynamically annotated gene lists. Nucleic acids research, 33(suppl 2):W717-W723, 2005. 
[60] Melanie Mitchell. Complexity: A guided tour. Oxford University Press, 2009.

[61] Hossein Najmabadi, Hao Hu, Masoud Garshasbi, Tomasz Zemojtel, Seyedeh Sedigheh Abedini, Wei Chen, Masoumeh Hosseini, Farkhondeh Behjati, Stefan Haas, Payman Jamali, et al. Deep sequencing reveals 50 novel genes for recessive cognitive disorders. Nature, 478(7367):57-63, 2011.

[62] Saket Navlakha and Carl Kingsford. The power of protein interaction networks for associating genes with diseases. Bioinformatics, 26(8):1057-1063, 2010.

[63] Saket Navlakha, Michael C Schatz, and Carl Kingsford. Revealing biological modules via graph summarization. Journal of Computational Biology, 16(2):253264, 2009.

[64] Martin Oti and Han G Brunner. The modular nature of genetic diseases. Clinical genetics, 71(1):1-11, 2007.

[65] Martin Oti, Berend Snel, Martijn A Huynen, and Han G Brunner. Predicting disease genes using protein-protein interactions. Journal of medical genetics, 43(8):691-698, 2006.

[66] Arzucan Özgür, Thuy Vu, Güneş Erkan, and Dragomir R Radev. Identifying gene-disease associations using centrality on a literature mined gene-interaction network. Bioinformatics, 24(13):i277-i285, 2008.

[67] Maria Teresa Pellecchia, Gabriella Santangelo, Marina Picillo, Rosario Pivonello, Katia Longo, Claudia Pivonello, Carmine Vitale, Marianna Amboni, Anna De Rosa, Marcello Moccia, et al. Serum epidermal growth factor predicts cognitive functions in early, drug-naive parkinsons disease patients. Journal of neurology, 260(2):438-444, 2013.

[68] Rosario M Piro and Ferdinando Di Cunto. Computational approaches to disease-gene prediction: rationale, classification and successes. FEBS Journal, 279(5):678-696, 2012.

[69] Clara Pizzuti. A multiobjective genetic algorithm to find communities in complex networks. Evolutionary Computation, IEEE Transactions on, 16(3):418-430, 2012.

[70] TS Keshava Prasad, Renu Goel, Kumaran Kandasamy, Shivakumar Keerthikumar, Sameer Kumar, Suresh Mathivanan, Deepthi Telikicherla, Rajesh Raju, 
Beema Shafreen, Abhilash Venugopal, et al. Human protein reference database2009 update. Nucleic acids research, 37(suppl 1):D767-D772, 2009.

[71] Matteo Re and Giorgio Valentini. Genes prioritization with respect to cancer gene modules using functional interaction network data. 2011.

[72] Alexander W Rives and Timothy Galitski. Modular organization of cellular networks. Proceedings of the National Academy of Sciences, 100(3):1128-1133, 2003.

[73] Francisco A Rodrigues, Guilherme Ferraz de Arruda, and Luciano da Fontoura Costa. A complex networks approach for data clustering. arXiv preprint $\operatorname{arXiv:1101.5141,2011.}$

[74] Marilyn Safran, Irina Dalah, Justin Alexander, Naomi Rosen, Tsippi Iny Stein, Michael Shmoish, Noam Nativ, Iris Bahir, Tirza Doniger, Hagit Krug, et al. Genecards version 3: the human gene integrator. Database, 2010:baq020, 2010.

[75] Mark Schena, Dari Shalon, Ronald W Davis, and Patrick O Brown. Quantitative monitoring of gene expression patterns with a complementary dna microarray. Science, 270(5235):467-470, 1995.

[76] Patrick S Tarpey, Raffaella Smith, Erin Pleasance, Annabel Whibley, Sarah Edkins, Claire Hardy, Sarah O'Meara, Calli Latimer, et al. A systematic, large-scale resequencing screen of $\mathrm{x}$-chromosome coding exons in mental retardation. Nature genetics, 41(5):535-543, 2009.

[77] Nicki Tiffin, Miguel A Andrade-Navarro, and Carolina Perez-Iratxeta. Linking genes to diseases: its all in the data. Genome Med, 1(8):77, 2009.

[78] MA Van Driel, K Cuelenaere, PPCW Kemmeren, Jack AM Leunissen, Han G Brunner, and Gert Vriend. Geneseeker: extraction and integration of human disease-related information from web-based genetic databases. Nucleic acids research, 33(suppl 2):W758-W761, 2005.

[79] Marc A van Driel, Jorn Bruggeman, Gert Vriend, Han G Brunner, and Jack AM Leunissen. A text-mining analysis of the human phenome. European journal of human genetics, 14(5):535-542, 2006.

[80] Oron Vanunu, Oded Magger, Eytan Ruppin, Tomer Shlomi, and Roded Sharan. Associating genes and protein complexes with disease via network propagation. PLoS computational biology, 6(1):e1000641, 2010. 
[81] Mahadevan Vasudevan and Narsingh Deo. Efficient community identification in complex networks. Social Network Analysis and Mining, 2(4):345-359, 2012.

[82] Dennis P Wall, Rimma Pivovarov, Mark Tong, Jae-Yoon Jung, Vincent A Fusaro, Todd F DeLuca, and Peter J Tonellato. Genotator: a disease-agnostic tool for genetic annotation of disease. BMC medical genomics, 3(1):50, 2010.

[83] Kenneth A Wallston. Hocus-pocus, the focus isn't strictly on locus: Rotter's social learning theory modified for health. Cognitive Therapy and Research, 16(2):183-199, 1992.

[84] David Warde-Farley, Sylva L Donaldson, Ovi Comes, Khalid Zuberi, Rashad Badrawi, Pauline Chao, Max Franz, Chris Grouios, Farzana Kazi, Christian Tannus Lopes, et al. The genemania prediction server: biological network integration for gene prioritization and predicting gene function. Nucleic acids research, 38(suppl 2):W214-W220, 2010.

[85] English Wikipedia. Partial map of the internet based on the january 15, 2005 data found on opte.org. http://commons.wikimedia.org/wiki/File: Internet_map_1024.jpg\#mediaviewer/File:Internet_map_1024.jpg, 2006. [Online; accessed 30-Sep-2014].

[86] Xuebing Wu, Rui Jiang, Michael Q Zhang, and Shao Li. Network-based global inference of human disease genes. Molecular systems biology, 4(1), 2008.

[87] Jianzhen Xu and Yongjin Li. Discovering disease-genes by topological features in human protein-protein interaction network. Bioinformatics, 22(22):2800-2805, 2006.

[88] Wei Yu, Marta Gwinn, Melinda Clyne, Ajay Yesupriya, and Muin J Khoury. A navigator for human genome epidemiology. Nature genetics, 40(2):124-125, 2008. 


\section{Appendix A}

\section{Sample output of genetic algorithm}

Here, we provide sample output generated by our GA-based method. This shows an excerpt containing out of generations for one of the 20 experiments we performed for Breast Cancer.

\section{A.1 Generations 1 to 20}

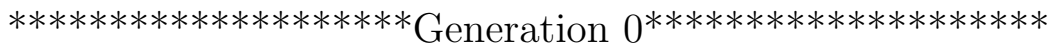

Generation's average fitness: 0.12110106472383664

Generation's best fitness: 0.18787878787878787

Generation's best individual:

$[7,3,2,6,1,7,5,7,3,3,5,1,1,3,1,6,1,3,2,3,5,1,1,5,6,5,2,0,1,2,0,3$, $7,0,6,7,5,2,6,3,5,3,6,1,3,3,1,6,0,0,7,5,5,0,4,2,5,5,1,1,2,6,6,3,4$, $2,1,5,6,5,5,5,5,0,7,2,6,5,7,4,3,3,7,5,7,4,5,1,1,1,4,3,2,1,2,3,4,4$, $2,5,2,2,3,4,1,3,5,2,0,0,6,0,1,1,2,7,7,7,5,4,6,7,5,7,3,2,1,7,5,7,5$, $0,4,1,5,5,0,5,2,3,0,5,5,2,1,6,4,7,5,1,7,6,2,4,2,7,1,1,0,1,1,5,6,7$, $5,1,2,6,3,1,7,2,0,6,1,0,5,2,1,2,5,4,0,0,1,0,4,7,6,1,1,6,4,5,6,1,5$, $6,6,4,4,1,7,5,7,0,4,6,5,1,1,1,4,5,3,4,0,7,4,6,6,5,1,1,1,7,2,7,7,7$, $3,6,3,1,0,0,5,1,0,0,6,5,1,6,2,2,0,5,5,2,0,5,7,3,1,4,1,0,3,7,7,0,6$, $6,2,7,5,7,2,6,5,2,6,0,4,1,1,7,5,7,2,6,6,2,5,4,4,5,7,5,0,2,4,1,1,1$, $1,3,5,1,5,4,7,7,4,6,4,3,5,1,3,1,4,7,7,4,4,5,2,7,3,2,2,3,1,6,0,1,4$, $7,7,2,1,3,7,4,0,1,2,7,0,6,0,0,2,3,2,6,2,4,0,7,4,7,6,5,7,6,4,0,1,3$, $0,3,6,2,5,4,1,4,7,1,1,1,6,5,3,4,1,4,6,2,6,3,7,4,1,3,4,4,4,5,5,3,3$, $7,2,5,6,0,7,0,0,1,1,2,7,3,5,7,7,3,4,4,5,1,6,2,0,5,5,1,3,3,7,7,3,3$, $3,2,6,6,0,7,7,6,4,4,1,3,1,6,1,1,1,5,1,6,3,2,4,1,4,6,7,4,0,3,7,7,6$, $2,7,1,5,4,5,0,2,4,2,1,2,1,5,1,1,0,4,7,0,1,4,2,7,6,4,4,4,1,5,5,3,4$, 
$3,5,2,1,5,4,0]$

Generation's best community (list of genes):

ATAD2 NBN RAD52 LRIT3 H2AFX FAS AP2M1 TDP1 ATF1 BAP1 VCP RAD51D DHCR24 THRB MDC1 RIPK3 DBNL CREBBP ATR HIPK3 PER3 GPR25 TP53BP1 RASD2 ZHX1 MDM2 MDM4 TAB3 RAD54B NFYA JUND HIST2H2AB PTK2B ESR1 DDX5 CDC25A TFCP2 SUMO2 CDS1 BCCIP NR2C2 ZNF280B HSPA5 DCLRE1A CASP7 XRCC3 ZCCHC8 JUNB UBE2U IKBKB DCLRE1C NR5A2 SMARCA4 RCOR1 CTBP1 SMAD3 IKBKG GTF2B APOL2 CNTN2 TAOK3 SLC9A3 ATG12 HDAC2 RALBP1 CBR3 PPARA VRK2 HDAC1 ULK1 DEDD CLSPN AKT1 AURKB TACC1 DAXX MRAS PBK RBBP4 ERCC6 TP63 C1orf131 KPNA2 TP53INP1 VRK1 YWHAH EME1

Number of genes inside the community: 87

$* * * * * * * * * * * * * * * * * * * *$ Generation $1 * * * * * * * * * * * * * * * * * * * * *$

Generation's average fitness: 0.11793895337436579

Generation's best fitness: 0.16175507531106745

Generation's best individual:

$[0,7,6,2,1,1,6,7,5,5,5,6,2,0,1,0,1,7,4,3,1,7,1,4,7,0,2,3,2,6,4,1$, $4,1,4,6,6,7,3,5,6,6,7,0,5,5,1,3,7,7,0,3,2,7,4,1,3,3,7,3,6,3,3,1,2$, $3,4,1,1,7,0,2,2,3,4,5,1,5,2,3,4,4,2,3,3,7,5,0,1,4,5,4,6,1,0,0,1,7$, $6,2,7,6,4,1,4,5,3,6,3,6,1,0,3,7,0,4,2,7,4,7,0,7,5,3,7,7,0,2,0,5,3$, $6,5,3,3,3,0,4,4,7,2,1,5,7,1,4,2,5,0,2,3,5,3,2,3,0,4,6,2,7,0,2,6,7$, $3,4,7,5,1,4,0,5,4,0,3,7,2,5,7,0,4,0,3,3,2,6,1,3,0,1,3,1,4,2,7,4,4$, $4,0,1,0,0,1,5,5,1,4,2,2,1,1,1,7,3,0,0,7,2,3,7,4,4,2,1,2,1,5,2,7,2$, $2,6,3,7,2,4,1,0,2,3,3,2,6,5,2,7,0,0,6,6,2,2,5,3,5,6,4,4,0,2,1,0,0$, $3,4,1,0,1,5,0,1,4,5,4,4,6,2,0,1,2,5,3,1,7,5,4,1,4,6,3,1,2,3,0,0,6$, $5,5,2,1,5,1,7,5,1,4,0,3,0,7,0,3,6,4,7,3,5,1,7,2,5,2,1,2,2,1,5,3,1$, $4,5,1,1,3,5,0,5,5,3,1,0,3,2,7,0,5,3,2,6,4,6,7,4,2,3,6,5,0,0,4,0,2$, $1,0,0,4,6,0,3,0,2,0,4,2,0,0,1,4,7,0,1,5,1,2,2,4,4,0,1,1,6,1,5,5,3$, $5,1,6,1,7,6,6,5,1,2,5,2,4,4,6,6,5,0,7,4,0,3,5,0,1,7,3,2,6,3,4,4,4$, $4,5,1,2,7,7,1,0,2,0,6,7,2,2,3,2,4,7,3,5,2,1,3,3,0,2,0,0,7,0,0,4,5$, $5,2,0,6,2,5,1,4,2,5,3,4,0,5,0,1,2,7,6,1,6,4,6,1,5,5,5,4,7,4,1,1,3$, $7,2,1,5,4,3,4]$

Generation's best community (list of genes):

ATAD2 MAST2 LRIT3 H2AFX RAD9A AP2M1 NCOA4 WRN BAP1 SPDEF RAD51AP1 RRM2B RAD50 SUMO1 MDC1 DBNL HSPA1A MAGEB18 RRM1 UBE2I TP53BP1 MAGI3 IRS4 ESR1 GSK3B TSSK1B NOL3 FANCE SUMO2 CDS1 
BCCIP ZNF280B SP1 COX5B MSH3 CUL9 PTK2 RB1 DENND5A NCL HIST3H3 TIPARP CTBP1 RNF14 PPM1B CASP6 PPP2R2B JUN GPS2 MAP2K6 CNTN2 FANCI DNAJA3 FASLG DDX17 AIMP2 MARK1 FANCF EEF2 FOXA1 KRAS HDAC1 PATZ1 NR4A1 NOD1 NRIP1 TRAF2 TP53INP1 PGR SAFB FBXO11 TOP2A DRD2

Number of genes inside the community: 73

*********************** Generation $2 * * * * * * * * * * * * * * * * * * * *$

Generation's average fitness: 0.12043707089977106

Generation's best fitness: 0.15571428571428572

Generation's best individual:

$[0,7,4,6,5,6,4,7,1,3,3,3,0,3,2,1,6,2,7,0,1,3,3,6,7,1,6,3,1,7,5,1$, $6,4,7,2,0,0,4,6,6,6,5,0,5,7,2,4,2,7,2,2,4,7,5,1,0,0,5,3,5,3,6,7,0$, $3,4,2,5,4,4,7,1,3,1,1,7,2,3,1,7,3,0,7,3,2,0,0,2,7,7,2,3,2,4,3,5,1$, $2,6,1,1,1,2,3,5,3,3,1,0,6,7,0,2,1,0,6,2,4,4,1,7,6,0,6,4,3,5,1,6,2$, $2,5,1,0,0,1,7,5,2,0,5,5,2,5,7,7,1,0,0,0,1,2,2,6,3,5,0,0,3,0,4,4,6$, $2,6,6,2,3,3,1,2,6,6,7,3,1,1,4,2,7,3,2,4,4,0,5,5,2,4,7,7,1,5,2,1,1$, $4,0,7,3,7,3,4,2,6,3,1,0,3,2,5,1,0,5,3,3,6,7,0,4,1,1,0,5,5,5,6,1,0$, $7,1,6,7,1,2,2,1,2,3,1,2,5,0,1,5,3,3,5,6,3,3,3,6,7,0,3,1,3,4,7,0,3$, $0,2,4,3,4,7,7,0,7,3,6,5,0,0,2,5,7,1,1,1,2,6,1,3,2,0,4,5,0,1,5,6,3$, $2,6,4,4,5,6,2,6,2,5,3,4,4,7,6,5,7,3,6,1,2,7,4,4,6,7,2,7,3,1,2,7,5$, $2,0,3,2,2,2,0,1,5,1,5,6,0,1,0,0,4,5,5,2,4,7,6,0,1,7,4,2,3,2,2,3,5$, $7,7,0,7,0,3,0,2,6,5,7,2,0,7,3,5,3,6,2,3,6,5,4,4,3,6,4,3,7,2,7,4,1$, $6,1,3,5,7,2,7,7,5,6,0,0,0,0,3,3,0,3,6,7,7,2,3,4,3,4,2,6,5,7,5,0,3$, $4,6,5,1,4,1,4,6,2,3,6,7,5,3,4,4,0,1,0,6,5,3,7,5,1,6,7,2,7,2,3,5,4$, $4,6,1,4,3,7,5,6,2,1,3,0,7,0,7,0,3,4,1,1,4,4,6,2,4,2,1,1,3,2,7,3,6$, $6,0,3,5,4,6,2]$

Generation's best community (list of genes):

FRK APPL1 RAD9A TAB2 TDP1 NCOA4 SPDEF KAT5 CSTF1 ESRRB ING5 KAT2B BACH1 E2F1 CUL3 PPP1R13L VDR ABL1 NUFIP1 GPR25 NCOA1 HSBP1 PLK3 FEZ1 MRE11A MSH6 RELA CDC25A PTN GSN HSP90AA1 BAG1 NR2C2 BCL2 RAD51B IL17RD CASP7 PRMT1 ING4 MAST1 RCHY1 POU2F1 NCL MAP4K4 PRKDC NR1H4 JUN NKX2-1 NR3C1 RECQL5 SMAD6 MRPS34 FOXA1 ETS2 HTT DARS BID ERCC6 PPP2R5D NR2C1 PGR MED17 SHISA5

Number of genes inside the community: 63

$* * * * * * * * * * * * * * * * * * * *$ Generation $3 * * * * * * * * * * * * * * * * * * * *$

Generation's average fitness: 0.12294180279277041 
Generation's best fitness: 0.15876288659793814

Generation's best individual: $[1,5,5,0,7,6,2,6,3,3,1,1,0,6,2,3,1,2,3,6$, $6,1,2,5,0,7,6,3,6,5,1,5,5,3,4,0,5,4,2,7,4,1,3,0,0,3,1,4,0,5,2,0,6$, $5,2,7,2,3,2,7,6,4,7,1,6,3,0,4,0,3,6,7,4,2,4,0,0,3,0,2,4,7,1,5,5,5$, $3,6,7,0,0,1,5,4,5,4,1,6,4,0,5,1,1,6,0,4,4,2,6,1,3,0,1,4,6,4,0,5,6$, $5,6,3,5,4,7,7,0,5,3,6,7,1,0,0,1,2,6,0,2,2,2,1,2,5,0,3,7,7,1,1,5,0$, $5,2,2,1,2,2,4,1,5,2,2,1,3,3,6,6,0,2,1,0,5,7,6,7,6,1,5,3,1,1,2,2,7$, $4,2,5,0,6,0,7,5,2,6,3,7,0,5,4,0,4,0,6,1,7,4,3,5,0,6,4,1,7,6,4,0,2$, $1,5,6,0,0,7,6,0,1,7,1,5,1,5,3,1,0,7,2,2,4,2,6,7,4,0,5,5,3,0,3,5,2$, $3,0,6,3,0,6,4,7,7,3,4,3,6,6,5,0,2,7,0,0,1,6,4,1,7,1,0,2,0,0,2,2,3$, $5,0,5,7,4,5,2,7,5,0,5,7,2,7,1,5,5,3,6,3,7,3,6,3,7,1,5,0,3,0,1,5,6$, $0,7,5,6,6,5,1,6,3,0,7,1,2,6,2,5,2,0,4,6,0,4,2,7,5,1,3,7,7,6,6,3,6$, $5,7,6,7,6,0,1,2,3,3,2,3,5,6,4,3,4,3,0,0,5,2,0,1,0,0,7,4,3,5,7,2,3$, $4,7,6,7,5,5,7,5,0,5,0,1,0,0,1,7,5,7,0,6,5,1,6,4,4,7,6,4,4,1,4,7,4$, $4,5,6,4,2,1,6,5,1,2,3,3,4,5,7,5,2,3,7,0,5,1,1,1,0,3,2,6,6,2,2,6,6$, $3,5,5,3,4,0,5,6,4,3,3,1,0,4,1,4,6,5,1,1,4,6,7,6,0,5,5,1,6,7,3,6,5$, $4,6,2,2,7,1,3,5,2,5,0,2,7,0,6,3,3,5,7]$

Generation's best community (list of genes):

6885 PALB2 NBN H2AFX FAS FANCD2 AP1B1 BAP1 RAD51AP1 MRC1 HRAS HSPA1A E2F1 CUL3 MVP ATR RBBP8 PIAS1 UBE2I RAD54L RASD2 TERF2 MDM4 RNF4 FEZ1 MSH6 UIMC1 MOB4 EP300 HSP90AA1 CHUK SAFB2 BCL2 WNT2B DCLRE1A ESRRA CCND1 IKBKB AIF1 SMAD3 CDC14B CHEK1 GPS2 RECQL5 PAK6 CBR3 MRPS34 AURKA ULK1 SLC9A3R2 CLSPN EVL RHEBL1 AKT1 SETD7 ETS1 ERCC6 TRAF2 TIRAP TP53INP1 MED17

Number of genes inside the community: 61

*********************** Generation $4 * * * * * * * * * * * * * * * * * * * * *$

Generation's average fitness: 0.125389003768463

Generation's best fitness: 0.15843023255813954

Generation's best individual:

$[0,4,2,2,1,0,6,2,5,0,0,6,5,0,6,0,0,2,4,5,2,4,2,2,5,0,5,4,2,0,5,5$, $6,2,2,0,7,4,2,3,1,7,1,3,5,4,1,6,5,3,7,2,5,7,0,6,7,6,1,2,2,4,1,2,3$, $3,3,0,5,7,0,3,1,1,3,6,1,6,3,2,5,6,2,6,6,6,5,1,7,2,6,3,6,4,3,1,2,5$, $7,0,4,6,3,4,0,7,4,0,0,2,5,2,3,5,0,7,3,4,6,1,1,6,7,3,1,1,3,6,0,0,1$, $1,4,5,1,3,1,5,0,1,5,1,2,5,7,0,1,4,6,3,1,7,6,7,4,7,2,1,7,1,2,3,1,6$, $2,7,3,0,3,3,1,1,1,7,0,1,0,5,1,4,2,1,0,7,0,5,6,0,6,0,6,0,7,3,6,0,3$, $4,1,2,3,0,7,1,3,7,3,7,0,4,7,0,4,4,3,6,5,7,6,1,4,2,4,3,6,5,6,5,6,0$, 
$4,6,6,4,4,3,3,0,2,1,1,7,6,3,6,6,4,5,7,3,3,5,1,4,7,2,6,5,5,2,4,4,0$, $7,5,0,5,2,1,7,1,3,5,5,6,4,7,4,1,7,0,5,0,2,7,4,7,3,1,7,7,3,5,0,3,1$, $6,2,2,6,1,5,4,3,6,4,7,3,6,6,6,3,5,0,6,2,7,4,4,5,6,5,2,4,0,6,3,6,5$, $7,6,5,5,6,4,6,2,6,1,6,7,4,6,5,2,3,6,5,7,6,0,2,1,3,5,4,4,5,0,7,2,4$, $1,7,5,1,6,4,5,0,5,3,7,7,5,3,1,4,4,5,7,6,5,2,3,5,7,3,1,3,7,3,2,6,7$, $3,4,3,7,2,4,5,3,3,0,4,7,3,3,3,5,7,6,1,3,4,4,5,1,6,1,3,0,0,0,1,3,4$, $2,6,4,2,5,0,6,1,7,0,2,2,3,4,4,3,1,6,7,0,2,1,5,5,3,1,5,5,2,7,7,1,3$, $7,2,2,0,1,0,2,7,7,2,6,3,5,3,5,4,0,2,4,7,3,3,0,5,4,3,2,2,4,3,5,3,7$, $4,2,6,6,3,4,1]$

Generation's best community (list of genes):

ATAD2 RECQL4 STRADB BAP1 VCP SNRK KAT5 ATG13 SUMO1 THRB TFAP2C ING1 ABL1 RPA1 FOXO3 TMF1 RBBP8 PIAS1 NCOA1 MED1 UBE2I GCN1L1 ETV5 MDM2 MDM4 CDHR2 FEZ1 C11orf30 PSMB9 TOPBP1 HIST2H2AB MOB4 FANCA GPRASP2 TNFSF10 TIMM13 PRMT1 DNAJC7 TOP3A RB1 DENND5A BRE SMARCA4 APEX1 NR3C1 BCL3 DNAJA3 TINF2 FASLG MARK1 SERPINH1 RARA TNFRSF1A REV3L PPARG MRAS NRIP1 STAT5A PPP2R5C PEA15 PDGFRB

Number of genes inside the community: 61

$* * * * * * * * * * * * * * * * * * * *$ Generation $5 * * * * * * * * * * * * * * * * * * * *$

Generation's average fitness: 0.1278126863951778

Generation's best fitness: 0.17342657342657342

Generation's best individual:

$[3,3,3,6,6,1,6,4,0,5,2,5,1,5,2,4,7,3,3,0,1,7,7,4,0,6,2,1,6,1,7,6$, $7,5,6,6,6,5,4,2,0,4,6,1,0,2,2,7,0,3,4,3,6,7,2,7,6,5,7,2,2,1,6,0,5$, $4,3,5,6,5,3,7,2,1,3,2,1,3,2,3,0,1,1,2,7,5,6,5,5,7,2,4,6,7,2,4,4,6$, $6,3,1,1,6,0,0,6,3,6,0,5,4,3,1,1,6,2,7,2,2,7,0,0,4,5,1,5,7,4,5,0,2$, $0,4,0,1,5,0,4,5,5,7,1,3,5,3,2,4,0,6,5,5,6,1,1,1,2,4,4,0,3,7,2,0,4$, $3,2,7,4,7,0,4,3,2,5,7,3,0,5,7,1,3,4,2,4,3,4,4,4,4,3,7,7,6,3,6,7,1$, $0,3,7,5,2,7,5,0,0,0,4,3,1,2,5,6,4,0,3,5,3,6,2,4,7,3,3,6,1,3,1,0,0$, $4,4,5,7,7,7,5,3,3,4,3,3,7,1,4,7,4,7,7,3,6,4,7,4,0,4,1,2,1,3,7,0,2$, $7,1,1,5,1,2,1,0,4,7,4,0,0,6,5,4,3,3,2,3,1,5,3,0,2,4,5,2,4,6,2,7,4$, $6,3,7,0,0,4,6,4,6,4,1,5,1,1,2,1,7,7,7,7,6,0,5,0,2,0,3,4,7,4,6,0,5$, $6,2,0,5,0,3,5,0,7,4,6,2,2,0,0,4,7,3,3,0,0,3,0,0,6,5,0,0,5,6,6,0,7$, $0,1,2,0,5,5,1,3,1,1,5,0,0,2,2,4,2,7,6,1,1,6,1,3,0,5,4,6,5,2,0,0,3$, $6,2,0,4,6,6,7,2,1,6,6,6,6,2,2,3,3,1,2,3,1,1,2,1,4,7,1,5,2,3,3,4,1$, $0,3,3,3,7,6,4,2,4,2,0,7,2,4,4,1,7,1,5,5,1,4,3,6,4,3,0,7,1,0,7,5,4$, 
$3,3,2,2,2,4,3,7,4,7,5,0,3,3,3,2,6,1,5,2,3,6,1,2,4,5,5,3,2,4,3,4,0$, $1,0,5,0,2,5,1]$

Generation's best community (list of genes):

MAST2 RAD52 RAD9A CDK19 FKBP5 ATF1 TOPORS ATG13 SUMO1 RPA2 MRC1 BACH1 E2F1 ATR HIPK3 RPA1 PIAS1 UBE2I FAM175A CASP1 ATN1 RPL5 PTN SUMO2 SP1 YLPM1 HMG20B JUNB RASGRP3 ACACA CUL9 PTK2 PIAS4 KIAA1377 CDK7 CCNH SMAD3 IKBKG UBE2D3 ATG12 ERCC2 HDAC2 GTF2F1 AIMP2 RPA3 HDAC1 SLC9A3R2 DEDD E2F4 RARA CLSPN RBMX DAXX DARS RAD54L2 ELK4 IARS HIF1A FAIM2 PDGFRB

Number of genes inside the community: 60

$* * * * * * * * * * * * * * * * * * * *$ Generation $6 * * * * * * * * * * * * * * * * * * * *$

Generation's average fitness: 0.1301322682531589

Generation's best fitness: 0.16981132075471697

Generation's best individual:

$[0,6,6,4,1,1,6,6,2,7,1,4,5,6,4,5,7,3,6,0,3,5,4,2,6,3,7,0,7,3,1,4$, $7,7,1,2,1,7,1,3,6,5,6,4,5,5,4,3,7,5,3,6,0,3,3,0,7,5,0,7,4,5,1,3,7$, $6,3,0,4,3,4,4,2,2,1,5,1,0,0,1,4,4,0,3,5,2,0,2,1,6,2,6,1,7,0,0,5,7$, $5,7,3,1,5,3,0,2,4,5,6,6,3,0,7,0,7,0,4,4,0,1,1,4,5,2,3,4,6,7,1,2,6$, $1,5,4,4,6,4,2,1,7,5,4,1,0,1,5,1,4,5,7,0,7,2,5,4,1,4,5,2,1,2,5,6,1$, $6,7,3,1,1,4,5,5,7,0,4,3,3,4,6,7,0,4,3,6,6,0,7,0,0,1,3,0,3,0,7,3,6$, $0,5,0,4,3,3,5,1,3,0,4,4,2,3,7,3,1,1,3,2,2,7,2,0,1,2,7,2,1,3,2,5,3$, $6,0,4,0,1,7,4,2,4,7,2,3,4,0,3,4,3,1,6,7,0,5,2,6,3,3,2,4,6,5,7,3,3$, 4, 0, 2, 1, 7, 7, 1, 2, 5, 6, 6, 4, 6, 4, 4, 7, 3, 3, 4, 1, 1, 4, 6, 4, 1, 1, 5, 6, 5, 7, 0, 7, 7, $3,0,0,1,4,1,3,0,2,0,1,0,3,6,1,0,1,3,0,3,5,3,0,0,0,6,5,1,2,3,3,5,2$, $4,4,1,0,2,4,7,6,2,1,4,3,6,2,5,5,5,6,4,5,6,1,1,4,1,6,4,2,0,1,1,7,7$, $4,7,7,0,3,5,4,3,2,6,4,7,2,6,2,1,0,5,2,3,7,7,7,0,0,7,3,5,2,5,5,1,2$, $5,2,3,2,5,6,6,1,5,4,4,1,4,1,4,3,1,4,1,4,5,4,7,1,7,5,3,7,6,5,6,5,2$, $1,2,6,1,1,4,6,0,0,0,2,2,5,0,0,1,5,2,5,1,1,5,5,5,3,7,2,7,5,5,3,0,7$, $5,1,1,5,3,1,2,7,4,0,3,4,6,4,7,6,2,6,0,5,3,5,0,7,0,3,2,4,4,6,6,4,4$, $4,7,6,0,4,2,7]$

Generation's best community (list of genes):

ATAD2 MAST2 PALB2 FANCD2 ERCC6L TAB1 TRAF6 SNRK CSTF1 SUMO1 ING5 MDC1 IKBKAP E2F1 ING1 ABL1 NUFIP1 RBBP8 RHEB MSH2 TP53BP1 GCN1L1 TERF2 MDM4 RNF4 MUS81 MAGI3 ESR1 EP300 CASP3 RYBP BAG1 SP1 IL17RD MED12L SETDB1 PIAS4 NCL KIAA1377 MDN1 BRE CTBP1 RNF14 CDK7 NRG1 PEBP1 CHEK1 MAP2K6 NR3C1 PA2G4 ZMIZ1 SMAD6 MAP4K1 
HMGB1 MAP1LC3C E4F1 DGKZ ATRX NR0B2 SRY SERPINH1 RARA UBA1 ETS2 NAIP DAXX HIPK2 RAD54L2 TERF2IP ERCC6 RAD17

Number of genes inside the community: 71

$* * * * * * * * * * * * * * * * * * * *$ Generation $7 * * * * * * * * * * * * * * * * * * * *$

Generation's average fitness: 0.1323757861344664

Generation's best fitness: 0.1766323024054983

Generation's best individual:

$[0,7,6,7,4,4,6,6,6,2,4,2,2,4,0,0,1,5,3,0,5,0,0,3,7,4,3,3,0,0,1,5$, $4,5,4,3,0,5,5,3,1,4,7,4,6,0,5,3,4,6,5,1,6,0,1,3,4,1,3,7,3,5,2,6,4$, $0,0,7,1,0,0,4,0,7,3,7,0,4,5,1,6,2,0,6,3,4,0,2,2,1,7,1,0,0,3,1,1,1$, $5,0,3,1,4,4,1,4,6,4,2,3,2,7,4,3,6,2,3,7,6,7,1,5,6,4,0,3,2,7,0,6,1$, $1,4,6,5,6,0,7,2,7,2,4,6,2,1,1,6,3,2,2,6,0,5,3,7,0,6,0,4,6,3,0,0,4$, $0,3,0,2,6,3,4,4,7,0,1,1,1,7,4,2,6,5,7,7,2,0,7,5,6,7,4,1,2,3,5,3,1$, $7,6,5,6,2,7,1,7,6,6,5,3,1,1,6,0,0,7,7,0,2,2,2,7,0,4,6,2,2,7,5,5,3$, $4,6,7,4,7,2,1,4,6,2,5,0,2,6,3,0,0,3,3,7,4,4,7,4,6,4,7,2,6,6,5,3,1$, $4,2,7,5,2,3,2,1,2,5,4,5,0,4,6,7,3,2,3,2,0,7,2,1,3,1,1,7,2,1,7,1,1$, $7,6,1,0,7,0,5,5,4,1,1,4,3,6,5,1,5,4,0,5,2,2,4,0,7,4,0,1,5,7,4,5,1$, $5,6,3,7,2,7,3,7,5,3,3,4,1,0,0,0,1,2,3,0,4,7,6,3,2,2,1,5,5,7,1,4,5$, $7,7,0,1,6,5,6,3,3,3,0,3,1,7,2,6,0,6,1,2,2,5,4,0,3,2,7,0,4,4,5,2,2$, $1,0,0,6,1,3,4,5,5,7,3,3,4,4,6,4,1,6,0,4,3,7,6,4,2,6,3,4,6,6,3,7,6$, $1,3,6,4,1,3,2,6,6,2,4,0,0,5,2,3,3,0,5,5,2,0,6,5,2,0,4,7,6,3,1,3,0$, $4,5,6,0,0,3,7,7,4,5,5,3,7,4,1,5,0,3,4,7,0,5,7,0,2,7,1,5,3,1,7,1,2$, $6,0,0,5,1,6,7]$

Generation's best community (list of genes):

H2AFX FANCD2 RECQL4 BLM DMC1 TGFB1I1 RAD50 ING5 RIPK3 HRAS TFAP2C HSPA1A KAT2B E2F1 CREBBP ABL1 TMF1 RBBP8 TP53BP1 BRAP JUND TOPBP1 MRE11A GSK3B PTN GPRASP2 SUMO2 CDS1 COX5B XRCC6 RB1 HIST3H3 BRE NR1H3 PRKDC NR5A2 SMARCA4 AIF1 PTS CDK7 IKBKG CHEK1 GPS2 TXN C16orf53 PMEPA1 HMGB1 TINF2 CABLES1 DDX17 BFAR UBE2L3 SRY UBA1 NAIP UPF1 KPNA2 MED17 RBAK TOP2A CDK1

Number of genes inside the community: 61

************************Generation $8 * * * * * * * * * * * * * * * * * * * *$

Generation's average fitness: 0.13455985118457658

Generation's best fitness: 0.17372881355932204

Generation's best individual:

$[0,0,6,2,7,5,2,3,3,4,7,1,3,3,4,0,1,7,1,4,6,4,1,5,6,7,4,1,2,7,0,6$, 
$7,5,6,6,6,5,4,2,0,4,6,1,0,2,2,7,0,3,4,3,6,7,2,7,6,5,7,2,2,1,6,0,5$, $4,3,5,6,5,3,7,2,1,3,2,1,3,2,3,0,1,1,2,7,5,6,5,5,7,2,4,6,7,2,4,4,6$, $6,3,1,1,6,0,0,6,3,6,0,5,4,3,1,1,6,2,2,5,3,5,1,7,4,5,1,5,1,4,6,6,4$, $6,1,3,4,6,2,6,7,1,5,7,1,4,6,2,0,5,4,4,7,0,4,4,6,5,7,5,4,1,4,3,0,6$, $2,1,6,4,7,6,4,0,7,6,0,5,0,4,3,6,6,2,0,0,5,5,3,4,0,7,4,7,4,2,4,6,5$, $1,7,1,4,0,1,2,2,4,2,7,5,4,5,5,1,3,0,5,2,6,3,7,6,4,7,2,1,1,1,7,6,2$, $5,6,7,7,5,5,1,4,1,7,7,4,7,2,2,5,3,4,0,3,5,4,5,4,2,7,6,5,6,3,4,7,1$, $3,2,0,7,7,0,5,3,4,4,0,7,4,5,1,4,6,6,1,6,4,5,7,2,7,0,5,6,3,1,6,7,6$, $4,2,2,5,6,5,4,7,3,7,1,1,1,2,3,1,0,1,6,7,5,3,2,6,4,5,5,1,4,2,0,2,6$, $2,5,0,5,3,3,4,3,2,6,3,1,1,0,7,5,4,0,1,5,5,4,2,6,6,1,7,2,6,0,1,1,4$, $4,6,3,3,7,0,0,2,1,2,0,7,6,5,0,6,5,2,5,6,3,4,6,2,3,3,4,4,0,3,5,5,5$, $1,5,2,1,1,0,1,7,0,7,0,3,5,7,4,6,5,4,2,1,4,3,3,2,4,7,2,4,7,6,2,7,0$, $3,1,1,0,2,4,2,5,7,6,4,3,3,7,0,2,5,6,1,3,7,4,2,1,5,2,3,3,3,5,3,5,4$, $2,4,7,6,0,3,0,3,4,2,6,3,3,3,5,2,3,2,7,6,3,6,6,1,1,7,6,2,6,5,2,6,1$, $3,5,2,6,3,5,5]$

Generation's best community (list of genes):

NBN H2AFX LGTN AP2M1 CDK19 ATF1 TOPORS ATG13 SUMO1 RPA2 MRC1 BACH1 E2F1 ATR HIPK3 ABL1 RPA1 PER3 PPM1L MED1 MSH2 MDM4 RAD54B TRADD TSSK1B NOL3 HSP90AA1 HSPA5 SP1 SAFB2 COX5B CHD3 XRCC6 PLK1 POU2F1 PRKDC CDK7 CASP14 CCNH IKBKG ERCC3 CHEK1 RFWD2 TXN BUB1B XIAP HMGB1 SLC9A3 ERCC2 BFAR KRAS UBE2L3 MARK2 CSNK2A2 PPP2R2A NR4A1 PBK RBBP4 SAFB PARN NR2F1

Number of genes inside the community: 61

********************** Generation $9 * * * * * * * * * * * * * * * * * * * *$

Generation's average fitness: 0.1369551159777728

Generation's best fitness: 0.17246273953158267

Generation's best individual:

$[7,0,7,1,5,0,6,7,0,1,0,2,3,2,2,0,2,4,5,5,2,6,1,7,0,0,4,4,3,1,3,7$, $2,2,7,6,3,1,5,6,0,4,3,2,7,2,0,4,6,4,1,3,6,4,2,3,4,1,7,7,5,6,2,2,2$, $4,0,7,4,6,6,2,5,4,1,7,0,2,0,3,0,3,6,6,6,0,5,1,1,4,1,5,5,4,7,4,7,1$, $6,2,7,6,2,5,1,4,4,3,3,0,0,6,6,6,1,3,2,5,3,5,1,7,4,5,1,5,1,4,6,1,7$, $4,5,2,1,7,4,1,3,1,6,4,1,5,1,1,6,7,2,7,0,0,6,5,7,4,1,1,7,4,5,1,0,0$, $6,2,2,3,5,0,3,1,5,7,6,6,2,0,3,7,2,0,0,5,6,4,5,6,1,6,3,6,6,4,3,0,3$, $5,3,1,5,7,1,6,6,0,7,4,6,7,6,7,4,5,4,3,6,3,2,0,2,3,1,6,0,2,0,6,4,1$, $5,1,7,4,4,6,6,5,1,6,6,7,7,0,2,4,6,6,4,3,5,0,2,5,4,4,4,6,3,2,0,5,5$, $1,3,7,3,5,0,0,1,0,6,2,0,3,4,7,3,2,7,0,4,4,6,7,7,3,7,4,3,6,3,2,5,6$, 
$5,2,3,1,1,5,4,3,6,4,7,3,6,6,6,3,5,0,6,2,7,4,4,5,6,5,2,4,0,6,3,6,5$, $7,6,5,0,3,7,2,6,6,1,3,6,0,2,3,4,6,7,3,3,1,4,3,7,7,1,7,3,3,0,2,7,7$, $5,0,7,5,4,2,4,3,2,2,7,7,7,1,6,3,4,0,5,4,1,3,4,4,5,5,2,3,0,4,0,4,4$, $7,0,7,3,6,2,5,3,1,7,4,6,7,1,2,7,3,1,1,5,7,5,4,3,4,4,3,4,4,4,3,4,0$, $1,2,2,1,2,0,7,1,5,0,6,3,0,4,2,2,5,1,6,7,5,1,2,6,4,2,2,2,4,2,3,5,3$, $7,2,0,6,2,1,5,1,7,7,3,7,3,0,1,0,3,3,4,3,4,4,6,5,5,7,1,0,3,0,5,1,2$, $1,0,4,3,2,2,2]$

Generation's best community (list of genes):

PRKAA2 BRIP1 AP2M1 FKBP5 RXRB PLAGL1 TGFB1I1 CSTF1 THRB MDC1 BRD7 KAT2B CREBBP VDR ABL1 RPA1 PER3 CXXC5 PIAS1 IFI16 MED1 MSH2 TP53BP1 BRAP ZHX1 MDM2 RARG C11orf30 RASA4 TSSK1B NOL3 NR2C2 MCPH1 RAD51B CHD3 LRIF1 RB1 CTBP1 APEX1 NR3C1 UBE3A XIAP ARHGEF1 AIMP2 HDAC1 NR0B2 SLC9A3R2 SERPINH1 UBA1 ETS2 PPARG DARS NRIP1 RAD17 TIRAP KPNA2 MED17 TOP2A FAIM2

Number of genes inside the community: 59

$* * * * * * * * * * * * * * * * * * * *$ Generation $10 * * *$

Generation's average fitness: 0.13936486620546543

Generation's best fitness: 0.17532467532467533

Generation's best individual:

$[0,2,4,1,1,4,6,4,0,5,2,5,1,3,0,4,4,0,6,0,1,3,7,4,7,6,4,1,6,1,7,4$, $4,1,0,6,0,5,5,6,6,1,3,0,5,3,3,1,1,2,2,4,6,3,0,1,3,7,2,6,3,5,7,6,0$, $1,2,7,3,4,0,4,7,1,4,7,5,7,2,6,4,1,2,0,2,6,2,6,3,3,6,2,0,7,4,0,4,3$, $2,3,3,6,0,2,3,6,3,4,2,2,1,2,4,2,1,4,0,3,2,2,1,4,7,6,1,4,6,1,2,5,5$, $6,5,0,3,3,1,3,1,2,1,1,2,4,7,3,5,5,3,7,1,6,0,1,2,6,4,3,5,3,0,1,5,1$, $7,6,6,0,4,1,6,6,2,7,6,7,0,1,7,1,6,7,0,2,5,5,1,4,4,1,7,7,6,2,4,4,5$, $7,3,5,7,7,4,7,0,3,2,3,6,3,7,2,5,4,1,1,0,1,1,5,7,3,1,0,7,4,1,4,6,2$, $5,0,2,3,5,7,5,7,1,5,0,6,0,4,4,4,4,5,4,6,4,4,2,4,3,2,4,2,6,7,1,5,1$, $6,3,4,5,3,7,1,7,1,6,6,5,2,3,1,7,6,6,4,0,5,2,7,2,3,2,3,4,3,3,1,0,3$, $3,5,3,4,0,5,7,1,5,0,0,3,3,1,3,0,4,2,4,2,5,0,0,4,3,0,1,6,1,5,5,5,1$, $3,5,6,7,3,0,6,7,5,1,6,6,4,2,0,6,6,5,2,2,2,0,6,5,5,0,3,0,1,2,3,2,6$, $1,3,2,4,0,5,1,1,4,2,4,2,0,7,7,1,3,3,3,5,4,1,0,2,2,6,2,1,2,2,4,6,2$, $0,1,7,7,6,4,0,4,3,1,5,6,7,4,6,6,2,6,7,2,5,2,0,4,0,6,2,7,0,7,6,0,6$, $2,3,2,0,1,4,5,1,6,4,4,6,5,2,3,1,3,2,5,2,6,6,0,1,3,1,5,1,3,2,5,6,1$, $7,1,5,1,0,4,1,2,6,2,6,2,3,0,4,5,1,4,7,3,7,6,4,1,1,3,1,1,7,7,5,5,7$, $7,5,2,2,2,0,3]$

Generation's best community (list of genes): 
PRKAA2 ATAD2 RAD52 RAD9A CDK19 FKBP5 WRN AP1B1 ERBB3 OTUD7B SPDEF FADD ATG13 RPA2 RRM1 VDR ABL1 RPA1 RAD51AP2 NCOA1 RHEB NTHL1 UBE2I ETV5 CASP1 RARG RNF4 NFYA MSH6 RPL5 IRS4 ESR1 RYBP ULK2 IRS1 CHUK NR2C2 SAFB2 CHD3 MSH3 XRCC6 PIAS4 ESRRA PLK1 DCLRE1C CFLAR SMAD3 PPP2R2B GTF2B GPS2 NR3C1 SRA1 DNAJA3 ATG12 XRCC1 MAP1LC3C ZNF350 FANCF FOXA1 ULK1 NAIP PPARG DAXX RBBP4 STAT5A MAP3K5 ETS1 TERF2IP PTTG1 TRAF2 TICAM1 SAFB PARN MED17 SHISA5

Number of genes inside the community: 75

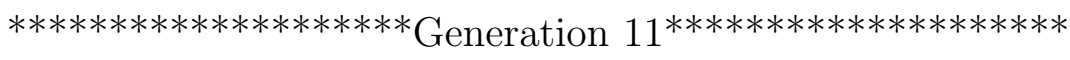

Generation's average fitness: 0.1412690438334024

Generation's best fitness: 0.17893315327481432

Generation's best individual:

$[4,3,3,2,4,2,5,5,7,4,6,6,6,1,3,3,1,0,5,5,1,2,0,4,7,0,0,6,0,7,1,6$, $7,7,2,3,0,3,2,0,3,5,7,1,1,7,7,3,2,5,6,2,5,5,0,7,2,4,2,7,6,7,1,1,5$, $4,3,5,6,5,3,7,2,1,3,2,1,3,2,3,0,1,1,2,7,5,6,5,5,7,2,4,6,7,2,4,4,6$, $6,3,1,1,6,0,0,6,3,6,0,5,4,3,1,1,6,2,7,2,2,7,0,0,4,5,1,5,7,4,5,0,2$, $0,4,0,1,5,0,4,0,0,0,2,1,6,6,1,4,2,0,2,6,5,1,0,0,6,6,1,4,1,0,3,7,5$, $5,3,3,1,4,3,7,1,3,5,4,1,1,6,7,7,0,0,7,7,6,6,6,0,7,7,4,4,1,6,6,5,3$, $4,4,4,1,4,5,7,1,7,6,2,7,3,2,0,7,1,2,4,7,4,5,2,4,3,0,4,6,1,4,7,7,6$, $6,3,5,5,0,1,6,5,0,3,1,6,3,3,0,0,1,0,0,3,6,6,0,0,3,6,4,0,6,4,5,3,7$, $0,2,7,0,0,6,5,5,7,0,5,1,6,3,0,4,6,2,2,3,4,3,4,5,3,6,2,4,7,1,5,4,3$, $7,7,4,0,5,7,6,6,5,6,2,3,1,5,1,5,7,6,7,2,2,4,3,2,0,1,4,2,4,0,7,7,1$, $2,6,5,1,2,6,6,6,5,6,3,2,2,1,6,5,5,4,7,2,7,4,2,0,0,5,3,3,7,6,6,5,1$, $3,4,3,6,4,1,0,7,4,6,2,0,4,2,0,5,1,6,3,3,4,6,7,4,2,3,3,5,1,0,0,1,5$, $7,6,7,1,5,4,4,0,3,5,5,4,4,6,3,2,1,0,6,3,3,6,0,3,7,3,2,4,1,0,4,2,6$, $3,6,2,4,4,1,5,1,6,1,1,5,3,0,0,2,2,3,2,7,3,5,7,2,6,2,5,3,0,1,6,0,6$, $1,0,2,0,0,2,2,1,3,7,3,1,3,7,2,5,6,2,7,4,0,2,1,6,0,4,6,5,6,7,6,7,3$, $7,6,2,1,1,3,7]$

Generation's best community (list of genes):

PIK3R1 H2AFX RAD9A FANCD2 ATF1 ANAPC7 SNRK RAD51AP1 ATG13 SUMO1 RPA2 MRC1 BACH1 E2F1 ATR HIPK3 RPA1 PIAS1 MSH2 BRAP FAM175A MDM2 MDM4 MUS81 C11orf30 TOPBP1 MRE11A RELA MAK EP300 CASP3 SP1 IFT57 PRMT1 NLRP10 CCND1 PRKDC CCNH NRG1 TNFRSF10B GPS2 CNTN2 RECQL5 DEDD2 RNF20 PPARA CDC25C E4F1 KRAS SRY CHGB HTT PPARG RHEBL1 AKT1 TRIM68 PARS2 TIRAP RPL31 HIF1A EME1 CDK1 
Number of genes inside the community: 62

$* * * * * * * * * * * * * * * * * * * *$ Generation $12 * * * * * * * * * * * * * * * * * * * *$

Generation's average fitness: 0.14387642364218087

Generation's best fitness: 0.1874189364461738

Generation's best individual:

$[3,3,2,2,2,5,3,4,4,1,0,7,7,6,2,6,1,2,4,3,6,3,3,0,5,6,2,1,7,2,1,6$, $2,2,7,6,3,1,5,6,0,4,3,2,7,2,0,4,6,4,1,3,6,4,2,3,4,1,7,7,5,6,2,2,2$, $4,0,7,4,6,6,2,5,4,1,7,0,2,0,3,0,3,6,6,6,0,5,1,1,4,1,5,5,4,7,4,7,1$, $6,2,7,6,2,5,1,4,4,3,3,0,0,6,6,6,1,3,2,5,3,5,1,7,4,5,1,5,1,4,6,6,4$, $6,1,3,4,6,2,6,7,1,5,7,1,4,6,2,0,5,5,6,4,2,2,3,0,5,0,1,7,7,3,4,3,4$, $6,2,7,5,6,3,4,7,5,4,6,1,2,4,1,0,4,0,3,4,4,3,2,7,4,2,4,1,1,1,7,3,0$, $1,3,2,7,0,3,4,2,0,7,5,2,7,3,3,1,3,4,4,5,5,7,6,5,2,2,2,1,1,4,7,3,6$, $1,7,7,1,2,7,5,5,5,0,4,3,6,0,5,1,6,7,1,6,1,3,7,3,0,6,1,4,2,7,2,7,1$, $7,4,0,4,5,0,6,3,2,3,5,6,5,2,1,2,1,2,7,3,0,4,5,1,5,1,5,7,0,2,4,6,1$, $2,2,4,1,0,7,0,4,4,5,0,5,7,0,0,0,3,1,4,3,5,2,5,6,4,7,5,0,1,3,3,7,4$, $0,4,7,5,0,1,1,0,7,5,6,7,4,2,6,6,3,7,6,4,1,4,7,1,3,6,2,4,0,6,7,4,4$, $0,6,7,2,3,1,5,1,5,1,3,7,7,3,3,4,2,4,1,0,2,2,3,5,5,3,7,2,3,3,2,3,6$, $0,2,5,5,0,2,5,4,1,2,6,6,4,0,6,7,2,1,6,5,0,2,6,0,0,0,6,7,0,0,4,7,7$, $1,2,4,5,7,1,1,0,5,5,4,2,4,7,5,0,3,6,6,0,2,0,6,2,3,4,5,4,5,7,1,0,6$, $4,2,1,5,4,2,3,7,6,2,3,6,5,2,6,7,3,2,4,4,4,3,2,5,6,2,2,1,1,4,6,2,3$, $5,5,5,3,2,0,7]$

Generation's best community (list of genes):

BRIP1 H2AFX CDK19 FANCD2 RXRB PLAGL1 TGFB1I1 CSTF1 THRB MDC1 BRD7 KAT2B CREBBP VDR ABL1 RPA1 PER3 PPM1L MED1 MSH2 MDM2 TOPBP1 HIST2H2AB GSK3B RELA WHSC1 TRADD HSP90AA1 HSPA5 SP1 WNT2B DCLRE1A STMN1 BCL2L1 HBA1 JUNB XRCC6 PLK1 NR1I2 HIST3H3 BRE SMARCA4 CTBP1 ERCC3 GTF2B MMS19 RXRA UBE3A BCL3 RNF20 XRCC1 HDAC2 DDX17 HDAC1 SLC9A3R2 UBA1 HTT NOD1 UPF1 ERCC6 SHISA5 YWHAH

Number of genes inside the community: 62

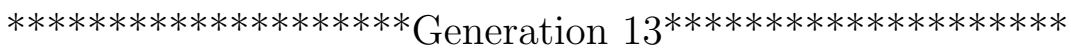

Generation's average fitness: 0.14629853416116823

Generation's best fitness: 0.18

Generation's best individual:

$[0,0,2,4,0,2,2,2,4,7,1,3,3,0,0,4,1,3,0,6,4,6,6,5,4,4,0,7,3,4,0,2$, $0,7,7,0,5,0,6,0,7,0,0,6,4,2,1,7,7,5,2,3,7,1,6,5,4,6,1,5,7,7,0,7,0$, 
$6,3,1,1,0,2,2,1,3,3,6,1,3,7,3,3,3,7,2,6,6,4,0,7,3,6,5,3,0,0,4,4,3$, $6,0,7,1,2,7,4,4,3,5,5,3,6,3,7,1,2,5,5,2,3,0,4,0,5,1,7,7,0,5,3,6,7$, $1,0,0,1,2,6,0,2,2,2,1,2,5,0,3,7,0,6,6,7,1,3,3,0,0,5,4,1,5,4,2,2,1$, $0,7,1,7,4,7,6,7,7,6,4,0,6,5,5,4,2,1,3,1,1,6,4,2,5,1,4,3,1,0,7,0,4$, $4,4,2,0,3,7,5,1,0,2,4,0,3,2,0,2,6,2,1,4,6,5,7,6,4,7,5,2,1,3,0,5,0$, $5,7,7,1,0,3,2,1,6,7,3,0,3,0,4,7,3,1,2,3,5,7,5,7,6,6,5,7,6,0,5,2,3$, $1,2,0,7,7,6,1,0,2,6,3,0,1,2,7,3,2,6,0,0,6,4,3,4,3,7,6,3,0,3,4,1,1$, $2,3,4,6,3,6,5,7,7,4,1,7,3,1,3,1,2,5,5,7,1,0,7,7,4,5,3,3,0,1,0,4,7$, $1,7,1,4,3,2,5,3,1,4,3,1,0,2,5,4,4,3,3,7,6,7,2,7,7,4,6,5,7,2,2,3,3$, $1,6,2,3,7,7,4,5,2,4,4,4,0,6,1,7,3,4,6,1,5,5,0,1,3,5,3,6,2,5,3,4,5$, $4,4,0,4,7,4,3,2,1,6,2,4,3,1,1,6,5,0,3,1,6,5,6,4,3,5,1,2,1,0,3,4,6$, $4,6,2,7,5,6,6,7,3,0,7,0,2,4,5,6,4,6,3,1,5,2,0,7,5,4,5,2,6,6,2,1,1$, $3,6,2,7,4,4,4,2,7,3,3,3,4,7,1,4,5,4,7,0,7,7,3,7,0,0,1,3,0,0,4,5,6$, $7,3,6,0,3,1,0]$

Generation's best community (list of genes):

PALB2 H2AFX BAP1 KBTBD7 VCP RRM2B RAD50 KAT5 SUMO1 E2F1 HIPK3 SQSTM1 RBBP8 PIAS1 UBE2I PLK3 RIPK1 RNF4 HAP1 MOB4 POLR1E PTK2B ESR1 RELA EP300 ULK2 SP1 DCLRE1A CASP7 MED12L LRIF1 PIAS4 UBE2U NR5A2 SMARCA4 CDK7 SMAD3 IKBKG CDC5L JUN MBD3 MAP2K6 TAOK3 RFWD2 DNAJA3 FASLG GTF2F1 ACACB HDAC1 NR0B2 APLP2 CSNK2A2 CLSPN CHGB HIPK2 PPP2R5C ETS1 KPNA2 MED17 NSD1

Number of genes inside the community: 60

************************Generation $14 * * * * * * * * * * * * * * * * * * * *$

Generation's average fitness: 0.14870902320618978

Generation's best fitness: 0.19056486654252017

Generation's best individual:

$[7,0,1,3,6,4,6,4,0,5,2,5,1,5,2,4,7,3,3,0,1,7,7,4,0,6,2,1,6,1,7,6$, $7,5,6,6,6,5,4,2,0,4,6,1,0,2,2,7,0,3,4,3,6,7,2,7,6,5,7,2,2,1,6,0,5$, $4,3,5,6,5,3,7,2,1,3,2,1,3,2,3,0,1,6,6,6,0,5,1,1,4,1,5,5,4,7,4,7,1$, $6,2,7,6,2,5,1,4,4,3,3,0,0,6,6,6,1,3,2,5,3,5,1,7,4,5,1,5,1,4,6,6,4$, $6,1,3,4,6,2,6,7,1,5,7,1,4,6,2,0,5,4,4,6,2,0,0,6,2,2,1,0,4,5,6,3,3$, $2,2,7,0,5,7,0,0,6,6,3,5,3,4,2,3,4,4,6,3,5,3,2,3,5,1,4,3,1,0,7,0,4$, $4,4,2,0,3,7,5,1,0,2,4,0,3,2,0,2,6,2,1,4,6,5,7,6,4,7,5,2,1,3,0,5,0$, $5,7,7,1,0,3,2,1,6,7,3,0,3,0,4,7,3,1,2,3,5,7,5,7,6,6,5,7,6,0,5,2,3$, $1,2,0,7,7,6,1,0,2,6,3,0,1,2,7,3,2,6,0,0,6,4,3,4,3,7,6,3,0,3,4,1,1$, $2,3,4,6,3,6,5,7,7,4,1,7,3,1,3,1,2,5,5,7,1,0,7,7,4,5,3,3,0,1,0,4,7$, 
$1,7,1,4,3,2,5,3,1,4,3,1,0,2,5,4,4,3,3,7,6,7,2,7,7,4,6,5,7,2,2,3,3$, $1,6,2,3,7,7,4,5,2,4,4,4,0,6,1,7,3,4,6,1,5,5,0,1,3,5,3,6,2,5,3,4,5$, $4,4,0,4,7,4,3,2,1,6,2,4,3,1,1,6,5,0,3,1,6,5,6,4,3,5,1,2,1,0,3,4,6$, $4,6,2,7,5,6,6,7,3,0,7,0,2,4,5,6,3,4,2,7,6,3,5,0,5,5,7,5,6,4,2,4,1$, $7,2,0,2,5,0,3,4,6,0,2,2,3,1,1,2,3,3,4,6,1,0,4,0,5,4,1,5,7,2,1,4,5$, $1,4,0,4,4,3,3]$

Generation's best community (list of genes):

SHFM1 RAD52 RAD9A CDK19 FKBP5 ATF1 TOPORS ATG13 SUMO1 RPA2 THRB MDC1 BRD7 KAT2B CREBBP VDR ABL1 RPA1 PER3 PPM1L MED1 MSH2 MDM2 ESR1 RELA EP300 ULK2 SP1 DCLRE1A CASP7 MED12L LRIF1 PIAS4 UBE2U NR5A2 SMARCA4 CDK7 SMAD3 IKBKG CDC5L JUN MBD3 MAP2K6 TAOK3 RFWD2 DNAJA3 FASLG GTF2F1 ACACB HDAC1 NR0B2 APLP2 CSNK2A2 CLSPN CHGB ETS1 AATF KPNA2 VRK1 MED17 FBXO11 FAIM2

Number of genes inside the community: 62

********************* Generation $15 * * * * * * * * * * * * * * * * * * * *$

Generation's average fitness: 0.1511111573474144

Generation's best fitness: 0.19302775984506132

Generation's best individual:

$[3,6,1,2,2,5,2,3,5,4,0,6,0,0,7,2,5,0,0,2,6,6,3,5,7,2,7,4,3,0,1,0$, $6,6,2,3,4,5,4,6,1,1,5,7,0,6,6,6,6,5,5,2,5,1,1,3,7,1,2,1,4,4,5,6,2$, $4,7,5,5,5,6,0,4,6,7,5,1,5,0,1,2,3,3,0,2,4,6,0,1,0,5,4,3,4,6,2,2,5$, $5,7,4,1,2,4,1,1,6,2,3,3,7,0,7,1,5,2,2,0,5,3,5,2,3,4,4,2,7,6,1,5,3$, $7,3,5,1,7,2,6,4,5,6,1,4,7,4,3,4,0,3,3,7,0,5,2,2,1,2,2,4,1,5,2,2,1$, $3,3,6,6,0,2,1,0,2,7,2,7,6,1,5,3,1,1,2,2,7,6,2,5,5,6,0,7,5,2,6,3,2$, $0,5,4,0,4,0,6,1,7,4,3,5,7,2,1,4,1,4,3,3,2,3,7,5,7,6,4,7,1,5,1,2,5$, $1,3,0,2,5,2,5,4,1,7,5,7,0,2,4,4,6,5,0,1,6,4,5,3,4,5,4,2,0,0,7,6,1$, $1,1,3,1,6,6,1,3,3,3,5,4,5,4,3,4,1,4,4,7,4,7,6,3,0,1,4,3,0,1,5,0,5$, $5,4,2,7,5,2,3,0,5,7,7,0,0,1,2,1,0,3,6,4,3,5,6,0,3,2,2,1,1,5,7,5,0$, $0,0,4,0,4,5,7,5,7,1,4,3,6,4,2,2,2,6,1,0,6,7,4,0,7,3,6,2,5,0,2,5,1$, $4,2,5,7,7,7,7,6,0,6,5,4,6,2,6,5,2,7,1,3,7,7,7,7,0,0,6,5,5,4,5,1,0$, $5,7,3,5,5,4,0,1,3,5,4,2,7,6,1,4,1,4,4,1,6,4,7,1,5,2,6,5,4,3,6,2,2$, $6,2,2,1,2,1,7,1,6,0,2,2,7,5,3,2,5,1,6,7,5,3,2,6,4,2,2,2,4,2,6,2,1$, $3,0,2,7,7,0,4,2,1,3,1,3,4,7,1,4,5,4,3,0,7,7,5,7,0,5,1,3,0,0,4,3,6$, $6,3,6,0,3,2,0]$

Generation's best community (list of genes):

SHFM1 FANCD2 RECQL4 AP1B1 KBTBD7 DMC1 TGFB1I1 RAD51D SUMO1 
ING5 MDC1 E2F1 CREBBP TFAP2A HIPK3 NUFIP1 PIAS1 UBE2I TERF2 MDM4 RNF4 FEZ1 MSH6 UIMC1 MOB4 EP300 BCCIP CASP3 SP1 YLPM1 WNT2B CHD3 WDR16 XRCC6 LRIF1 ACACA SETDB1 PIAS4 NR1I2 BRE PRKDC SMAD3 IKBKG CHEK1 GTF2B NR3C1 BUB1B DEDD2 DDX17 E4F1 DGKZ APLP2 SRY CSNK2A2 RARA ETS2 HTT PPARG DARS ETS1 PPP2R5E TP63 KPNA2 MED17

Number of genes inside the community: 64

********************** Generation 16 * $* * * * * * * * * * * * * * * * * * * *$

Generation's average fitness: 0.15366877405510673

Generation's best fitness: 0.19961612284069097

Generation's best individual:

$[5,7,7,3,1,7,7,7,2,4,6,1,4,0,4,6,2,7,2,5,3,5,3,6,2,2,3,1,4,3,1,4$, $0,0,7,3,2,5,5,4,0,5,0,3,1,4,2,6,5,0,4,1,2,3,5,3,0,3,0,3,4,1,4,7,2$, $1,6,4,0,7,7,2,1,0,5,0,1,1,6,7,5,4,1,7,4,3,6,2,1,4,4,4,0,0,3,6,3,1$, $7,3,3,1,0,5,1,2,4,0,1,0,0,6,1,6,1,3,2,1,0,5,1,7,5,5,1,5,1,4,1,0,4$, $6,7,7,4,6,2,7,7,1,7,7,5,4,6,2,6,5,4,6,4,2,0,7,6,1,2,1,0,4,5,6,5,3$, $2,0,7,4,0,5,0,5,0,6,3,2,3,4,2,3,4,4,6,3,5,3,2,3,4,6,5,3,6,4,3,5,0$, $7,5,2,3,3,5,7,2,5,0,6,5,3,4,7,3,7,2,5,4,6,7,2,4,7,6,7,5,1,4,5,2,7$, $1,4,3,6,0,0,2,2,3,7,2,7,6,5,6,0,3,2,5,6,1,3,4,2,2,7,1,4,7,3,0,1,5$, $5,5,0,6,0,3,1,5,3,4,6,0,0,6,0,3,6,6,7,1,7,6,6,4,7,3,1,5,4,0,7,4,1$, $6,7,6,5,4,5,1,7,1,6,2,7,4,1,4,2,6,3,6,3,7,0,7,0,0,6,4,4,6,6,4,5,2$, $4,6,4,5,5,4,3,6,5,2,3,0,5,1,5,7,2,4,3,7,1,4,1,1,1,5,4,4,4,4,2,1,0$, $7,6,6,6,5,5,7,2,5,1,0,2,0,2,2,5,4,0,1,7,6,4,2,2,3,3,0,2,0,2,0,4,4$, $7,0,7,3,6,2,5,3,1,7,4,6,7,1,2,7,3,1,1,5,7,5,4,3,4,4,3,4,4,4,3,4,0$, $1,2,2,1,2,0,7,1,5,7,4,2,3,6,6,3,0,6,2,4,3,7,2,0,1,2,7,4,6,7,7,6,1$, $0,5,5,5,6,7,2,3,3,7,0,2,3,5,1,7,1,3,3,7,2,5,0,3,5,2,3,6,1,2,4,1,0$, $5,6,4,7,1,4,1]$

Generation's best community (list of genes):

ATAD2 NBN CDK19 FANCD2 ANAPC7 BLM TOPORS FADD KAT5 SUMO1 KAT7 MRC1 MDC1 KAT2B E2F1 CREBBP PPP1R13L ATR VDR XRCC5 ABL1 RPA1 PER3 NUFIP1 MED1 TERF2 MDM2 SP1 WNT2B HBA1 JUNB GABRB1 PIAS4 NCL NR1H3 SMARCA4 ADAP1 PPM1B SMAD3 RECQL5 UBE3A ZMIZ1 BCL3 SMAD6 SLC9A3 HDAC2 DDX17 HDAC1 NR0B2 SLC9A3R2 SERPINH1 UBA1 ETS2 PPARG BID ETS1 KPNA2 TICAM1 YWHAH TOP2A CDK1 PDGFRB

Number of genes inside the community: 62

***********************) Generation $17 * * * * * * * * * * * * * * * * * * * *$

Generation's average fitness: 0.1560841262853778 
Generation's best fitness: 0.19627966645285438

Generation's best individual:

$[3,3,2,2,2,5,3,4,4,1,0,7,7,6,2,6,1,2,4,3,6,3,3,0,5,6,4,4,3,4,1,7$, $1,5,3,3,3,3,3,5,6,0,7,0,6,2,2,3,0,0,5,1,3,7,5,4,2,6,1,6,7,0,5,0,6$, $5,3,2,2,1,6,3,7,6,4,7,1,1,0,1,7,1,6,2,6,0,0,2,1,4,6,5,4,5,2,3,4,0$, $6,3,3,1,4,3,4,4,0,4,3,7,2,7,0,7,2,0,6,0,5,2,6,5,3,7,6,4,4,6,2,7,5$, $5,7,5,1,6,4,1,7,4,1,1,0,5,1,6,6,4,2,3,1,2,6,1,2,7,4,4,5,4,0,2,5,1$, $7,6,5,4,4,1,6,6,2,7,6,3,0,7,3,0,6,7,0,2,1,6,4,2,5,1,4,3,1,0,7,0,4$, $4,4,2,0,3,7,5,1,0,2,4,0,3,2,0,2,6,2,1,4,6,5,7,6,4,7,1,4,1,3,7,5,1$, $7,4,6,0,5,2,2,2,3,6,2,4,5,0,1,3,0,2,5,6,6,7,5,3,6,0,1,4,7,1,0,4,7$, $7,2,7,3,7,3,5,1,7,7,1,1,2,4,0,2,6,3,1,1,3,3,1,3,7,1,7,1,3,1,4,2,7$, $0,4,6,4,2,4,1,4,0,1,4,6,5,2,6,3,5,0,1,7,1,4,3,7,0,7,7,7,3,4,7,1,6$, $5,6,2,5,6,4,3,1,4,1,0,2,3,4,7,6,2,2,6,4,0,1,3,4,3,0,4,7,2,2,0,3,2$, $5,2,5,0,7,6,5,3,1,1,1,2,0,2,2,5,0,0,4,7,0,7,4,4,6,5,5,6,2,7,2,2,5$, $5,6,7,5,7,7,4,7,1,1,7,7,1,2,4,4,5,0,4,0,2,3,4,4,3,4,2,6,6,2,3,2,4$, $2,2,4,2,6,3,6,2,3,3,5,5,7,3,6,6,2,3,3,6,4,3,0,7,1,2,6,6,7,3,0,7,0$, $2,3,5,6,3,1,2,2,3,4,7,6,5,5,1,7,7,0,6,2,1,5,5,7,3,5,4,7,5,2,5,1,6$, $2,2,4,5,1,7,5]$

Generation's best community (list of genes):

BRIP1 H2AFX FANCD2 STK11IP BLM VCP ATRIP SUMO1 KAT7 ING5 RPA2 MDC1 E2F1 PIAS1 IFI16 NTHL1 UBE2I TP53BP1 ETV5 CASP1 RNF4 NFYA PTK2B ESR1 RELA EP300 ULK2 ZNF280B SP1 MCPH1 ING4 JUNB HUWE1 RB1 ANG CCND1 POU2F1 NCL MAP4K4 BRE TIPARP PRKDC ADAP1 PTS CDC14B CDC5L APOL2 NKX2-1 NR3C1 PA2G4 ERCC2 HDAC2 RALBP1 HDAC1 ULK1 BAK1 BID RAD17 KPNA2 VRK1 TOP2A CDK1

Number of genes inside the community: 62

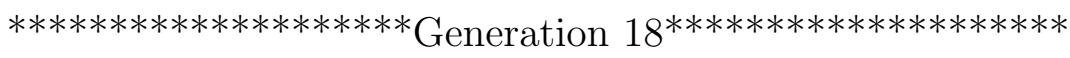

Generation's average fitness: 0.1585739672687739

Generation's best fitness: 0.1974479516453996

Generation's best individual:

$[6,2,3,7,4,4,4,3,0,2,5,3,3,5,4,0,1,7,3,6,2,7,2,6,2,2,7,3,4,4,1,3$, $4,1,5,4,7,6,0,6,2,0,2,0,5,2,0,2,2,6,2,6,4,3,4,5,6,1,3,0,2,1,3,3,6$, $5,2,6,1,1,2,0,3,6,7,3,1,7,3,5,2,3,5,5,0,4,3,0,6,7,6,4,5,1,5,5,6,0$, $2,6,4,1,1,4,7,5,3,6,7,3,4,0,1,3,6,1,6,7,2,1,4,3,4,0,0,1,6,7,2,6,2$, $5,3,2,6,2,3,4,0,0,0,2,1,6,6,1,4,2,0,2,6,5,1,0,0,6,6,1,4,1,0,3,7,5$, $5,3,3,1,4,3,7,1,3,5,4,1,1,6,7,7,0,0,7,3,7,7,0,0,5,3,1,2,3,1,7,2,6$, 
$4,5,4,5,7,2,0,1,0,3,5,7,0,0,3,7,1,1,5,3,7,6,4,7,3,7,4,4,0,3,0,4,5$, $6,5,5,3,5,4,6,0,3,1,0,4,6,1,0,3,6,7,6,7,1,7,2,6,0,7,7,7,6,0,2,3,1$, $2,7,4,4,6,6,0,1,1,7,7,1,2,4,5,2,6,3,1,0,6,3,0,3,7,1,4,1,3,1,7,2,7$, $0,4,5,4,2,4,2,4,7,1,1,6,5,4,6,7,7,3,1,7,1,4,2,7,0,0,7,7,5,4,6,2,6$, $5,3,6,5,4,1,3,5,4,0,7,7,3,4,7,6,2,2,5,7,6,7,3,2,2,6,6,5,7,4,5,7,5$, $1,5,2,6,6,3,3,7,5,2,2,0,5,0,7,0,7,1,1,6,3,1,4,0,4,4,2,5,2,4,0,1,2$, $7,4,7,3,6,0,5,0,1,5,4,7,2,0,7,5,7,5,6,1,7,1,6,1,5,0,7,0,4,2,7,3,0$, $1,7,4,4,2,7,5,2,6,5,1,3,0,4,2,7,0,5,2,4,1,3,1,1,6,4,3,5,3,7,0,3,2$, $2,2,3,7,4,5,6,7,6,3,4,3,6,1,3,5,4,3,3,6,0,7,6,2,6,6,5,3,2,3,4,1,2$, $7,3,3,6,7,6,0]$

Generation's best community (list of genes):

H2AFX FANCD2 WRN TGFB1I1 TOPORS RAD50 ATRIP SUMO1 DBNL E2F1 CUL3 ATR GINS2 ING1 FOXO3 MSH2 BRAP FAM175A MDM2 MDM4 MUS81 C11orf30 TOPBP1 MRE11A DDX5 WHSC1 EP300 CASP3 RYBP TIMM13 HMG20B HBA1 XRCC6 RB1 ESRRA CCND1 POU2F1 BRE TIPARP PRKDC PTS CDK7 CDC14B CDC5L MMS19 DNAJA3 PPP2R5B DDX17 ZNF350 E4F1 HDAC1 CSNK2A2 E2F4 RARA UBA1 AKT1 RAD54L2 MTA1 RBBP4 AATF TOP2A

Number of genes inside the community: 61

$* * * * * * * * * * * * * * * * * * * *$ Generation $19 * * * * * * * * * * * * * * * * * * * *$

Generation's average fitness: 0.16144249532069577

Generation's best fitness: 0.19702842377260982

Generation's best individual:

$[0,0,3,6,3,6,0,2,5,0,3,1,4,7,2,3,1,4,5,5,1,6,0,2,4,6,4,7,6,7,1,0$, $2,7,2,2,0,1,0,7,6,5,3,3,0,6,1,5,6,3,4,1,3,3,4,4,0,5,7,2,2,1,6,0,5$, $4,3,5,6,5,3,7,2,1,3,2,1,3,5,6,0,3,6,6,6,0,5,1,1,4,1,5,5,4,7,4,7,1$, $6,2,7,6,2,5,1,4,4,3,3,0,0,6,6,6,1,3,2,5,3,5,1,7,4,5,1,5,1,4,1,2,4$, $7,3,0,5,7,3,0,0,2,5,4,5,4,4,1,3,7,4,0,0,6,6,3,1,5,3,1,0,1,4,0,5,5$, $5,5,3,6,2,6,6,0,5,1,3,0,7,1,2,5,2,7,3,6,3,5,6,6,0,7,0,2,4,2,5,3,2$, $5,6,4,4,6,2,5,1,6,5,6,6,6,5,2,5,1,0,0,4,5,7,4,2,7,4,6,5,6,5,5,1,7$, $5,6,5,2,3,7,7,7,0,0,5,0,6,1,7,4,1,3,1,6,0,5,3,2,6,0,0,6,2,2,1,2,6$, $4,3,6,5,7,1,2,1,0,2,5,1,4,3,6,3,2,1,4,4,4,4,5,4,4,1,2,3,4,1,7,5,4$, $0,7,6,7,2,5,4,3,0,5,7,6,3,1,2,0,0,4,3,1,5,7,7,0,4,0,4,5,7,4,7,4,6$, $2,6,6,7,7,2,6,4,4,1,5,4,7,6,3,6,6,1,5,5,1,2,1,4,5,0,3,2,6,7,1,5,6$, $7,1,4,5,0,5,1,4,6,4,3,2,4,4,0,2,4,2,4,5,4,3,3,3,4,1,0,6,6,3,7,4,5$, $2,5,7,6,2,5,2,4,3,0,7,5,5,2,3,0,6,6,7,3,2,0,7,2,5,3,2,2,3,3,5,7,3$, $6,5,2,0,5,0,3,1,2,5,1,3,6,7,5,6,4,6,3,1,0,0,0,2,0,6,4,0,2,4,4,1,5$, 
$6,5,4,0,4,6,2,7,4,1,1,5,7,0,7,3,2,6,3,2,6,1,2,0,2,0,0,5,3,3,2,1,6$, $7,3,4,1,1,6,6]$

Generation's best community (list of genes):

NBN H2AFX RAD9A FANCD2 RXRB BAP1 BLM TOPORS ATG13 SUMO1 THRB MDC1 BRD7 KAT2B CREBBP VDR ABL1 RPA1 PER3 NUFIP1 BRAP ATN1 MDM2 MDM4 TNFRSF10A MSH6 EP300 CASP3 BCL2 HMG20B NLRP10 BCL2L1 MSH3 TOP3A RB1 CCND1 RCHY1 BRE PRKDC SMAD3 NR1H4 NR3C1 MYCBPAP UBE3A ZMIZ1 HMGB1 UBE2D3 ATG12 GSK3A PPARG AKT1 HIPK2 PPP2R5C PPP2R5D TP63 AXL TOP2A EME1 CDK1

Number of genes inside the community: 59

$* * * * * * * * * * * * * * * * * * * *$ Generation $20 * * * * * * * * * * * * * * * * * * * *$

Generation's average fitness: 0.16426696559494613

Generation's best fitness: 0.20320855614973263

Generation's best individual:

$[5,0,2,5,7,2,2,2,3,2,3,1,4,7,2,3,1,4,5,5,1,6,0,2,4,6,4,7,6,7,1,0$, $2,7,2,2,3,7,7,5,3,0,0,4,4,7,0,5,3,4,6,2,3,7,5,6,0,5,7,2,3,5,6,2,2$, $2,6,7,3,2,4,5,6,0,3,1,1,4,1,1,5,7,4,5,2,7,6,5,1,3,4,1,0,0,5,3,6,3$, $3,0,4,1,5,4,4,1,1,4,6,4,3,0,1,6,1,3,2,5,3,5,1,7,4,5,1,5,1,4,6,6,4$, $6,1,3,4,6,2,6,7,1,5,7,1,4,6,2,0,5,4,4,6,2,0,0,6,2,2,1,0,4,5,6,3,3$, $2,2,7,0,5,7,1,0,5,7,6,7,6,1,5,3,1,1,2,2,7,4,2,5,0,6,0,7,5,2,6,3,7$, $0,5,4,0,4,0,6,1,7,4,3,5,7,2,0,4,1,4,3,3,2,3,7,5,7,6,4,7,1,5,1,1,5$, $5,3,0,2,5,2,5,4,1,7,5,7,0,2,4,4,6,5,0,1,6,4,0,3,4,5,4,2,2,4,5,6,3$, $4,2,3,4,0,0,2,1,6,4,4,5,5,6,5,2,4,7,6,4,4,3,3,1,4,7,1,7,6,1,6,3,5$, $2,6,3,3,6,7,3,6,4,6,0,7,1,1,4,1,1,0,0,1,0,3,4,5,2,0,0,5,7,1,4,3,6$, $5,4,5,6,2,7,3,6,5,1,0,0,7,7,0,7,6,5,1,3,7,1,2,1,7,1,7,7,0,0,1,0,6$, $2,1,7,0,2,4,5,2,0,2,5,7,2,3,7,4,7,1,3,5,6,3,0,3,2,7,3,5,1,5,6,6,0$, $7,7,6,6,0,5,3,2,1,4,4,7,3,7,0,3,3,1,1,4,5,1,3,6,6,4,4,2,4,2,0,5,7$, $4,4,5,5,7,4,4,3,6,5,0,3,7,2,4,1,6,6,4,0,7,5,1,2,2,2,0,2,7,4,3,3,7$, $4,2,0,6,6,5,5,0,2,5,3,0,2,0,1,2,2,3,6,0,6,4,1,6,4,7,3,5,2,6,5,0,3$, $7,2,0,7,1,1,4]$

Generation's best community (list of genes):

NBN H2AFX RAD9A FANCD2 ESRRB SUMO1 MAST3 ING5 MDC1 HRAS E2F1 TFAP2A ATMIN ATR VDR ABL1 RPA1 PER3 PPM1L MED1 MSH2 MDM2 FEZ1 MSH6 UIMC1 MOB4 EP300 CASP3 SP1 YLPM1 BCL2 CHD3 WDR16 RB1 HIST3H3 NR1H3 PRKDC CCNH SMAD3 IKBKG PEBP1 NR1H4 JUN NR3C1 BUB1B PA2G4 BCL3 XIAP HMGB1 UBE2D3 PPP2R5B CDC25C HDAC1 SLC9A3R2 


\section{SERPINH1 E2F4 DAXX MTA1 KPNA2 HIF1A CDK1 NSD1}

Number of genes inside the community: 62

\section{A.2 Generations 291 to 300}

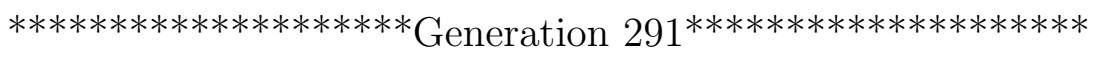

Generation's average fitness: 0.5431339581075925

Generation's best fitness: 0.55404699738903395

Generation's best individual:

$[5,0,2,5,7,2,2,2,3,7,7,1,4,7,2,3,1,4,5,5,1,6,0,2,4,6,4,7,6,4,1,2$, $7,1,6,4,7,3,0,2,2,0,2,0,5,2,0,2,5,0,4,1,0,5,4,2,0,3,0,4,7,5,6,7,0$, $6,2,6,1,3,4,7,1,0,6,0,1,4,4,2,7,1,6,2,6,0,0,2,1,3,4,4,0,0,3,6,3,1$, $7,3,3,1,1,5,1,3,4,3,3,0,0,6,1,2,3,6,5,1,4,1,1,5,7,3,1,6,4,4,6,6,4$, $6,4,0,1,5,0,4,5,5,7,1,1,3,1,2,0,5,2,0,5,7,3,4,4,5,2,1,7,3,2,6,5,6$, $4,0,2,3,2,5,4,0,6,2,0,6,1,1,2,6,4,0,3,0,4,4,3,6,5,1,4,3,1,0,7,0,4$, $0,5,4,0,3,4,5,1,2,2,4,3,1,3,6,3,6,1,6,2,6,5,2,4,4,7,5,7,1,3,7,0,1$, $7,5,2,6,7,2,0,2,1,5,3,6,4,2,1,0,0,4,7,4,4,4,5,4,3,4,7,2,6,6,7,3,1$, $3,2,0,7,7,0,1,1,5,5,7,1,4,0,6,3,1,6,1,1,7,3,7,6,7,3,0,6,7,1,1,5,1$, $0,3,7,6,0,0,7,3,2,5,4,3,2,1,3,2,7,5,4,4,7,0,1,0,3,6,0,1,7,1,4,3,6$, $5,4,5,6,6,7,3,7,4,1,3,3,5,1,5,0,5,4,4,3,0,5,6,5,0,0,3,5,5,2,1,2,0$, $2,5,2,6,2,3,5,3,5,1,0,2,7,2,2,5,7,7,1,3,7,7,7,6,7,6,7,3,3,2,3,2,7$, $7,2,6,5,0,2,5,2,1,2,6,6,3,0,0,3,3,5,0,6,0,5,3,6,6,4,2,5,0,7,7,7,6$, $0,6,5,5,3,0,2,3,4,6,4,6,3,2,3,1,0,3,4,1,2,2,0,1,7,1,1,2,4,6,5,2,1$, $3,2,6,3,7,3,7,5,5,4,2,7,4,0,1,4,5,4,0,1,7,6,1,4,4,2,0,7,5,2,5,1,6$, $2,2,0,5,1,5,0]$

Generation's best community (list of genes):

NBN H2AFX RAD9A FANCD2 WRN BLM RAD50 KAT5 SUMO1 RPA2 MDC1 KAT2B E2F1 CUL3 CREBBP ATR XRCC5 ING1 ABL1 RPA1 PIAS1 UBE2I MSH2 TP53BP1 MDM2 MRE11A MSH6 ESR1 RELA EP300 SUMO2 RYBP SP1 MCPH1 CHD3 ING4 XRCC6 PIAS4 RB1 CCND1 NR1I2 POU2F1 NCL PRKDC DCLRE1C SMARCA4 SMAD3 NPAS2 CHEK1 JUN NR3C1 RECQL5 HMGB1 HDAC2 DDX17 HDAC1 DAXX HIPK2 RBBP4 STAT5A PCNA ETS1 KPNA2 PGR HIF1A TOP2A CDK1

Number of genes inside the community: 67

**********************Generation 292 2**********************

Generation's average fitness: 0.54278249459636606 
Generation's best fitness: 0.5527272727272727

Generation's best individual:

$[5,0,2,2,2,5,3,4,3,4,3,1,4,7,1,3,1,6,5,5,1,0,0,2,3,6,4,7,6,4,1,7$, $4,1,5,4,7,3,3,2,2,0,2,0,5,2,0,2,5,3,4,1,2,3,5,3,0,3,0,4,7,7,0,7,0$, $0,4,1,1,4,7,2,1,0,6,0,1,4,4,2,2,1,6,2,0,0,2,2,1,3,4,4,0,4,3,6,3,1$, $7,3,3,1,0,5,1,3,4,0,4,0,0,6,1,7,3,6,5,1,7,1,1,0,7,3,1,6,6,4,6,0,6$, $6,4,0,1,5,0,4,5,5,7,1,1,5,1,3,0,5,2,0,5,4,3,4,4,7,2,1,7,3,2,3,5,6$, $4,0,2,3,2,5,4,0,6,2,0,6,1,1,2,3,3,7,3,0,3,4,3,5,5,1,4,3,1,0,7,0,4$, $0,5,4,0,3,1,3,1,2,2,4,3,1,3,6,3,6,4,6,1,6,5,7,6,4,2,4,6,1,3,7,0,1$, $7,6,0,2,3,5,0,2,1,5,3,6,4,6,7,0,7,4,7,7,6,0,4,5,4,7,7,2,6,3,4,7,1$, $3,2,0,7,7,0,1,1,5,6,7,1,4,0,6,3,7,6,1,1,7,3,7,6,7,3,4,6,2,1,1,5,1$, $0,3,7,6,0,0,7,2,0,2,7,3,2,1,6,5,4,5,4,2,4,0,0,0,3,4,5,1,7,1,4,3,6$, $4,0,2,5,6,7,0,6,4,1,3,3,6,1,5,0,5,4,4,3,0,5,6,5,0,2,3,5,5,2,1,3,5$, $4,2,3,6,1,3,5,3,3,1,6,6,7,5,0,3,4,0,1,4,7,7,7,6,4,6,7,5,3,2,3,2,7$, $7,7,5,5,1,7,3,2,1,5,4,7,3,2,4,4,3,5,2,6,7,5,6,4,3,3,4,5,0,3,0,7,7$, $4,6,5,5,3,0,2,3,4,5,7,3,7,2,4,1,6,6,5,1,6,0,0,1,7,1,1,3,4,6,5,2,1$, $4,3,7,3,7,3,7,5,5,4,2,7,0,0,1,1,7,4,2,1,3,6,1,4,4,2,0,7,2,4,0,1,6$, $2,2,3,5,1,7,5]$

Generation's best community (list of genes):

NBN LRIT3 H2AFX RAD9A FANCD2 WRN BLM RRM2B RAD50 KAT5 SUMO1 RPA2 MDC1 KAT2B E2F1 CREBBP ATR XRCC5 ING1 ABL1 RPA1 PIAS1 UBE2I MSH2 TP53BP1 MDM2 MRE11A MSH6 ESR1 RELA NOL3 EP300 SUMO2 GIT1 SP1 MCPH1 CHD3 XRCC6 PIAS4 RB1 CCND1 POU2F1 NCL PRKDC DCLRE1C SMARCA4 SMAD3 CHEK1 JUN NR3C1 RECQL5 HMGB1 PCYT1A HDAC2 DDX17 UBE2L3 HDAC1 DAXX HIPK2 RBBP4 STAT5A PCNA ETS1 KPNA2 TP53INP1 PGR HIF1A TOP2A CDK1

Number of genes inside the community: 69

**********************Generation 293 * $* * * * * * * * * * * * * * * * * * * *$

Generation's average fitness: 0.54337012377776394

Generation's best fitness: 0.5489473684210526

Generation's best individual:

$[5,0,2,5,4,2,2,2,3,2,3,1,4,7,2,3,1,4,5,5,1,6,0,4,4,6,4,7,6,4,1,2$, $4,1,5,4,7,6,0,5,2,0,2,0,5,2,0,2,5,0,4,1,0,3,5,5,6,5,0,4,7,7,0,3,6$, $5,2,6,1,3,4,7,1,0,3,6,1,4,4,2,7,1,6,2,6,0,0,2,1,3,4,4,0,0,3,6,3,1$, $7,3,3,1,0,5,1,3,4,3,3,0,0,6,1,4,4,3,4,1,4,1,1,5,5,6,1,6,4,3,3,6,4$, $6,4,0,1,5,0,4,5,5,7,1,1,7,1,2,3,5,2,0,5,7,3,4,4,7,2,1,7,3,2,3,5,6$, 
4, 0, 2, 3, 2, 5, 4, 0, 6, 2, 0, 6, 1, 1, 2, 6, 4, 0, 3, 0, 4, 4, 3, 6, 5, 1, 4, 3, 1, 0, 7, 0, 4, $0,5,4,0,3,4,5,1,2,2,4,3,1,3,6,3,6,4,6,2,6,5,2,5,7,6,4,7,1,3,7,0,1$, $7,6,2,6,7,5,7,2,1,5,3,6,4,6,0,0,7,7,7,4,6,0,4,5,4,7,6,5,6,3,4,1,1$, $3,2,0,7,7,0,1,1,5,5,7,1,4,0,6,3,7,6,1,1,7,3,7,6,7,3,0,6,7,1,1,5,1$, $0,3,7,6,4,0,7,2,0,2,7,3,2,1,3,2,4,5,4,2,7,0,0,0,3,6,5,1,7,1,4,3,6$, $5,7,6,6,2,6,2,2,4,1,3,3,5,1,5,0,5,4,4,3,0,5,6,5,0,2,3,5,5,2,1,2,1$, $7,2,2,4,2,3,5,3,3,1,6,6,7,5,5,4,7,7,1,0,7,4,7,6,7,6,7,2,6,6,2,3,6$, $0,2,5,6,0,4,5,4,1,2,0,7,3,7,0,3,3,5,0,6,0,5,3,6,6,4,2,5,0,3,0,5,5$, $6,2,5,3,7,4,6,0,4,3,7,3,3,3,6,1,6,3,4,1,6,0,0,1,7,1,1,2,4,4,3,2,1$, $3,2,6,3,7,3,7,5,5,4,2,6,5,5,1,4,5,4,2,1,3,6,1,4,6,4,0,7,3,6,5,1,6$, $2,2,4,5,1,7,4]$

Generation's best community (list of genes):

NBN H2AFX RAD9A FANCD2 WRN BLM RAD50 KAT5 SUMO1 RPA2 MDC1 KAT2B E2F1 CREBBP ATR XRCC5 ING1 ABL1 RPA1 PIAS1 UBE2I MSH2 TP53BP1 MDM2 MRE11A MSH6 ESR1 RELA EP300 SUMO2 SP1 MCPH1 CHD3 GABRB1 XRCC6 PIAS4 RB1 CCND1 POU2F1 NCL PRKDC DCLRE1C SMARCA4 SMAD3 CHEK1 JUN NR3C1 RECQL5 HMGB1 DEDD2 HDAC2 DDX17 HDAC1 DAXX HIPK2 RBBP4 STAT5A PCNA ETS1 KPNA2 PGR HIF1A TOP2A CDK1

Number of genes inside the community: 64

********************** Generation $294 * * * * * * * * * * * * * * * * * * * * *$

Generation's average fitness: 0.54259412369513204

Generation's best fitness: 0.55170603674540685

Generation's best individual:

$[5,0,2,5,7,2,2,2,3,2,2,1,4,7,2,3,1,4,5,5,1,6,0,2,4,6,4,7,6,7,1,3$, $4,1,6,4,6,6,0,5,2,0,2,0,5,2,0,2,5,0,4,1,0,3,4,5,0,3,0,3,7,3,3,3,6$, $5,2,6,1,4,3,7,1,0,6,0,1,4,4,2,4,1,6,2,1,0,0,2,1,3,0,4,0,0,3,6,3,1$, $7,3,3,1,0,5,1,3,4,3,3,0,0,6,1,4,4,3,4,1,7,1,1,3,4,6,1,6,4,4,6,6,4$, $4,4,0,1,5,0,4,5,5,7,1,1,0,1,2,0,5,2,0,5,7,3,4,4,7,2,1,7,3,2,3,5,6$, $4,0,2,3,2,5,4,0,6,2,0,6,1,1,2,6,4,0,3,0,4,4,3,6,5,1,4,3,1,0,7,0,4$, $0,5,2,0,3,4,5,1,2,2,4,3,1,3,6,3,6,4,6,2,6,5,2,5,7,7,4,4,1,3,7,0,1$, $7,6,0,2,5,5,2,2,1,5,3,6,4,6,0,0,0,4,7,4,4,4,5,4,3,4,7,2,6,6,7,3,1$, $3,2,0,7,7,0,1,1,5,5,7,1,4,0,6,3,7,6,1,1,7,3,7,6,7,3,0,6,7,1,1,5,1$, $0,3,7,6,0,0,7,3,2,5,4,3,2,1,3,4,7,5,4,2,7,4,5,2,7,6,0,1,3,1,4,3,6$, $4,0,5,3,0,2,2,2,4,1,3,3,5,1,5,0,5,4,4,3,0,5,6,5,0,0,3,3,4,2,1,3,0$, $2,5,0,4,2,3,5,3,3,1,6,6,7,5,5,3,4,0,1,7,6,4,2,5,5,3,7,2,0,0,2,3,7$, $7,7,3,5,0,5,3,4,1,2,4,6,3,0,2,4,4,3,0,6,7,3,6,6,0,7,3,2,4,2,0,5,7$, 
$4,4,5,5,7,4,6,0,4,3,6,3,3,3,6,1,6,6,2,1,6,0,5,1,7,1,1,2,4,6,1,3,1$, $3,0,7,3,7,3,7,2,7,4,7,6,5,5,1,4,5,4,2,1,3,6,1,4,4,2,0,7,5,2,0,1,5$, $2,2,4,5,1,1,4]$

Generation's best community (list of genes):

NBN H2AFX RAD9A FANCD2 WRN BLM RAD50 KAT5 SUMO1 RPA2 FANCG MDC1 KAT2B E2F1 CREBBP ATR XRCC5 ING1 ABL1 RPA1 PIAS1 UBE2I MSH2 TP53BP1 MDM2 MRE11A MSH6 ESR1 RELA EP300 SUMO2 SP1 MCPH1 CHD3 XRCC6 PIAS4 RB1 CCND1 POU2F1 NCL PRKDC DCLRE1C SMARCA4 SMAD3 CHEK1 JUN NR3C1 RECQL5 HMGB1 HDAC2 DDX17 HDAC1 DAXX HIPK2 RBBP4 STAT5A PCNA UPF1 ETS1 KPNA2 PGR HIF1A TOP2A CDK1 NSD1

Number of genes inside the community: 65

*********************** Generation $295 * * * * * * * * * * * * * * * * * * * * * *$

Generation's average fitness: 0.54271337308023037

Generation's best fitness: 0.54944823962165

Generation's best individual:

$[5,2,2,5,7,7,7,7,3,2,3,1,4,7,2,3,1,4,5,5,1,7,0,2,4,6,4,7,6,7,1,3$, $0,1,5,4,5,6,0,5,2,0,2,0,5,2,0,2,5,0,4,1,2,5,2,7,6,6,0,4,7,7,0,7,0$, $0,2,6,1,3,4,7,1,0,6,0,1,4,4,2,7,1,6,2,6,0,2,2,1,3,4,4,0,0,3,6,3,1$, $7,3,3,1,0,5,1,2,4,0,3,0,0,6,1,2,3,6,5,1,4,1,1,5,4,5,1,5,7,5,3,1,4$, $4,4,0,1,5,0,4,5,5,7,1,1,0,1,2,3,5,2,0,5,7,3,4,4,7,2,1,7,3,2,3,5,6$, $4,0,2,3,2,5,4,0,6,2,0,6,1,1,2,6,4,0,3,0,4,4,3,6,5,1,4,3,1,0,7,0,4$, $0,5,4,0,3,4,5,1,2,2,4,3,1,3,6,3,6,4,6,2,6,5,2,5,7,6,4,7,1,3,7,0,1$, $7,6,4,2,5,2,0,2,1,5,3,6,4,2,4,6,7,4,7,4,6,0,4,5,4,0,7,5,6,3,7,7,1$, $3,2,0,7,7,0,1,1,5,5,7,1,4,0,6,3,7,6,1,1,7,3,7,6,7,3,0,6,7,1,1,5,1$, $0,3,7,6,4,6,7,4,5,0,0,3,3,1,6,0,3,1,4,2,7,4,5,4,1,2,0,1,3,1,4,3,6$, $4,0,5,6,2,2,2,2,4,1,3,3,5,1,5,0,5,4,4,3,0,6,6,5,0,2,3,3,4,2,1,2,0$, $2,5,0,4,2,3,5,3,3,1,4,2,0,2,2,5,4,7,1,3,7,2,7,6,7,6,0,5,3,2,3,2,7$, $7,7,5,5,0,2,5,4,1,2,6,6,6,2,0,5,2,7,2,5,3,3,6,6,6,4,4,5,2,2,7,7,6$, $4,4,5,5,3,0,2,3,4,3,7,3,7,2,4,1,6,6,2,1,2,2,0,1,7,1,1,2,4,6,5,2,1$, $3,2,6,3,7,3,7,5,7,0,2,4,2,0,1,4,7,0,2,1,3,6,1,5,4,2,0,7,3,2,7,1,6$, $2,2,4,5,1,7,5]$

Generation's best community (list of genes):

NBN H2AFX RAD9A FANCD2 WRN BLM RAD50 KAT5 SUMO1 RPA2 MDC1 KAT2B E2F1 CREBBP ATR XRCC5 ING1 ABL1 RPA1 CXXC5 PIAS1 UBE2I MSH2 TP53BP1 MDM2 MRE11A MSH6 ESR1 RELA EP300 SUMO2 SP1 MCPH1 
CHD3 XRCC6 PIAS4 RB1 CCND1 POU2F1 NCL PRKDC DCLRE1C SMARCA4 SMAD3 ERCC3 USP1 CHEK1 JUN NR3C1 RECQL5 HMGB1 HDAC2 DDX17 HDAC1 DAXX HIPK2 RBBP4 STAT5A PCNA ETS1 KPNA2 PGR HIF1A TOP2A CDK1

Number of genes inside the community: 65

********************** Generation 296 6*********************

Generation's average fitness: 0.54266199127150265

Generation's best fitness: 0.55520833333333335

Generation's best individual:

$[5,0,2,5,7,2,0,2,5,2,3,1,4,7,2,3,1,4,5,4,1,6,0,2,4,6,4,4,6,4,1,3$, $4,1,5,4,7,6,0,2,2,0,2,2,5,2,0,2,5,0,4,1,7,3,5,3,0,3,0,3,7,7,3,3,6$, $5,4,0,1,3,4,7,1,0,3,0,1,4,4,2,7,1,6,2,6,0,0,2,1,3,4,4,0,0,3,6,3,1$, $7,3,3,1,0,5,1,3,4,3,3,0,0,6,1,2,3,6,3,1,7,1,1,5,4,5,1,6,4,3,3,6,4$, $6,4,0,1,5,0,4,5,5,7,1,1,7,1,0,4,5,2,0,5,7,3,4,4,7,2,1,7,3,2,3,5,6$, $4,6,2,3,2,5,4,0,1,2,0,6,1,1,2,6,4,0,3,0,4,4,3,6,5,1,4,3,1,0,7,0,4$, $0,5,4,0,3,4,5,1,2,2,4,3,1,3,6,6,6,4,0,2,6,5,2,5,7,7,5,2,1,1,7,0,1$, $7,6,2,2,5,5,4,4,1,5,3,6,4,6,0,0,7,4,7,4,6,0,4,1,4,7,6,5,6,3,4,7,1$, $3,2,0,7,7,0,1,1,5,5,7,1,1,0,5,0,7,6,1,1,7,4,7,6,7,3,0,6,7,1,1,5,1$, $0,3,7,6,2,4,4,3,2,5,7,3,3,1,3,0,4,5,4,2,6,0,0,0,3,6,1,1,6,1,4,3,6$, $4,4,5,6,2,6,2,6,4,1,3,3,5,1,5,0,5,4,4,6,0,5,6,5,0,0,3,3,4,2,1,2,0$, $2,5,0,6,5,5,7,2,5,1,6,1,7,5,3,2,7,7,1,3,7,7,7,6,7,6,7,2,3,3,2,3,7$, $7,2,6,5,0,2,5,4,1,2,6,7,3,7,0,3,1,5,0,6,7,5,3,6,6,4,4,2,4,7,0,5,5$, $6,2,5,3,7,4,6,0,4,3,7,3,3,3,6,1,6,3,4,1,6,0,0,1,7,1,1,2,4,4,3,2,1$, $3,2,7,3,7,3,7,5,5,4,2,6,5,5,1,4,5,4,2,1,3,6,1,4,4,2,0,5,5,2,5,1,6$, $2,2,6,5,1,6,7]$

Generation's best community (list of genes):

NBN H2AFX RAD9A FANCD2 WRN BLM RAD50 KAT5 SUMO1 RPA2 MDC1 KAT2B E2F1 CREBBP ATR XRCC5 ING1 ABL1 RPA1 PIAS1 UBE2I MSH2 TP53BP1 MDM2 PSMB9 MRE11A MSH6 ESR1 RELA EP300 SUMO2 SP1 SAFB2 MCPH1 CHD3 RRM2 XRCC6 PIAS4 RB1 CCND1 UBE2U POU2F1 NCL PRKDC DCLRE1C SMARCA4 SMAD3 PPP2R2B CHEK1 JUN NR3C1 RECQL5 HMGB1 HDAC2 CBR3 DDX17 HDAC1 SRY DAXX HIPK2 RBBP4 STAT5A PCNA ETS1 KPNA2 PGR HIF1A TOP2A CDK1

Number of genes inside the community: 69

********************** Generation 297 \%*********************

Generation's average fitness: 0.54358081972197413 
Generation's best fitness: 0.55049921177088805

Generation's best individual:

$[5,0,2,5,7,2,2,2,3,2,3,1,4,7,2,3,1,4,5,5,1,6,0,2,4,6,4,7,1,4,1,3$, $4,1,5,4,7,5,0,5,2,0,2,0,5,2,0,2,5,0,4,1,2,3,5,3,0,3,0,4,7,7,0,7,6$, $5,2,6,1,4,3,2,1,3,6,5,1,4,4,2,7,1,6,2,0,0,2,2,1,3,4,4,0,4,3,6,3,1$, $7,3,3,1,0,5,1,3,4,3,6,5,6,2,1,4,4,6,5,1,7,1,1,0,7,3,1,6,4,4,6,6,4$, $0,4,0,1,5,0,4,5,5,7,1,1,4,1,2,3,5,2,0,5,7,3,4,4,7,2,1,7,3,2,3,5,6$, $4,0,2,3,2,5,4,0,6,2,0,6,1,1,2,5,4,0,3,0,4,4,3,6,6,1,4,3,1,0,7,0,4$, $0,5,4,0,7,4,5,1,2,2,4,3,1,3,5,3,6,4,6,6,2,0,5,4,4,2,4,7,1,6,7,0,1$, $7,5,2,6,7,5,0,2,1,4,3,6,4,6,0,0,7,4,7,4,6,0,4,5,4,7,7,2,6,3,4,7,1$, $3,2,0,7,7,0,1,1,5,5,7,1,4,0,6,3,7,6,1,1,7,3,7,4,7,3,0,6,7,1,1,5,1$, $0,3,7,6,4,0,7,2,0,2,7,3,2,1,3,7,6,5,4,2,3,5,7,0,3,6,5,1,7,1,4,3,6$, $4,4,5,6,0,2,2,2,3,1,3,3,5,1,5,0,5,4,4,3,0,5,6,5,0,0,3,5,4,2,1,2,0$, $2,5,2,6,6,0,4,7,5,1,3,6,7,5,5,4,7,7,1,3,7,2,7,6,7,6,7,2,3,3,2,3,0$, $0,2,1,5,0,3,3,4,1,2,0,6,3,0,2,1,3,3,0,6,7,3,6,6,0,7,3,2,4,2,0,5,7$, $4,4,5,5,7,4,6,0,4,3,7,3,3,3,6,1,6,6,2,1,6,0,7,1,7,1,1,2,4,2,3,2,1$, $3,2,2,7,4,3,7,5,7,4,2,7,2,4,1,4,5,4,2,1,3,6,1,4,6,2,4,7,5,2,5,1,4$, $6,6,7,4,1,7,5]$

Generation's best community (list of genes):

NBN H2AFX RAD9A TDP1 FANCD2 WRN BLM RAD50 KAT5 SUMO1 RPA2 MDC1 KAT2B E2F1 CREBBP ATR XRCC5 ING1 ABL1 RPA1 PIAS1 UBE2I MSH2 TP53BP1 MDM2 MRE11A MSH6 ESR1 RELA EP300 SUMO2 SP1 MCPH1 CHD3 XRCC6 PIAS4 RB1 CCND1 POU2F1 NCL PRKDC DCLRE1C SMARCA4 SMAD3 CHEK1 JUN NR3C1 RECQL5 HMGB1 HDAC2 DDX17 AURKA HDAC1 NUCB1 DAXX HIPK2 RBBP4 STAT5A PCNA ETS1 KPNA2 PGR HIF1A TOP2A CDK1

Number of genes inside the community: 65

********************* Generation $298 * * * * * * * * * * * * * * * * * * * *$

Generation's average fitness: 0.5429945322133931

Generation's best fitness: 0.55170603674540685

Generation's best individual:

$[5,0,2,5,6,2,2,2,3,2,3,1,4,7,2,3,1,4,5,5,1,6,0,4,4,6,4,7,6,4,1,3$, $4,1,5,4,7,2,3,2,2,0,2,0,5,2,0,2,5,0,4,1,2,3,5,3,0,5,0,3,7,5,6,7,0$, $7,0,0,1,4,3,2,1,3,3,6,1,4,4,2,7,1,6,2,5,6,0,6,1,3,4,4,0,0,3,6,3,1$, $7,3,3,1,0,5,1,3,4,3,3,0,0,6,1,2,3,6,4,1,7,1,1,5,5,6,1,6,4,3,3,6,6$, $4,4,0,1,5,0,4,5,5,7,1,1,0,1,2,3,4,2,0,5,7,6,0,6,7,2,1,7,3,2,2,5,6$, 
4, 0, 2, 3, 2, 5, 4, 0, 6, 2, 0,6, 1, 1, 3, 6, 4, 0, 3, 0, 4, 4, 3, 6, 5, 1, 4, 3, 1, 0, 7, 0, 4, $0,5,4,0,3,4,5,1,5,2,4,3,1,3,6,3,6,4,2,7,4,5,2,6,4,6,4,4,1,3,2,0,1$, $7,5,2,2,5,2,0,2,1,5,3,6,4,6,0,0,7,4,7,7,6,0,4,5,4,7,6,5,6,3,7,3,1$, $3,0,0,7,7,0,1,1,5,5,7,1,4,0,6,3,7,6,1,1,7,3,7,6,7,3,0,6,7,1,1,5,1$, $0,3,7,6,0,0,7,2,0,0,7,3,2,1,6,0,3,5,4,2,7,0,6,0,3,5,5,1,7,1,4,3,6$, $4,0,5,6,0,7,3,6,4,1,3,3,5,1,5,0,5,4,4,3,0,5,6,5,0,0,3,3,4,2,1,3,0$, $4,5,0,4,2,3,5,3,3,1,6,6,7,2,2,5,7,7,1,3,7,7,7,6,7,6,7,3,1,2,3,2,7$, $7,2,6,5,0,2,5,2,1,2,6,6,3,0,0,3,3,5,0,6,0,5,3,6,6,4,2,5,4,2,0,5,6$, $0,4,5,5,7,4,6,0,4,3,7,3,3,3,6,1,2,7,4,1,6,0,0,1,7,1,1,2,0,6,1,2,1$, $3,2,7,3,7,3,7,5,5,4,3,7,4,0,1,4,5,4,2,1,3,6,1,4,4,2,0,7,5,2,5,1,6$, $2,2,4,5,1,7,3]$

Generation's best community (list of genes):

NBN H2AFX RAD9A FANCD2 WRN BLM RAD50 KAT5 SUMO1 RPA2 MDC1 KAT2B E2F1 CREBBP ATR XRCC5 ING1 ABL1 RPA1 PIAS1 UBE2I MSH2 TP53BP1 MDM2 MRE11A MSH6 ESR1 RELA EP300 SUMO2 SP1 MCPH1 CHD3 XRCC6 PIAS4 RB1 CCND1 POU2F1 NCL PRKDC DCLRE1C SMARCA4 SMAD3 CHEK1 JUN NR3C1 RECQL5 HMGB1 HDAC2 DDX17 CDC25C HDAC1 DAXX HIPK2 RBBP4 STAT5A PCNA UPF1 ETS1 KPNA2 PGR HIF1A TOP2A CDK1

Number of genes inside the community: 64

**********************Generation 299 **********************

Generation's average fitness: 0.5431369249076504

Generation's best fitness: 0.5535882661079099

Generation's best individual:

$[0,0,2,5,7,2,2,2,3,2,3,1,4,7,2,3,1,4,5,5,1,6,0,4,4,6,4,7,6,4,1,2$, $4,1,5,4,5,6,0,5,2,0,2,0,5,2,0,2,5,6,4,1,0,3,5,2,5,5,0,4,7,3,0,7,0$, $0,2,6,1,3,4,7,1,0,6,0,1,4,4,2,7,1,6,2,6,5,2,6,1,3,4,4,0,0,3,4,7,1$, $7,3,3,1,0,5,1,3,4,3,3,0,6,6,1,2,3,6,5,1,4,1,1,0,4,5,1,5,7,4,6,6,4$, $6,4,0,1,6,0,4,5,5,7,1,1,0,1,2,3,5,4,4,4,6,3,4,4,7,2,1,7,3,2,3,5,6$, $4,0,2,3,2,5,4,0,6,2,0,6,1,1,2,6,4,0,3,0,4,4,3,6,5,1,4,3,1,0,7,0,4$, $0,5,4,0,2,4,5,1,2,2,4,3,1,3,4,3,6,4,6,2,6,5,2,5,7,7,5,2,1,3,7,0,1$, $7,6,2,6,7,5,0,2,1,5,6,6,4,6,0,0,7,4,7,4,6,0,4,1,3,4,7,5,6,3,5,7,1$, $3,2,0,7,7,0,1,1,5,5,7,1,4,0,0,3,7,6,1,1,7,3,7,6,7,3,0,6,7,1,1,5,1$, $0,3,7,6,0,0,7,4,2,2,7,3,2,1,6,0,5,5,4,2,7,0,0,0,3,6,5,1,7,1,4,3,6$, $5,4,5,6,2,2,3,2,1,1,6,3,5,1,5,0,5,4,4,3,4,5,6,5,0,0,3,5,5,2,1,2,5$, $7,2,0,4,2,3,5,3,3,1,6,6,7,5,5,4,7,7,1,7,6,6,2,2,3,3,7,2,3,5,6,6,2$, $0,0,5,5,0,2,5,4,1,6,6,7,6,7,0,3,3,5,0,6,7,5,6,4,3,4,3,5,2,2,7,7,6$, 
4, 4, 5, 5, 7, 4, 6, 0, 3, 3, 7, 3, 3, 3, 6, 1, 2, 7, 4, 1, 6, 0, 1, 1, 7, 1, 1, 6, 4, 4, 3, 2, 1, $3,2,7,3,7,3,7,5,5,4,2,7,4,0,1,4,5,4,2,1,3,5,1,4,4,2,0,7,5,6,5,1,6$, $2,2,4,5,1,7,4]$

Generation's best community (list of genes):

NBN H2AFX RAD9A FANCD2 WRN BLM RAD50 KAT5 SUMO1 RPA2 MDC1 KAT2B E2F1 CREBBP ATR XRCC5 ING1 ABL1 RPA1 PIAS1 UBE2I MSH2 TP53BP1 MDM2 MRE11A MSH6 ESR1 RELA EP300 SUMO2 SP1 MCPH1 CHD3 RRM2 XRCC6 PIAS4 RB1 CCND1 POU2F1 NCL PRKDC DCLRE1C SMARCA4 SMAD3 CHEK1 JUN TAOK3 NR3C1 RECQL5 HMGB1 HDAC2 DDX17 HDAC1 DAXX HIPK2 MTA1 RBBP4 STAT5A PCNA ETS1 KPNA2 PGR HIF1A TOP2A CDK1

Number of genes inside the community: 65

**********************Generation $300 * * * * * * * * * * * * * * * * * * * * *$

Generation's average fitness: 0.54361712080152834

Generation's best fitness: 0.5512374934175882

Generation's best individual: $[5,0,2,2,2,5,0,4,3,2,3,1,4,7,2,3,1,4,5,5$, $1,0,0,2,4,6,4,4,6,4,1,3,4,1,5,4,7,6,0,5,2,4,2,0,5,2,0,2,5,0,4,1,2$, $3,5,3,6,5,0,7,7,7,0,7,0,5,3,0,1,4,4,7,1,0,6,0,1,4,4,2,7,1,6,2,6,0$, $2,6,1,3,4,4,0,0,3,4,7,1,7,3,3,1,0,5,1,2,4,0,6,0,0,6,1,2,3,6,5,1,4$, $1,1,5,5,6,1,6,4,3,3,1,7,4,4,0,1,5,0,4,5,5,7,1,1,0,1,2,3,5,2,0,5,4$, $3,4,4,7,2,1,7,3,2,3,5,6,4,0,2,3,2,5,4,0,6,2,0,6,1,1,2,6,4,0,3,0,4$, $4,3,6,5,1,4,3,1,0,7,0,4,0,5,4,0,3,4,5,1,2,2,4,3,1,3,6,3,6,4,6,2,6$, $5,2,4,4,2,4,7,1,3,7,0,1,7,6,2,6,7,5,0,2,1,5,3,6,4,6,0,0,7,4,7,7,4$, $4,5,5,4,7,6,5,6,3,4,7,1,3,3,0,7,7,0,1,1,5,5,7,1,4,0,5,0,7,6,1,1,7$, $3,7,6,7,3,0,6,7,1,1,5,1,0,3,7,6,0,0,7,4,5,0,0,3,3,1,3,0,4,5,4,2,7$, $0,6,0,3,6,5,1,7,1,4,3,6,5,4,5,6,2,6,2,2,4,1,3,3,5,1,5,0,5,4,1,3,0$, $5,6,5,0,0,3,5,5,6,1,7,0,4,5,0,4,2,3,5,3,3,1,6,6,3,5,5,4,7,7,1,3,7$, $7,7,6,7,6,6,2,3,2,3,2,7,7,3,3,5,0,2,5,6,1,6,6,6,3,0,2,4,6,3,5,6,7$, $5,7,7,3,4,4,2,2,7,5,5,6,0,6,5,5,7,4,6,0,4,3,7,3,3,2,6,1,0,3,4,1,6$, $0,0,1,7,1,1,2,4,6,5,2,1,3,2,6,3,7,3,4,2,3,5,2,6,5,5,1,4,5,4,2,1,3$, $6,1,4,4,2,0,7,5,2,5,1,6,2,2,4,5,1,7,0]$

Generation's best community (list of genes):

NBN H2AFX RAD9A FANCD2 WRN BLM RAD50 KAT5 SUMO1 RPA2 MDC1 KAT2B E2F1 CREBBP ATR XRCC5 ING1 ABL1 RPA1 CXXC5 PIAS1 UBE2I MSH2 TP53BP1 MDM2 MRE11A MSH6 ESR1 RELA EP300 SUMO2 SP1 MCPH1 CHD3 XRCC6 PIAS4 RB1 CCND1 POU2F1 NCL PRKDC DCLRE1C SMARCA4 
SMAD3 CHEK1 JUN NR3C1 RECQL5 BUB1B HMGB1 HDAC2 DDX17 HDAC1 DAXX HIPK2 RBBP4 STAT5A PCNA ETS1 KPNA2 PGR HIF1A TOP2A CDK1 Number of genes inside the community: 64 


\section{Appendix B}

\section{Samples of generated candidate genes ranking}

\section{B.1 A candidate genes ranking for Breast Cancer}

This is a candidate genes ranking generated by our method for the Breast Cancer experiment (Section 8.1) while the known disease gene BRCA1 is taken out in leaveone-out cross validation. The scores assigned to the genes are in front of the genes' names in the ranking. Here, we show the top 100 genes in the ranking.

0) BRCA1: 369454.94228473795

1) MDM2: 365856.40243225696

2) H2AFX: 365237.2320897151

3) SUMO1: 364293.4178539702

4) HDAC1: 362886.60125484853

5) SP1: 362874.9776684882

6) ESR1: 361630.4298796606

7) KAT5: 361257.9720975167

8) NR3C1: 360883.2030843063

9) RELA: 359544.9900832348

10) SMAD3: 358477.115271801

11) MDM4: 355867.4739784314

12) PRKDC: 355018.0532636549

13) ABL1: 353907.1203097202

14) JUN: 353339.7953658223 
15) E2F1: 352549.3955796374

16) CREBBP: 349253.1080401208

17) POU2F1: 348262.4847448964

18) EP300: 347403.97396584525

19) UBE2I: 346219.77838602086

20) ETS1: 345556.8781321825

21) DAXX: 344091.03157358

22) PIAS4: 343545.1564074639

23) YWHAH: 342920.3874388374

24) HIF1A: 339962.5300479148

25) MDC1: 339732.12818675727

26) AKT1: 339502.7485254788

27) TOP2A: 339092.5080355513

28) PIAS1: 336002.2311787074

29) RB1: 336001.3209315408

30) HSP90AA1: 335861.6493675062

31) XRCC6: 335697.49964056996

32) HIPK2: 334935.9014436146

33) TP53BP1: 333485.33286573633

34) KAT2B: 333377.67930907797

35) CCND1: 332936.77745437383

36) HDAC2: 330880.3869432769

37) PLAGL1: 329302.65018068627

38) FOXO3: 326577.7248249773

39) NBN: 324070.81259756564

40) CHUK: 322317.60499559686

41) SMARCA4: 310588.1376046659

42) SIRT1: 308388.9244616711

43) CHEK1: 305480.56542420975

44) HMGB1: 301342.0173945646

45) XRCC5: 299469.47085596377

46) CTBP1: 272690.1749045091

47) WRN: 266131.3596648345

48) RBBP4: 250985.69499962227

49) DDX5: 224903.51704771272

50) NCL: 126840.421261955 
51) FANCD2: 101600.45916398258

52) CHD3: 96326.29414206218

53) CDK1: 90571.79220528794

54) MRE11A: 54911.26557501975

55) WHSC1: 36001.443298332735

56) ATR: 31125.17269410985

57) MMS19: 31094.612380380306

58) CSNK2A2: 30034.350954123107

59) HAP1: 23725.391412693112

60) MED1: 20633.865453793773

61) E4F1: 19860.279556357083

62) MTA3: 19542.109175809474

63) IKBKB: 19376.1666742751

64) BRE: 19118.485820483253

65) KAT7: 18502.845784892746

66) NR0B2: 18449.536790455604

67) BRCC3: 16065.606781765428

68) ESRRA: 15971.35206842881

69) CNTN2: 15694.080402161117

70) PLK1: 14493.024891180312

71) VDR: 13364.963237045538

72) RAD9A: 13060.451853699244

73) PTK2: 12771.512829408597

74) CASP3: 12609.978851736856

75) MED17: 12448.436116885665

76) UBE3A: 11680.659205093381

77) FLNA: 11487.892863898282

78) MSH2: 11381.53438558635

79) RCHY1: 11244.836174057624

80) KPNA2: 11046.0458324487

81) BLM: 10819.456320267906

82) PPP2R2B: 10770.971832395737

83) NRIP1: 10732.09559316361

84) HTT: 10272.060948911978

85) RPA1: 10268.327869469502

86) CTDSP2: 9890.459988378514 
APPENDIX B. SAMPLES OF GENERATED CANDIDATE GENES RANKING87

87) GPR25: 9260.253646191584

88) PIK3R1: 9110.426947089396

89) IKBKG: 9045.73201802221

90) IFI16: 8931.097621089097

91) AATF: 8353.544101357822

92) ZMIZ1: 8129.712555231577

93) SRA1: 7932.790750478763

94) CARM1: 7907.00907829512

95) MAST1: 7747.4187911663

96) PPARG: 7674.949592467252

97) NCOA2: 7667.309422367735

98) FOXA1: 7556.888859662708

99) ERCC2: 7208.141947439502

100) ING1: 7107.157733510438

\section{B.2 A candidate genes ranking for Parkinson's dis- ease}

This is a candidate genes ranking generated by our method for the Parkinson's disease experiment (Section 8.1) while all the known disease genes provided in Section 8.3.1 are in the known disease gene set. The scores assigned to the genes are in front of the genes' names in the ranking. Here, we show the top 100 genes in the ranking.

0) UBC: 4144932.8105707495

1) TAT: 4128398.286184617

2) SMAD3: 4117814.8295134627

3) TP53: 4114948.6209134124

4) EP300: 4114724.3239404806

5) SRC: 4113482.1294503445

6) EGFR: 4112064.4392586253

7) CREBBP: 4111022.0977524514

8) SP1: 4105014.840084765

9) ABL1: 4104748.7384657217

10) JUN: 4103186.5143881436

11) RELA: 4102431.8847347354 
12) HSP90AA1: 4102178.605043044

13) AKT1: 4095084.991584094

14) STAT3: 4093262.201525781

15) HDAC1: 4092874.878398885

16) MYOD1: 4090765.7342951

17) LYN: 4089375.436431014

18) ESR1: 4088046.2204013253

19) GRB2: 4086223.208137686

20) HDAC3: 4082942.1262650196

21) AR: 4081947.020808688

22) STAT5A: 4077919.927194697

23) ESR2: 4076391.7036378426

24) HSPA8: 4074203.9452217068

25) BAD: 4066635.5092346147

26) RB1: 4065199.3373429505

27) SMARCA4: 4065085.3752701147

28) MDM2: 4063539.3668733574

29) SYK: 4057721.61894727

30) MYC: 4056827.3936953745

31) HIF1A: 4056087.9086310626

32) CDK1: 4056026.209885783

33) CHUK: 4053287.7350594043

34) YWHAH: 4050678.335009849

35) LCK: 4043770.109977208

36) EIF2AK2: 4043697.3419758556

37) HSPA4: 4042615.6230233596

38) KLF6: 4042590.7282105037

39) SMAD2: 4037764.3273650063

40) PRKCA: 4037578.2941328348

41) BRCA1: 4035108.802434509

42) MAPK1: 4034171.2175798165

43) NCOR2: 4033360.6318513053

44) NFKBIA: 4033242.3780480307

45) CSNK2A1: 4033131.8388114134

46) NR3C1: 4032370.851070569

47) ETS1: 4024623.570533809 
48) CEBPA: 4024610.5106870024

49) CSK: 4023375.9459752073

50) EPHA1: 4020423.8736465573

51) CASP8: 4019355.1507314397

52) PRKCZ: 4018673.224499437

53) SHC1: 4016726.7234874023

54) EGR1: 4016269.0185094913

55) STUB1: 4015350.104557534

56) SIN3A: 4014752.863356188

57) STAT5B: 4008235.459306945

58) CRKL: 4004772.156934122

59) PTPN1: 4003784.895714837

60) SMAD4: 4003559.2876090994

61) PRKCD: 4003477.4780933433

62) FANCG: 4002672.348805875

63) PTK2: 4000161.655493187

64) PXN: 3995887.0923527624

65) CDKN1B: 3995754.0823792685

66) PIK3R1: 3992324.183723157

67) MAPK3: 3990811.4157836037

68) MAPK8: 3984679.7284387522

69) UBE3A: 3983827.2541076583

70) SIRT1: 3982848.98963996

71) CAV1: 3980913.693707568

72) RAF1: 3979357.6771421265

73) CCND1: 3977433.727264135

74) CTNNB1: 3977162.5125806155

75) PTK2B: 3965741.111429293

76) IGF1R: 3965466.2120283684

77) NFATC2: 3965301.584928153

78) PIAS3: 3962397.4256757107

79) BAG1: 3961988.3006393053

80) JAK2: 3954214.122580029

81) RPS6KA5: 3953046.4416621784

82) CDKN1A: 3952485.009627919

83) KRT18: 3946100.961114532 
84) MAP3K5: 3945876.2108147405

85) TP73: 3944474.9916920615

86) FYN: 3943866.974338102

87) BCL2: 3941653.613609305

88) PPP1CA: 3938345.2998506497

89) YWHAZ: 3936490.3977781427

90) FOXO1: 3935436.835571163

91) HDAC2: 3929580.74902719

92) FOXO3: 3922362.575982028

93) PRKCE: 3919600.5134588154

94) YWHAE: 3919510.434878021

95) CASP3: 3909988.523816787

96) MAP3K7: 3908175.90877334

97) RPS6KB1: 3907969.56665488

98) CCNE1: 3907285.010561756

99) FOXO4: 3904962.5682972665

100) GSK3B: 3903640.0271610236 


\section{Appendix C}

\section{Sample disease genes communities}

In this appendix, we provide the disease gene communities with the highest modularity (Q - see equation6.1)) given by our method for the experiments explained in Chapter 8.

\section{C.1 Breast Cancer}

The best community produced by our method for Breast Cancer experiment (Section 8.1) contained the following 97 genes (not in any order of ranking): USP9Y, MAP1LC3B, CDKN1B, SEPT4, HSPA8, RNF41, MARK4, AXIN1, CDC42SE1, NDUFS4, CYP19A1, LIMK1, SLC25A1, GNAZ, THY1, SULT1A2, RBCK1, PCSK9, GRK6, CDKN2A, MTF1, SIGLEC10, SLC25A12, PPP1R2, PPP2CA, ITGB1BP1, TMEM160, ESR1, ADRBK1, NUMA1, B2M, TSC1, LRP1B, DRD1, KARS, ANXA6, S1PR1, OSGEP, CYTH2, DYNLL2, SLC29A2, PPP1CA, RARS, ARHGAP1, SLX4, SDC3, GJB3, DVL1, CDK6, PTPRO, ETHE1, GPS1, CDKN2AIP, AIMP1, CNTNAP4, EIF2C4, ACTG1, L1CAM, RBX1, IQGAP1, KIF20B, FOXO3, MAPK8IP3, ITGB4, TFB2M, LARP4B, ANXA1, STOML2, CHORDC1, RPE65, FOXM1, SMAD2, SLC8A1, TJAP1, EMD, ALDOB, ZNF91, NKD2, XBP1, TUBB4B, PHOX2A, KPNA1, EID3, HSPB1, MDN1, VAV2, RIC8A, UBE2A, GNA12, EREG, CLTB, ABI1, EID1, CDC40, PTRF, TTN, MKKS,

\section{C.2 Parkinson's disease}

The best community produced by our method for Parkinson's disease experiment (Section 8.3) contained the following 100 genes (not in any order of ranking): NTRK2, 
SCARB2, NTF4, NTRK1, ALK, UBASH3A, SNX17, SH2B1, FRS2, PTPN1, FRS3, EGFR, PLCG1, FYN, NCK2, MATK, CSK, FGR, ARHGAP32, CTNND1, CAV2, MST1R, SHC1, DOCK1, GRB2, PLD2, INSR, ERBB3, IRS1, ABL1, NCK1, GHR, FGFR1, PAX7, ITGB3, JAK2, PTPRB, PIK3R1, PTPRJ, PTPN12, IL6ST, RET, FLT1, PTK2B, PDGFRB, SYK, CBL, PTPRO, SRC, AXL, BCAR1, LYN, LTK, STAT3, MET, CRK, BMX, ,PTK2, SNX3, PTPRC, PDGFRA, GAB1, KDR, IGF1R, SOS1, PTPN11, PXN, LCK, DDR1, ERBB2, IRS2, KIT, SYNJ2, DLGAP2, IL4R, RASA1, CD2AP, VAV3, SOCS7, JAK3, SOCS1, CEACAM1, VAV1, EFS, DLGAP1, CXCR4, DOK1, EPOR, CRKL, AKAP2, SOS2, PTPN6, STAT5A, GRB7, DOCK3, ABI1, BCR, STAT5B, PDIA2, PIK3C2B, PIK3R2. 